\title{
Frequency Domain Equalization for High Data Rate Multipath Channels
}

by

Pawel Adam Dmochowski

\author{
$A$ thesis submitted to the \\ Department of Electrical and Computer Engineering \\ in conformity with the requirements \\ for the degree of Master of Science (Engineering)
}

Queen s Lniversity

Kingston. Ontario. Canada

March 2001

Copyright (C) Pawel Adam Dmochowski. 2001 
National Library

of Canada

Acquisitions and Bibliographic Services

395 Wellington Street

Otlawa ON KIA ON4

Canada
Bibliothèque nationale

du Canada

Acquisitions et

services bibliographiques

395. nue Wellington

Ottawa ON KIA ONA

Canada
The author has granted a nonexclusive licence allowing the National Library of Canada to reproduce, loan, distribute or sell copies of this thesis in microform, paper or electronic formats.

The author retains ownership of the copyright in this thesis. Neither the thesis nor substantial extracts from it may be printed or otherwise reproduced without the author's permission.
L'auteur a accordé une licence non exclusive permettant à la Bibliothèque nationale du Canada de reproduire, prêter, distribuer ou vendre des copies de cette thèse sous la forme de microfiche/film, de reproduction sur papier ou sur format électronique.

L'auteur conserve la propriété du droit d'auteur qui protège cette thèse. $\mathrm{Ni}$ la thèse ni des extraits substantiels de celle-ci ne doivent être imprimés ou autrement reproduits sans son autorisation. 


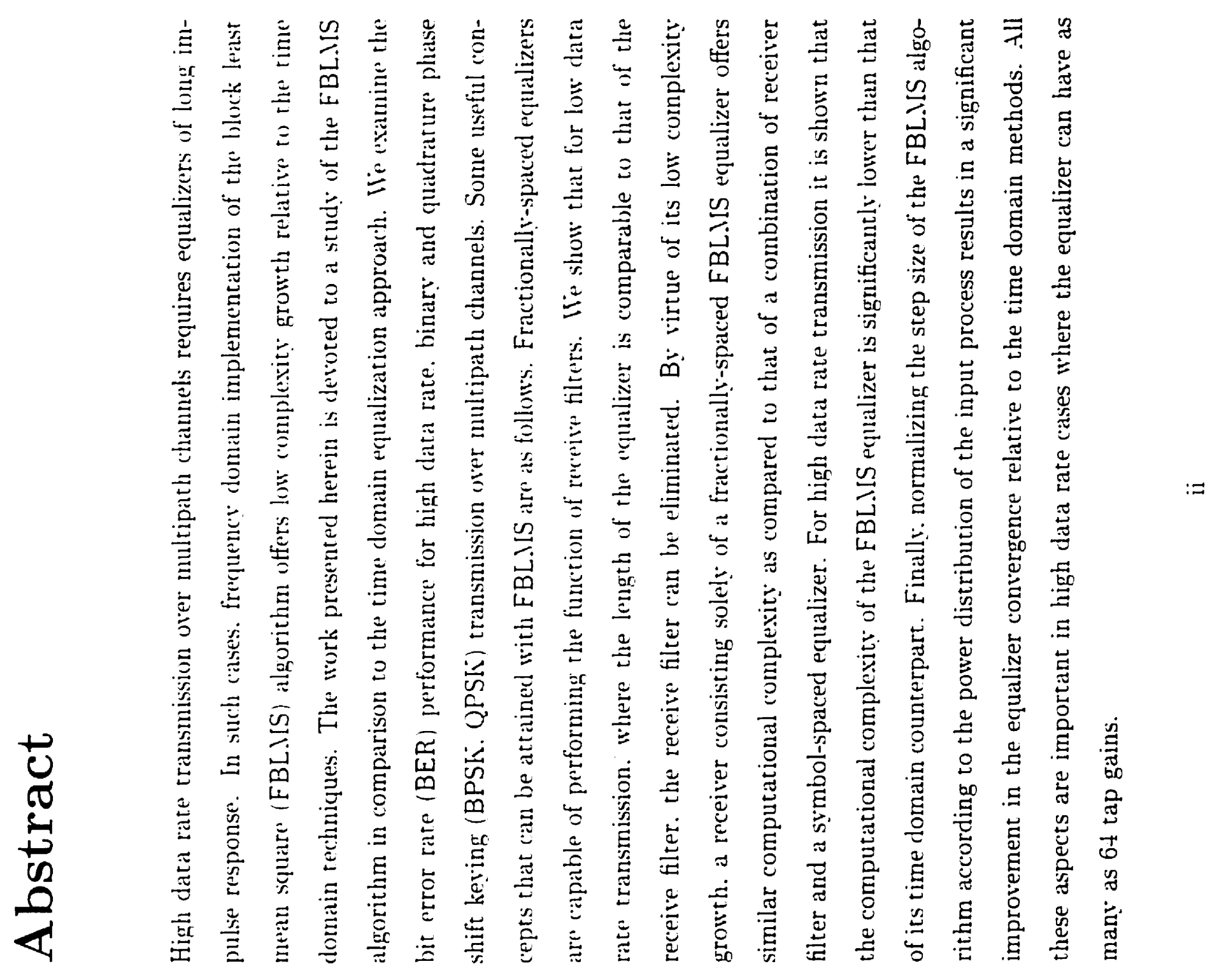


To my loving fomuly 


\section{Acknowledgements}

I would like to take this opportunity to extend my gratitude to all those who have made this work possible.

First and foremost. I wish to thank my supervisor. Prof. Peter J. I[cLane. whose guidance. support and never-ending patience throughout this thesis I greatly appreriate. I am grateful to the Natural Sciences and Engineering Research Council of Canada for their generous financial support.

I wonld also like to acknowledge the help [ have received from Prof. Saeed Giazor cluring the course of this work.

I thank all the faculty and staff of the Department of Electrical and Computer Engineering for their dedication and hard work. Together with my fellow students they have made the past couple of years truly fun and enjoyable.

Finalls: thank you. Nicole. for your constant support and all the reminders that I would "get there". Thanks to rour encouragement I can now say that you were right. 


\section{Table of Contents}

List of Tables viii

List of Figures $\quad$ ix

List of Abbreviations $\quad$ xi

List of Symbols $\quad$ xiii

1 Introduction 1

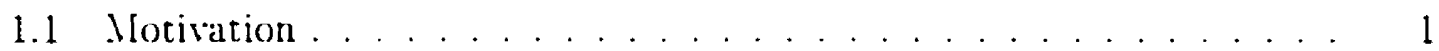

1.2 Literature Review . . . . . . . . . . . . . . . . . 2

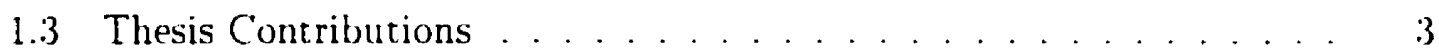

1.4 Thesis Outline . . . . . . . . . . . . . . . . . . j

2 Theoretical Background $\quad 6$

2.1 Introduction . . . . . . . . . . . . . . . . . 6

$2.2 \quad$ Wiener Filters . . . . . . . . . . . . . .

2.3 The L.MS Algorithm . . . . . . . . . . . . . . . . 10

2.3.1 Computational Complexity of the L.MS Algorithm . . . . . . 12

2.3.2 Convergence Properties of the L.IS Algorithm . . . . . . . 12

2.4 Transform Domain Equalization . . . . . . . . . . . . . . 15 
2.4.1 Computational Complexity of the TDL.IS Algorithm . . . 19

2.4 .2 Convergence Properties of the TDL.IS Algorithm . . . . . I9

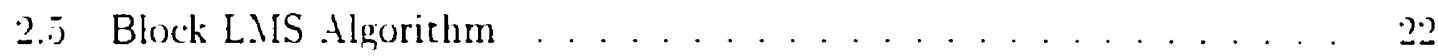

2.5.1 Convergence Properties of the BLAS Algorithm . . . . . . 2.3

2.5.2 Computational Complexity of the BL.IS Algorithm . . . . 2. 2t

2.6 Fast Block Least Mean Square Equalizors . . . . . . . . . . 2. 24

2.6 .1 Linear and Circular Convolution . . . . . . . . . . .

$2.6 .2 \quad$ Fast Block L.IS Algorithm . . . . . . . . . . . . . . . 28

2.6.3 Computarional Complexity of the FBL.IS algorithm $\ldots \ldots$. . .5.

3 Performance over Low Rate Channels $\quad 37$

3.1 Introduction . . . . . . . . . . . . . . . . . . . . . . 37

3.2 Communication Ststem Mociel . . . . . . . . . . . . . . 37

3.2 .1 Signaling Methods . . . . . . . . . . . . . . . . 39

$3.2 .2 \quad$ Pulse Shaping . . . . . . . . . . . . . . . . . 40

3.2 .3 Channel ........................... 41

3.2 .4 Equalizer . . . . . . . . . . . . . . . . . 41

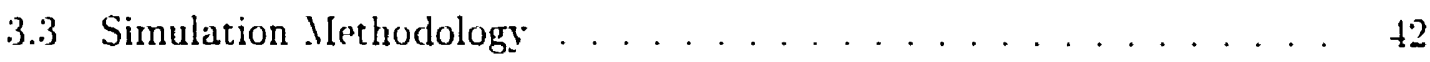

3.4 Bit Error Rate Performance . . . . . . . . . . . . . . . . 43

3.4.1 BER Sinulation Method . . . . . . . . . . . . . 43

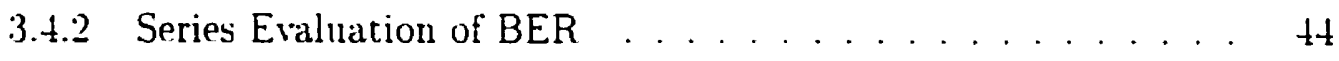

3.4 .3 BER Results . . . . . . . . . . . . . . . . 45

3.5 Convergence Properties . . . . . . . . . . . . . . . ti

3.6 Computational Complexity . . . . . . . . . . . . . . . . 53

4 Performance over High Rate Channels $\quad 57$ 
4.1 Introduction . . . . . . . . . . . . . . . . . . . . .

4.2 Bit Error Rate Performance . . . . . . . . . . . . . 64

4.2.1 BER Performance as a Function of S.TR . . . . . . . . 64

4.2.2 BER Performance ats a Function of Data Rate ........ il

4.2 .3 Channel Distortion Analysis .............. il

4.3 Convergence Properties . . . . . . . . . . . . . . . . . . . .

4.3 .1 Equalizer Learning Curres . . . . . . . . . . . . . ד

4.3.2 Correlation Matrix Eigenvalue Analysis . . . . . . . . . . $\$ 3$

t.4 Computational Complexity . . . . . . . . . . . . . . 85

5 Conclusion $\quad 88$

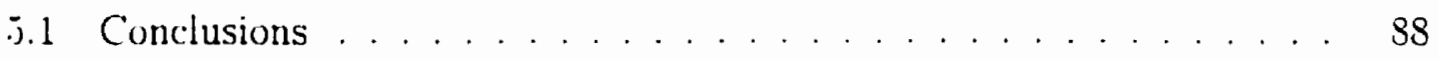

э.2 Suggestions for Further Research . . . . . . . . . . . . . . 90)

$\begin{array}{lr}\text { Bibliography } & 92\end{array}$

$\begin{array}{lr}\text { Vita } & 97\end{array}$ 


\section{List of Tables}

3.1 Complexity of the L.XS. FBL.IS and NFBL.MS Algorithms . . . . . jt

3.2 Complexity of a $T / 8$-spaced Receiver and a $T$-spaced Equalizer . . . jis

3.3 Complexity of a $T / 8$-spaced Equalizer . . . . . . . . . . . jij

4.1 High Rate Chamnel Eigentalue Spreat Comparison . . . . . . . . 8.3

4.2 Equalizer Complexity for High Rate Channels (BPSK) . . . . . . 86

t.3 Equalizer Complexity for High Rate Channels (QPSK) . . . . . . . 86 


\section{List of Figures}

$2.1 \quad+$ Transversal Filter $\ldots \ldots \ldots \ldots \ldots \ldots \ldots$

2.2 Transform Domain Adaptive Filter $\ldots \ldots \ldots \ldots$

2.3 ()wrlap-Add Method of Linear Convolution $\ldots \ldots \ldots \ldots$

2.4 () verlap-Save Method of Linear Convolution . . . . . . . . 30

2.5 FBL.IS Algorithm Ling Overlap-Sate Sectioning . . . . . . . . 32

3.1 Communication System . Iodel . . . . . . . . . . . . . . 38

3.2 Channel A BER Results $\ldots \ldots \ldots \ldots \ldots \ldots$

3.3 Channel B BER Results . . . . . . . . . . . . . ts

3.4 Channel $A$ Equalizer Learning Curres . . . . . . . . . . . . 50

3.j Channel B Equalizer Learning Cirres . . . . . . . . . . . . 51

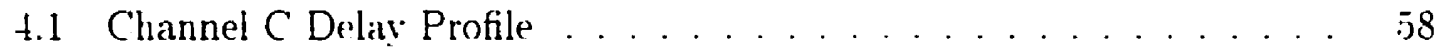

4.2 Channel D Delay Profile $\ldots \ldots \ldots \ldots \ldots \ldots$

4.3 Channel C (Case 1) Amplitude Spectrum . . . . . . . . . . 60

4.4 Channel C (Case 2) Amplitude Spectrum . . . . . . . . . . . 61

4.5 Channel D Amplitude Spectrum . . . . . . . . . . . . . . . 62

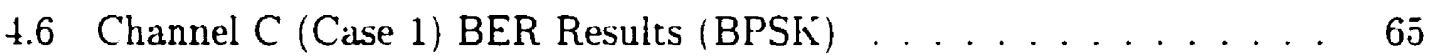

4.7 Channel C (Case 1) BER Results (QPSK) . . . . . . . . 66

4.8 Channel C (Case 2) BER Results (BPSK) . . . . . . . . 6 6 i 
4.9 Channel $\mathrm{C}$ (Case 2) BER Results (QPSK) ........... 68

4.10 Channel D BER Results (BPSK) . . . . . . . . . . . . . 69

4.11 Channel D BER Results (QPShi . . . . . . . . . . . त)

4.12 High Rate Channel BER as a Function of Datal Rate (BPSK) . . . . i2

4.13 High Rate Channel BER as a Function of Data Rate (QPSK) . . . . T3

4.14 High Rate Channel Input SIR . . . . . . . . . . . . i.j

4.15 High Rate Chatnel Input S.IR . . . . . . . . . . . . 76

4.16 High Rate Channel Output S.IIR (BPSK) . . . . . . . . T

4.1i High Rate Channel Output S.YR (QPSK) . . . . . . . . is

4.1S Channel C Equalizer Learning Curres (BPSK) . . . . . . . . 79

4.19 Channel D Equalizer Learning Curves (BPSK) . . . . . . . . . . so

4.2) Channel C Equalizer Learning Curves ((QPSK) . . . . . . . . . 81

4.21 Channel D Equalizer Learning Curres (QPSK) . . . . . . . . 82 


\section{List of Abbreviations}

\begin{tabular}{|c|c|}
\hline$A H^{\circ} C^{\circ}$ & Additive IThite Gaussian Noise \\
\hline BER & Bit Error Rate \\
\hline BL.MS & Block Least Mean Square \\
\hline BPSK & Binary Phase Shift Kerying \\
\hline$D C T$ & Discrete Cosine Transfurm \\
\hline DFT & Discrete Fourier Transform \\
\hline FBL.IS & Fast Block Least Mean Square \\
\hline FFT & Fast Fourier Transform \\
\hline ISI & Intersimbol Interference \\
\hline KLT & Karhunen-Loéve Transform \\
\hline L.ISS & Least Mean Square \\
\hline .IMISE & Minimum Mean Squared Error \\
\hline .ISE & . Mean Squared Error \\
\hline NFBLAIS & . Vurmalized Fast Block Least Mean Square \\
\hline QPSK & Quadrature Phase Shift Kering \\
\hline $\mathrm{RC}$ & Raised Cosine \\
\hline RLS & Recursive Least Square \\
\hline R.MS & Root . Mean Square \\
\hline $\mathrm{RRC}$ & Ruot Raised Cosine \\
\hline SIR & Signal-to-Interference Ratio \\
\hline
\end{tabular}


S.IR

S.IIR

TD.AF

TDM.A
Signal-to-.loise Ratio

Signal-to-.loise-and-Interference Ratio

Transform Domain Adaptive Filtering

Time Division Multiple Acress 


\section{List of Symbols}

\begin{tabular}{|c|c|}
\hline $\mathrm{C}$ & circulant matrix \\
\hline$d(n)$ & desired tequalizer output \\
\hline D & diagonal matrix of input signal power \\
\hline$\dot{\mathrm{D}}$ & estimate of $D$ \\
\hline$c(n)$ & scalar error at the equalizer output \\
\hline $\mathrm{e}(n)$ & error vector at the equalizer output \\
\hline $\mathbf{e}_{\mathcal{F}}(n)$ & Fourier-transformed error vector \\
\hline$E_{B}$ & rnergy per data bit \\
\hline $\mathcal{F}$ & DFT matrix \\
\hline$f(t)$ & $\begin{array}{l}\text { combined impulse response of the transmitter. channel and the } \\
\text { receive filter }\end{array}$ \\
\hline$h(t)$ & $\begin{array}{l}\text { combined impulse response of the transmit filter and the chan- } \\
\text { nel }\end{array}$ \\
\hline$h_{t r}(t)$ & transmit filter impulse response \\
\hline$h_{r \cdot r}(t)$ & receive filter impulse response \\
\hline$h_{c}(t)$ & channel impulse response \\
\hline$h_{R C}(t)$ & raised cosine impulse response \\
\hline$h_{R R C}(t)$ & root raised cosine impulse response \\
\hline$g_{k}$ & samples of the overall equalized channel response \\
\hline$I_{k}$ & transmitted symbols \\
\hline$\dot{I}_{k}$ & detector output \\
\hline
\end{tabular}




\begin{tabular}{|c|c|}
\hline$J(n)$ & méan square error \\
\hline$J_{B}(n)$ & block mean square error \\
\hline Irsen & t'xcess mean square error \\
\hline$J_{m ! n}$ & mininum mean squtare error \\
\hline$J_{\tau}(n)$ & transformed mean square error \\
\hline$J \dot{\tau}(n)$ & normalized transformed mean square error \\
\hline $\mathrm{K}$ & arror block constraint matrix \\
\hline$L$ & data block size \\
\hline - & adaptation misadjustment \\
\hline$\therefore$ & unumber of equnalizer taps \\
\hline$P_{i j}$ & complex noise PSD at the chamnel output \\
\hline $\mathrm{p}$ & cross-correlation of input bits with the desired response \\
\hline $\mathbf{P}$ & gradient constraint matrix \\
\hline $\mathrm{q}_{2}$ & rigenvector of the input data auto-correlation matrix \\
\hline$Q(\cdot)$ & complementary error function \\
\hline $\mathrm{R}$ & input data auto-correlation matrix \\
\hline $\mathrm{R}_{F}$ & Fourier-transformed input datat auto-correlation matrix \\
\hline $\mathbf{R}_{\tau}$ & transformed input data auto-correlation matrix \\
\hline $\mathbf{R}_{\bar{\tau}}^{\cdot \cdot}$ & normalized transformed input data auto-correlation matrix \\
\hline$T$ & simbol cluration \\
\hline$T_{F}$ & Fourier series period for series computation of $B E R$ \\
\hline $\mathcal{T}$ & unitary transformation matrix \\
\hline $\mathrm{v}(n)$ & $\begin{array}{l}\text { dlifference between equalizer taps and the optimum equalizer } \\
\text { solution }\end{array}$ \\
\hline $\mathbf{v}_{\tau}(n)$ & transformed vector $\mathbf{v}(n)$ \\
\hline$v_{\tau}^{\circ}(n)$ & normalized transformed vector $\mathrm{v}(n)$ \\
\hline
\end{tabular}




\begin{tabular}{|c|c|}
\hline$v_{i L T}(n)$ & LLT-transformed rector $\mathbf{v}(n)$ \\
\hline $\mathbf{w}(n)$ & requalizer tap weight rector \\
\hline$w_{F}=(n)$ & Fourier-transformed equalizer tap vector \\
\hline $\mathbf{w}_{T}\{n\}$ & transformed ecqualizer rap) rector \\
\hline$w_{i}^{*}(n)$ & normalized transformed equalizer tap vetor \\
\hline $\mathbf{w}_{1}$ & optimum equalizer tap vector \\
\hline $\mathbf{x}(n)$ & equalizer input vector \\
\hline$x=(n)$ & Fourier-transformed equalizer input vector \\
\hline $\mathbf{x}_{r}(n)$ & transformed equalizer input vector \\
\hline$x_{T}^{-1}(n)$ & normalized transformed equalizer input rector \\
\hline $\mathrm{X}_{-}=(k)$ & $\begin{array}{l}\text { liagonal matrix of the Fourier-transformed equalizer input sam- } \\
\text { ples }\end{array}$ \\
\hline$y(m)$ & sc'allar repualizer ontput \\
\hline $\mathbf{y}(n)$ & epualizer output vertor \\
\hline$y_{-}=(11)$ & Fourier-transformed equalizer output vector \\
\hline .3 & "xe'ss bandwidth factor of the raised cosine pulse \\
\hline$\hat{\theta}$ & smoothing factor for irerative signal power estimation \\
\hline$\delta(\cdot)$ & Dirac delta function \\
\hline$\Delta$ & BER series error \\
\hline$\lambda_{1}$ & eigenvalue of the input autu-correlation matrix \\
\hline .1 & $\begin{array}{l}\text { diagonal eigenvalue matrix for the input auto-correlation ma- } \\
\text { trix }\end{array}$ \\
\hline$\mu$ & adaptation step size \\
\hline$\mu_{B}$ & block adaptation step size \\
\hline$\rho$ & auto-correlation of input data sample \\
\hline$\sigma_{r}^{2}$ & noise variance at the equalizer input \\
\hline$\sigma_{\Xi}^{2}$ & noise variance at the equalizer output \\
\hline
\end{tabular}




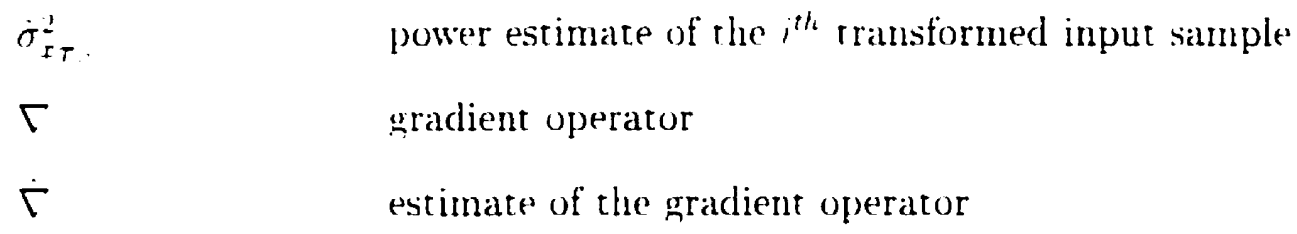

xvi 


\section{Chapter 1}

\section{Introduction}

\subsection{Motivation}

For time dirision multiple access (TDMA), the fundamental challenge in high data rate wireless transmission is overcoming intersymbol interference (ISI) caused by multipath propagation. Along with cell sectorization. beamforming and various diversity rechniques. time domain equalization has been the most common method of mitigating ISI. As the signaling rate increases. the number of dispersion symbols grows linearly with it. As a result. the number of equalizer cocfficients. $\lambda$. needed to eliminate ISI increases accordingly. Since the complexity per block of data of two common types of equalizers - linear and decision feedback. is on the order of $x^{-2}$. computational complexity presents a potential barrier. eren with todays state of digital signal processing technology:

Channels of interest in this thesis include those for high data rate applications. where the intersymbol interference length is large. Such cases require equalizers with a large number of taps. Using the common time domain techniques to compensate for distortion introduced br such channels may be rery computationally intense. Frequency domain equalization is a technique that offers low complexity growth with an 
increase of equalizer length in comparison to the time domain approach. Brefficienty. implementing block data processing with the use of the fast Fourier transform (FFT).

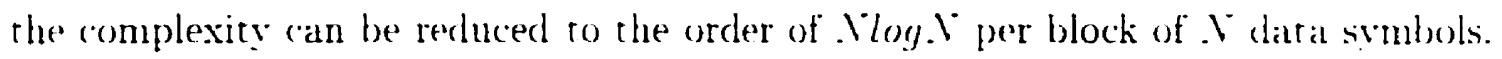
For a large number of equalizer taps this ramslates ro substantial computational savings.

This thesis presents an analysis of the frequency domain equalization as applied to a number of multipath channels. Performance measures such as the bit error rate. ronvergence' properties and computational complexity are examined and compared to the time domain equalizarion approach.

\subsection{Literature Review}

Adaptive equalizers were pioneered by Lucky in 196:5 ! 1!? ? who is credited for the development of the zero-forcing algorithm. This algorithm. based on the peak distortion criterion. led to the development of high speed modems. Concurrently to Luckys work. Widrow [3][4] developed the least mean square (L.MS) algorithm. which has been a popular choice in many applications.

The first frequency domain implementation of the L.IS algorithm was presented in 1973 by Waltzman and Schwartz [5]. who introduced an adaptation algorithm based on an isolated training sequence. The algorithm presented there included a gradient constraint implemented using Rosen's projection method. In 1978. Dentino et al. [6] proposed a frequency domain L.IS algorithm which updated the equalizer taps once per block of data. However. since proper data sectioning was not implemented. the algorithm performed circular convolution thus yielding a set of tap values different from the Weiner solution. It did offer significant decrease in computational complexity as no gradient constraint was included. The above shortcomings were finally overcome 
in 1980 by Ferrara [ 7 ! who recognized the effect of circular convolution and correctly applied overlap-save sectioning to correct it. Ferrara also implemented a aradient constraint. and the resulting frequency domain block L.MS algorithm converged to the proper set of tap ralues. The decelopment of the non-block frequency domain L.MS algorithm (referred to as sliding-DFT) is credited to Narayan [S!.

Since the initial development. a generalization of the frequency domain algorithm to a number of different transforms has been developed and their advantages discussed ! $9 ! 10 ![11]$. The convergence properties have been studied extensively and its dependence on the disparity of the input correlation matrix has been recognized 12 ! An improvement in the algorithm s learning curve has been obtained by normalizing the step size by the power distribution in the input spectrum. An excellent tutorial paper summarizing frequency domain adaptivo tiltering has been presented by Shrnk [1.3!.

Recently: Clark [14] hats applied the FBL.MS algorithm to a high data rate wireless environment. Promising performance over fatling channels has been obtained using the frequency domain approach with diversity combining. Clark's algorithm however used a cyclic prefix method of achieving linear convolution. which decreased the transmission efficiency. Clark [1t] states that for high data rate cases. resulting in large intersymbol interference and needing long equalizers. only the frequency domain method is practical. This thesis examines this claim for 1-4 Mbps transmission over a such channeis.

\subsection{Thesis Contributions}

We present a performance analysis of the normalized fast block least mean square (NFBL.MS) algorithm for high data rate channels. Unlike Clarks implementation. 
which uses a crelic prefix. the algnorithm uses overlap-sate data sectioning in order to ensure proper adaptation results.

The thesis offers a detailed derivation of the XFBL.IS algorithm hased on the roncepts of block processing and transform domain equalization. It is shown that. if properly implemented. this algorithm offers the advantages of reduced computational load and inproved convergence properties relative to the time domain equalization rechniques.

An NFBL.MS equalizer is applied to channels with short inpulse response. For such cases. We show that a fractionally-spaced expualizer is capable of performing the function of a receive tilter. For "ases where the length of the recoive tilter is comparable to that of the equalizer. the former is remoted and a fractionally-spaced equalizer is used. Due to the equalizer's low romplexity growth with the increase of the number of taps. such arrangement offers comparable computational complexity while enabling for a single structure receiver. Results are presented to show that such a receiver offers essentially equal bit error rate performance to a conventional cascade of a receive filter and a $T$-spaced equalizer.

We present a study of bit error rate (BER) performance for two high data rate channels and show that satisfactory results can be obtained up to 2.0 Mbps BPSK and 4.0 Mbps QPSK within a signal-to-noise ratio (S.VR) of $20 \mathrm{~dB}$. We confirm the findings by including an analysis of the equalized signal-to-noise-and-interference ratios (SNIR) which predict the BER results.

We show that for both types of channels considered. by normalizing the step size in the adaptation process according to the distribution of power across the input spectral bins. the rate of convergence of the frequency domain algorithm can be greatly increased in comparison to the L.IS algorithm. 
For high data rate channels. it is demonstrated that when coupled with a receive filter. the FBL.MS algorithm offers a considerable sarings in computational complexity orer the time domain L.IS algorithm by virtue of an efficient implementation of block processing.

\subsection{Thesis Outline}

The thesis is organized in the following manner. Chapter 2 discusses the theorerical background by tirst presenting frequency domain adaptive tiltering as a specific case of transform domain adaptive filtering. The equiralence of the frequency domain algorithm to a commonly known block adaptive algorithm is also demonstrated. Chapter 3 presents an analysis of performance measures for channels with relatively short impulse responses. including bit error rate. convergence properties and computational complexity analysis. Chapter $\downarrow$ examines the same performance criteria as applied to channels containing a large number of ISI terms that occur in high bit rate applications. Finally. Chapter $j$ contains a summary of the findings of this work along with presenting a number of suggestions for further work in the area. 


\section{Chapter 2}

\section{Theoretical Background}

\subsection{Introduction}

Adaptive equalization is one of many applications of the signal processing tool known as adaptive filtering. ()ther important uses include interference and echo cancellation. linear prediction and spectral estimation. While this thesis deals strictly with adaptive channel equalization. the following theoretical treatment. based mainly on [13].115].[16] and [17] is applicable to the general problem of adaptive filtering.

The primary purpose of this chapter is to derive the fast block least mean square (FBL.MS) equalization algorithm, which operates in the frequency domain. In doing so. we first introduce the most common steepest descent search method. the least mean square (L.MS) algorithm. The treatment covers an analysis of its computational complexity as well as detailed discussion of the convergence properties. The latter includes eigenanalysis. which gives a good insight into the effects of the eigenvalue spread of the input data correlation matrix on the adaptation learning curve. Is will be shown. the L.MS algorithm suffers from slow convergence in the case of highly disparate eigenvalues of the correlation matrix.

The concept of transform domain adaptive filtering (TDAF) is introduced as a 
solution to the poor convergence properties in case of high eigenvalue spread. W' show how by applying an orthogonal transformation to the correlation matrix the latter is diagonalized and subsequently normalized to reduce the eigenvalue disparity:

We present the concept of block adaptive filtering as an intermediate step in tho deritation of the FBL.MS algorithm. Lnlike the L.IS and TDAF algorithms, which use an instantenous value of the gradient in the adaptation process. the block L.MS (BL.MS) algorithm uses an average gradient estimate vver a block of data. which allows

for less noisy convergence. Block processing of data in the BLMS algorithm lends itself to a very efficient implementation via Fourier transform techniques. He show how the operations of correlation and convolution can be efficienty performed ria the fast Fourier transform (FFT). A distinction between linear and circular convolution is made and it is demonstrated how the undesirable wrap around effects inherent to convolution via the FFT (an be aroided provided proper data sectioning is performed. We describe two such methods: the overlap-sate and the overlap-add inchniques.

Finally we present the FBL.MS algorithm which utilizes the overlap-save method to efficiently carry out the operations of convolution and correlation. Apart from the savings in computational complexity, the FBL.MS inherits the fast convergence properties of transform domain equalization. provided that step size in each adaptation is normalized according to the input power distribution. The algorithm which uses the variable step size is referred to as the normalized FBL.IS (NFBL.MS).

\subsection{Wiener Filters}

A general filtering problem involves linear estimation of a desired signal based on a filter input sequence. The goal is to select the filter parameters such that the error of this estimation is minimized. The linear filter most often used for channel equalization 


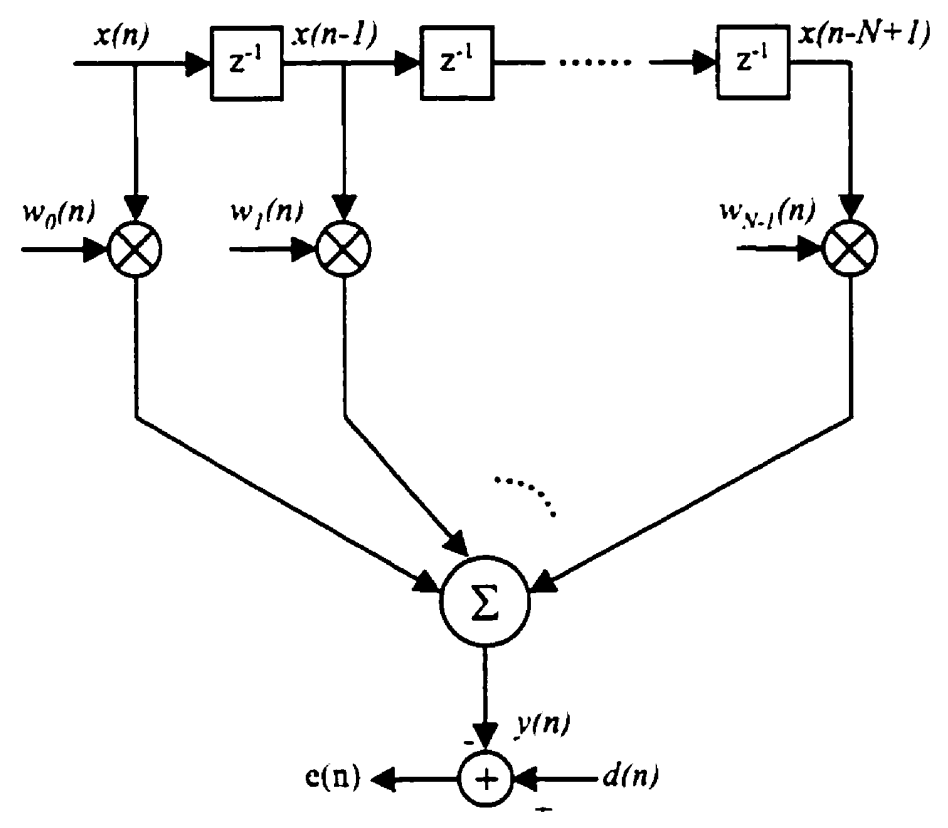

Figure 2.1: A Transiersal Filter

is a transversal filter depicted in Figure 2.1. The equalizer input and the tap weights are defined. respectively: as column vectors 1,5$][16]$

$$
\mathbf{x}(n)=\left[\begin{array}{llll}
x(n) & x(n-1) \quad \ldots & x(n-. r+1)
\end{array}\right]^{T}
$$

and

$$
\mathbf{w}(n)=\left[\begin{array}{llll}
u_{0}^{*}(n) & u_{i}^{*}(n) & \ldots & u_{i-1}^{*}(n)
\end{array}\right]^{T}
$$

where $n$ is the time index and the superscripts " and $T$ denote complex conjugation and a transpose. The output of the equalizer. denoted as $y(n)$. is the sum of the input samples weighted by the tap coefficients, that is

$$
\begin{aligned}
y(n) & =\sum_{i=0}^{r-1}\left(w_{1}(n) x(n-i)\right. \\
& =\mathbf{w}^{H}(n) \mathbf{x}(n) .
\end{aligned}
$$


where the superscript $H$ denotes a Hermitian transpose. The tap ralues are to be (hosen such that the output $y(n)$ closely approximates the desired signal which. in the case of channel equalization. is the transmitted symbol $d(n)$. The error of this astimation is defined as

$$
\cdot(n)=d(n)-y(n) .
$$

The process of selecting the optimum tap values is usually done by optimizing some kind of performance function. The criterion most commonly used is the mean square frror (.MSE). defined as

$$
J(n)=E !(n)(n) ! !^{2} .
$$

where $E[\cdot j$ and i| $\cdot \|$ denote statistical expectation and the norm operations respectively: If we define the cross-correlation vector $\mathbf{p}$ and the auto-correlation matrix $\mathbf{R}$ respectively as

$$
\mathrm{p}=E\left[\mathbf{x}(n) d^{*}(n) !\right.
$$

and

$$
\mathbf{R}=E\left[\mathbf{x}(n) \mathbf{x}^{H}(n)\right]
$$

the XSE can then be expressed as

$$
J(n)=E\left[d^{2}(n)\right]-2 \mathbf{w}^{H}(n) \mathbf{p}+\mathbf{w}^{H}(n) \mathbf{R} \mathbf{w}(n) .
$$

It can be shown [15][16] that the USE as a function of $\mathbf{w}(n)$ forms a surface in $\lambda+1$ dimensional Euclidean space. referred to as the performance surface. Furthermore. since the auto-correlation matrix $\mathbf{R}$ is positive definite. this surface is convex [15][16]. Its global minimum corresponds to the optimum tap values. that is those minimizing 
the IISE. This point. defined as $\mathbf{w}(n)=\mathrm{w}_{0}$. can be obtained by setting the partial derivatives of $J$ with respect to the tap weights to zero. that is

$$
\Gamma \cdot J(n)=0 .
$$

where $\Gamma$ is the gradient defined as

$$
\nabla=\left[\begin{array}{llll}
\frac{d}{\left.\partial u^{\prime}\right)} & \frac{\partial}{\partial u^{\prime} !} & \cdots & \frac{\partial}{1 \pi u^{\prime} x-1}
\end{array}\right]^{T} .
$$

The solution to (2.9) is the well known Wiener-Hopf equation [15][18!

$$
R \mathbf{w}_{o}=\mathrm{p} .
$$

where $\mathbf{p}$ and $\mathrm{R}$ are detined $\mathrm{by}(2.6)$ and (2.7).

\subsection{The LMS Algorithm}

Solving the Wiener-Hopf equation requires inverting the auto-correlation matrix $\mathbf{R}$. An alternative way of minimizing the MSE avoids this operation by solving for the optimum tap values iteratively: The most common of such techniques is the method of steepest descent [18]. where the solution is obtained by starting at an arbitrary point on the performance surface. $w(0)$, and progressively moving towards the global minimum. Since the function is convex. moving in the direction of the steepest slope guarantees reaching the optimum solution. At each iteration. a gradient of the function is computed as

$$
\begin{aligned}
\Gamma \cdot J(n) & =\frac{\partial J(n)}{\partial \mathbf{w}(n)} \\
& =2 \mathbf{R} \mathbf{w}(n)-2 \mathbf{p} \\
& =-2 E\left[\mathbf{x}(n) \epsilon^{*}(n)\right]
\end{aligned}
$$




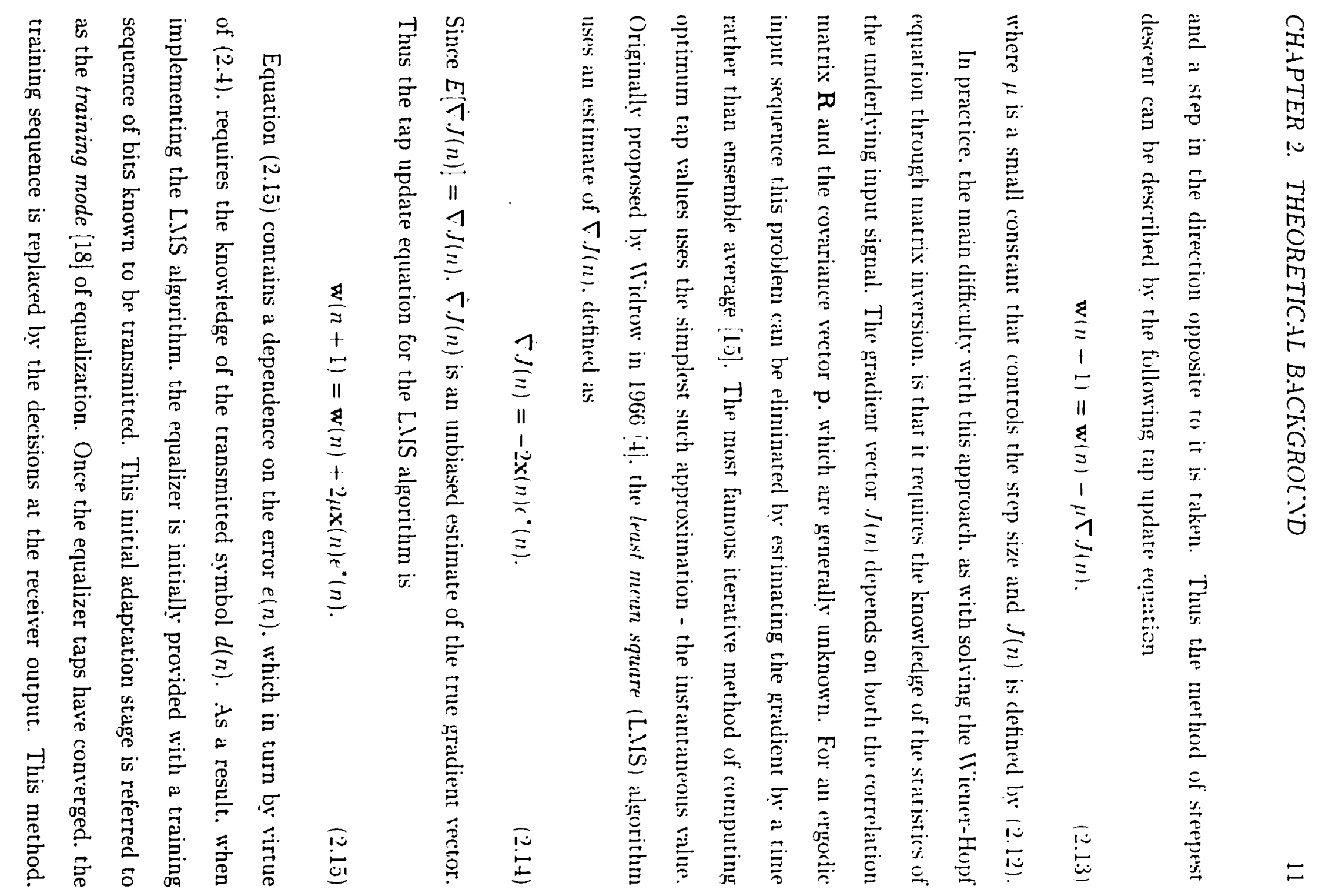


referred to as automatic or decision-directed [18! equalization. also allow's the equalizer to track slow changes in the channel response.

\subsubsection{Computational Complexity of the LMS Algorithm}

In describing the computational complexity of the' algorithm. we examine the number of real multiplications necessary to process a data block of length .1 . Although other facrors. such as the number of additions and storage requirements. also contribute to the complexity the analysis presented here provides a reasonably accurate estimate.

For a real input signal. the adaptive equalizer of length .1 implemented using the L.MS algorithm requires .1 real multiplications to compute the output and another $\therefore$ real multiplications to compute the gradient per sample. Thus. processing a block of .1 real input samples requires a total of $2.1^{\prime 2}$ real multiplications.

In the case where the input signal is complex. the equalizer taps are also complex valued. Since each complex multiplication is repivalent to 4 real multiplications. the complexity increases by a factor of 4 . resulting in $8.1 \%$ multiplications per block of data.

\subsubsection{Convergence Properties of the LMS Algorithm}

It is well known [12] that the eigentalue disparity of the input signal correlation matrix $\mathbf{R}$ determines the rate of convergence of the gradient descent method. In order to examine this property in more detail. it is helpful to express the equalizer tap coefficients $\mathbf{w}(n)$. in terms of the eigenvalues of $\mathbf{R} . \lambda_{i}$. and the corresponding eigenvectors $\mathrm{q}_{i}$. This relationship can be expressed as [15]

$$
w(n)=w(0)+\sum_{i=0}^{N-1} \alpha_{i}\left(1-2 \mu \lambda_{i}\right)^{n} \mathbf{q}_{i} .
$$


where $a$, is a constant. A similar relationship can be obtained for the IISE. namely

$$
J(n)=J_{\min }+\sum_{i=11}^{x-1} a_{i}^{2} \lambda_{t}\left(1-2 \mu \lambda \lambda_{1}\right)^{2 n} .
$$

The above expressions indicate that the convergence of the equalizer taps for the gradient descent method. and thus for the L.IS algorithm. is governed by a sum of $\therefore$ exponential terms each being a function of a separate eigenvalue $\lambda_{2}$. Every term independently controls onte mode of convergence in the direction of its eigenvector $\mathrm{q}_{i}$. Thus. the convergence is rapid when $\mid 1-2 \mu h \lambda_{2} !$ is small. In addition. from $(2.16)$ and (2.17) we note that in order to ensure the srability of the L.IS algorithm. the step size $\mu$ must satisfy $0<\mu<1 / \lambda_{\max }$. These two conditions are impossible to satisfy if the eigenvalue spread $\lambda_{\operatorname{trax}} / \lambda_{\min }$ is large $[18$ !

The dependence of the convergence rate on the spread of the eigenvalue $\lambda_{\max } / \lambda_{\min }$ translates to a dependency on the power spectral density of the filter input. Yore specifically: the eigenvalues of the correlation matrix are bounded by the minimum and maximum values of the power spectral density of the input process [15]. When the filter input is white. that is the spectrum is flat across the entire range of frequencies. the L.IS algorithm converges rapidly. However. in the case where the spectrum contains nulls. some of the modes converge slowly: thus greatly reducing the orerall rate of convergence.

A geometrical interpretation of the above properties can be obtained by examining the shape of the performance surface as a function of the eigenvalues and eigenvectors of $\mathbf{R}$. L sing (2.11). the MSE can be rewritten as

$$
J(n)=J_{\min }+\left(\mathbf{w}(n)-\mathbf{w}_{o}\right)^{H} \mathbf{R}\left(\mathbf{w}(n)-\mathbf{w}_{o}\right) .
$$

where the minimum IISE. $J_{\min }$. is given by

$$
J_{\min }=E\left[d^{2}(n)\right]-\mathbf{w}_{n}^{H} \mathbf{R w}_{o} .
$$


The positive definiteness of the matrix $\mathbf{R}$ implies that the second term in (2.18) is always non-negative, and equal to zero only at the point $w(n)=\mathrm{w}_{0}$. that is. corresponding to $J(n)=I_{\min }$. Thus. $J(n)$ is a convex function of tap weights. By defining a rector

$$
\mathbf{v}(n)=\mathbf{w}(n)-\mathbf{w}_{\imath} .
$$

(2.18) (an be rewritten ats

$$
J(n)=J_{\min }-\mathrm{v}(n)^{H} \mathrm{Rv}(n) .
$$

which is equivalent to shifting the origin of the Euclidean space to the point $\mathbf{w}_{0}$. Furthermore. $\mathbf{R}$ can be decomposed using the unitary similarity transformation [15]

$$
\mathrm{R}=\mathrm{Q} \cdot \mathrm{IQ}^{H} .
$$

where the matrix $\perp$ is diagonal with the eigenualues of $R . \lambda_{2}$. as its entries, while the columns of the matrix $Q$ consist of the corresponding orthonormal eigenvectors $q_{1}$. If we define

$$
\mathbf{v}_{K L T}(n)=\mathbf{Q}^{H} \mathbf{v}(n) .
$$

in which the subscript KLT refers to what is known as the Karhunen-Loéte Transform. (2.21) can be expressed as

$$
\begin{aligned}
J(n) & =J_{\min }+\mathrm{v}_{K L T}(n)^{H} \cdot \Delta \mathrm{v}_{K L T}(n) \\
& =J_{\min }+\sum_{i=0}^{V i-1} \lambda_{t} r_{k L T \cdot t}^{2}(n) .
\end{aligned}
$$

Equation (2.23) is equivalent to rotating the axes such that they point in the direction of the eigenvectors of $R$. Thus the performance surface is in the shape of a 
hyperparabola in $\lambda+1$ dimensional space whose axes are the $X^{*}$ ralp-weight rariables and the MSE. The contours of this surface. corresponding to a specific value of $J(n)$. consist of hyperellipses whose principal axes lie in the directions of the eigenvectors of R. It can be shown 1 lji that the size of each principal axis is inversely proportional ro the square root of the corresponding eigenvalue. Thus the larger the eigenvalue spread. the greater the eccentricity of the hyperparabolas.

\section{Misadjustment of the LMS algorithm}

Another property that should be taken into consideration when examining the learning curve of an adaptive algorithm is the fuctuation of the tap values around the optimum solution at steady state. The steady state error of the algorithm is referred

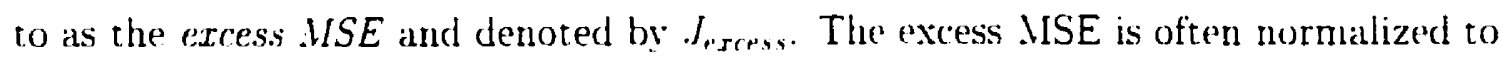
$f_{\text {rmm }}$. the result given the term misadjustment

$$
\mathcal{M}=\frac{J_{\text {trees: }}}{. J_{n+n}}
$$

For the L.MS algorithm. one can show 15 that for misadjustment values of $10 \%$ or less

$$
U_{L, U S}=\mu \operatorname{tr}[\mathbf{R} !
$$

where $\operatorname{tr}[\cdot]$ refers to the trace operation.

\subsection{Transform Domain Equalization}

As pointed out previously: the convergence of the L.MS algorithm is controlled by $\checkmark$ distinct modes. each characterized by an eigenvalue of the correlation matrix $\mathbf{R}$. Since the eigenvalue spread is directly linked to the power spectral density of the input process. the convergence behaviour is also dependent on the spectral characteristics 
of the input process. The LXIS algorithm will converge rapidly in the frequency bands where the adaptive filter is well excited. and slowly in the poorly excited bands. An intuitive solution to this problem would be to employ a set of bandpass filters to separate the input into subbands and subsequently use a normalization process to equalize the energy content in each band. The signal normalized in such manner can then be used for the adaptation process of the equalizer taps.

Transform domain equalization. which was first proposed by Narayan and Peterson in 1981 [8]. implements the above procedure. It uses an orthogonal transform to partition the input data into separate frequency bins. following which the step size in the recursion is normalized according to their power content.

A diagram of a transform domain adaptive filter is shown in Figure 2.2. The input vector $\mathbf{x}(n)$ is transformed into a new set of samples by multiplying it by an unitary matrix $\mathcal{T}$. Defining the transformed vector as

$$
\begin{aligned}
\mathbf{x}_{\tau}(n) & =\mathcal{T} \mathbf{x}(n) \\
& =\left[\begin{array}{lllll}
x_{T .0}(n) & x_{T .1}(n) & \ldots & x_{T . .1-1}(n)
\end{array}\right]^{T}
\end{aligned}
$$

and the similarly transformed equalizer taps as

$$
\begin{aligned}
\mathbf{w}_{\mathcal{T}(n)} & =\mathcal{T} \mathbf{w}(n) \\
& =\left[\begin{array}{lllll}
u_{\mathcal{T} .0}^{*}(n) & u_{\mathcal{T}, l}^{*}(n) & \ldots & u_{\mathcal{T} . .,-1}^{*}(n)
\end{array}\right]^{T} .
\end{aligned}
$$

the output of the filter at time $n$ is given by

$$
\begin{aligned}
y(n) & =\sum_{i=0}^{i-1} u_{\tau, l}(n) \cdot x_{\tau_{, n-t}(n)} \\
& =\mathbf{w}_{\tau}^{H}(n) \mathbf{x}_{\mathcal{T}}(n) .
\end{aligned}
$$

Once the input data has been partitioned by the transform $\mathcal{T}$. the step size for each spectral component is set inversely proportional to the power content at that 


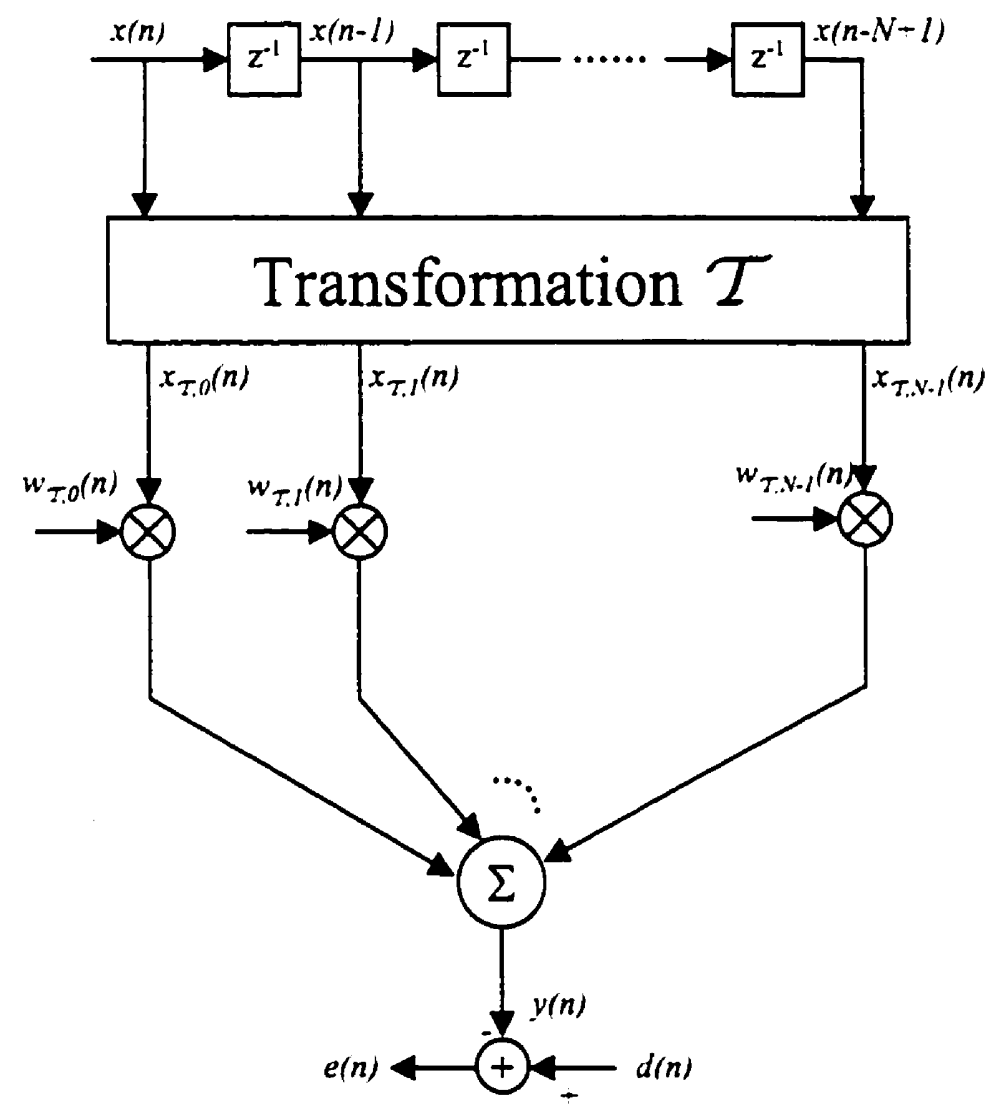

Figure 2.2: Transform Domain .tdaptive Filter 
frequency: This is equiralent to replacing the step size $\mu$ with $\mu \mathrm{D}^{-1}$ where $\mathrm{D}$ is the diagonal matrix with power estimates as it entries. that is

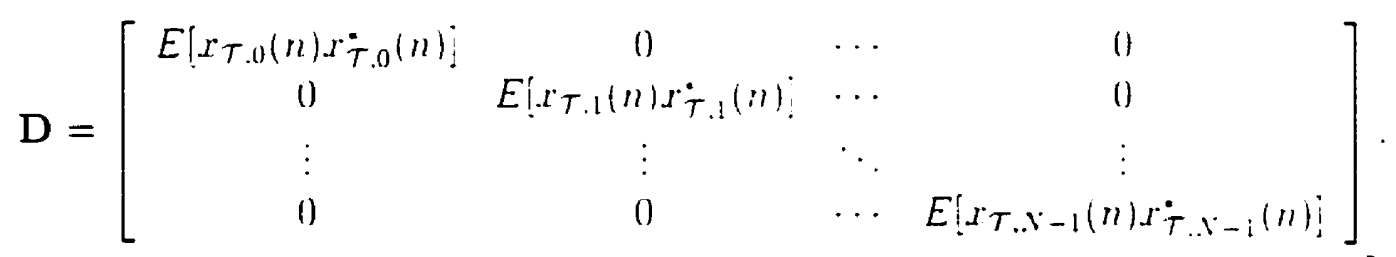

In practice. the matrix $\mathrm{D}$ is replaced by $\mathrm{D}$ with estimates of $E\left[x_{T_{. t}}(n), x_{\mathcal{T} . t}^{*}(n)\right]$ on its main diagonal. Each entry in $\hat{D}$ is computed by a recursion formula [15)

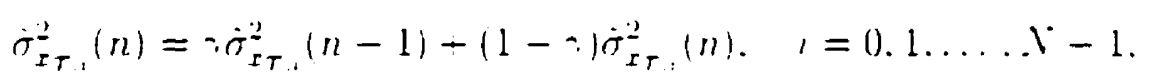

where - is often referred to as the smoothing factor. The resulting recursion formula for the TDL.MS algorithm is thus given by

$$
\mathbf{w}_{\mathcal{T}}(n+1)=\mathbf{w}_{\mathcal{T}}(n)+2 \mu \hat{\mathbf{D}}^{-1} \mathbf{x}_{\mathcal{T}}(n) \epsilon^{*}(n) .
$$

To show how this algorithm normalizes the power in each input bin to unity. (2.32) (an be decomposed into separate equations for each equalizer tap.

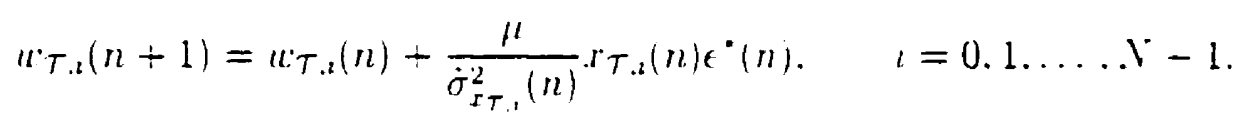

We can see from the above expression that the step size for each tap is inversely proportional to the corresponding power estimate. As a result the weakly excited subbands will have a larger step size. thus speeding up their convergence.

It can be shown that the transform domain L.IS algorithm is equivalent to the conventional L.IS algorithm with the normalized tap input vector. defined as $\mathbf{x}_{\mathcal{T}}^{\circ}(n)$. where

$$
\mathbf{x}_{\tau}^{\imath}(n)=\hat{\mathbf{D}}^{-1 / 2} \mathbf{x}_{\tau}(n)
$$


Furthermore. if we define

$$
\mathbf{w}_{\tau}^{\circ}(n)=\hat{\mathbf{D}}^{\prime} \mathbf{w}_{\tau}(n)
$$

and multiply (2.32) by $\hat{D}^{1,2}$. We obtain

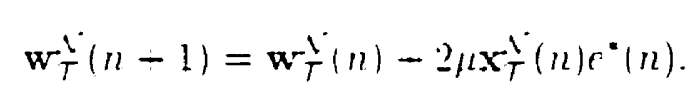

Noring that

$$
\begin{aligned}
c(n) & =d(n)-y(n) \\
& =d(n)-\mathbf{w}_{\tau}^{\prime \prime}(n) \mathbf{x}_{\tau}(n) \\
& =d(n)-\mathbf{w}_{\tau}^{\cdot}{ }^{H}(n) \mathbf{x}_{\tau}^{\prime}(n)
\end{aligned}
$$

shows that (2.32) is equivalent to the L.MS algorithm with the normalized input vector $\boldsymbol{x}_{\tau}^{\circ}(n)$.

\subsubsection{Computational Complexity of the TDLMS Algorithm}

While the TDL.IS algorithm was developed mainly to improve the rate of convergence. it exhibits a tradeoff in the form of increased computational complexity. The computation of the transform requires on the order of $\lambda^{13}$ multiplications. with the exact number depending on the type of input data and the transformation used.

\subsubsection{Convergence Properties of the TDLMS Algorithm}

As discussed in the Section 2.3.2. the eigenvalue spread affects the shape of the performance surface. which in turn dictates the speed of convergence. To examine the effect of applying the transformation to the input data on the shape of the surface. we recall that the performance surface without any transformation can be expressed 
ass

$$
J(\mathrm{v})=J_{\min }-\mathrm{v}(n)^{H} \mathrm{Rv}(n)
$$

where $\mathbf{v}(n)$ is defined $b r(2.20)$ and $\mathrm{R}$ is the input data correlation matrix. To compare this function to that for a transformed input sequence we define a transformed vector $\mathrm{v}_{\tau}(n)$ as

$$
\mathbf{v}_{T}(n)=\mathcal{T} \mathbf{v}(n)
$$

and the auto-correlation matrix of the transformed input sequence as

$$
\mathbf{R}_{\mathcal{T}}=\mathcal{T} \mathbf{R} \mathcal{T}^{H}
$$

The performance surface for at transformed input sequence $\mathrm{x}_{\mathcal{T}}(n)$ is then

$$
J_{\mathcal{T}}\left(\mathbf{v}_{T}\right)=J_{\min }-\mathbf{v}_{\tau}(n){ }^{H} \mathbf{R}_{T} \mathbf{v}_{T}(n)
$$

Substituting $(2.39)$ and $(2.40)$ vields

$$
\begin{aligned}
J_{\mathcal{T}}\left(\mathrm{v}_{\mathcal{T}}\right) & =J_{\min }-\mathbf{v}^{H}(n) \mathcal{T}^{H} \mathcal{T R} \mathcal{T}^{H} \mathcal{T} \mathbf{v}(n) \\
& =J_{\min }-\mathbf{v}^{\prime \prime}(n) \mathrm{R} \mathbf{v}(n) \\
& =J(\mathbf{v}) .
\end{aligned}
$$

where we have used the fact that $\mathcal{T}^{H} \mathcal{T}=\mathrm{I}$ for any unitary transformation matrix [15]. This shows that the performance surface in both cases is identical. regardiess of the transform used. The only effect of the transform is rotating the coordinate axis according to (2.39). which does not in itself affect the convergence rate.

If. however. in addition to the transformation. power normalization is performed. (2.41) becomes

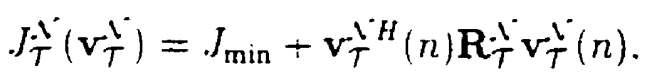


where

$$
\underline{v}_{\tau}^{\prime}(n)=\mathrm{D}^{1} \mathrm{v}_{\tau}(n)
$$

and

$$
\mathrm{R}_{\mathcal{T}}^{\cdot \cdot \dot{*}}=\mathrm{D}^{-1 \cdot 2} \mathrm{R}_{\mathcal{T}} \mathrm{D}^{-12}
$$

Substituting the abore expressions into (2.41). we obtain

$$
\begin{aligned}
& J_{T}^{\prime \prime}\left(\mathbf{v}_{T}^{-1}\right)=I_{\operatorname{man}}-\mathbf{v}_{T}^{H}(n) \mathrm{D}^{1 \cdot 2} \mathrm{D}^{-1 \cdot 2} \mathbf{R}_{\mathcal{T}} \mathrm{D}^{-1 / 2} \mathrm{D}^{1 \cdot 2} \mathbf{v}_{\tau}(n) \\
& =J_{\text {m1: }}+\mathbf{v}_{\tau}^{H}(n) \mathbf{R}_{\tau} \mathbf{v}_{\tau}(n) \\
& =J_{\mathcal{T}}\left(\mathbf{v}_{\mathcal{T}}\right) \\
& =J_{\mathcal{T}}\left(\mathrm{D}^{-1,2} \mathrm{v}_{T}^{\prime} \dot{*}\right) \text {. }
\end{aligned}
$$

The above relationship demonstrates that the performance surface for the normalized case is equiralent to that defined by (2.43). but with the coordinate axes scaled according to (2.44). This has the effect of reducing the ecrentricity of the hyperparabola. thus increasing the rate of convergence.

The most important task in implementing the TDL.IS algorithms is the selection of the transform $\mathcal{T}$. such that the elements of the transformed input vector have mutually exclusive spectral bands. Ideally: one would like to obtain a vector $\mathbf{x}_{\mathcal{T}}(n)$ with uncorrelated components [19]. which in turn results in a diagonal correlation matrix. Proper normalization would then yield an identity matrix with eigenvalue spread of unity: A transform with such properties is the Karhunen-Loeve Transform $(\mathrm{KLT})$. defined as $[15]$

$$
\mathcal{T}_{\text {KLT }}=\mathrm{Q}^{H} .
$$


where $\mathrm{Q}^{H}$ is a matrix with columns equal to eigenvectors of the correlation matrix R. as in the unitary similarity transformation. Recalling that $\mathbf{R}$ can be decomposed as

$$
\mathbf{R}=\mathrm{Q} \cdot 1 \mathrm{Q}^{H}
$$

we have

$$
\begin{aligned}
\mathbf{R}_{\tau_{\Lambda L T}} & =E ! \mathbf{x}_{T_{\Lambda L H}(n) \mathbf{x}_{\tau_{k L T}}^{H}(n) !} \\
& \left.=E ! \mathbf{Q}^{H} \mathbf{x}(n) \mathbf{x}^{H}(n) \mathrm{Q}\right] \\
& =\mathrm{Q}^{H} \mathrm{RQ} \\
& =\mathrm{Q}^{H} \mathrm{Q} \cdot 1 \mathrm{Q}^{H} \mathrm{Q} \\
& =.1 .
\end{aligned}
$$

The above shows that the altuto-correlation matrix of the KLT-transformed sequence is diagonal. If $\hat{D}$ is a good estimate of $D$. we have that the nurmalized correlation matrix is

$$
\begin{aligned}
\mathrm{R}_{\tau_{K L T T}}^{\cdot \cdot} & =\hat{\mathbf{D}}^{-1 / 2} \mathrm{R}^{-1 / 2} \\
& \simeq I .
\end{aligned}
$$

While the KLT is an ideal choice for the transform $\mathcal{T}$. as it produces an uncorrelated input sequence. it requires the knowledge of $R$. which is not alwars arailable. Therefore in practice other transforms. such as the Discrete Fourier Transform (DFT) or the Discrete Cosine Transform (DCT). are often used.

\subsection{Block LMS Algorithm}

The algorithms presented thus far use an instantaneous value of the gradient as an approximation to its statistical expectation. Such estimations are noisy and cause 
random fluctuations of taps during the adaptarion process. One method of improving the gradient estimate is to use a time average orer $L$ samples

$$
\dot{\Gamma} J_{H}(n)=-\frac{2}{L} \sum_{i=1)}^{L-1} \mathbf{x}(n+1) \epsilon^{\cdot}(n+i)
$$

where the subscript $B$ denotes that the gradient is estimated based on a block of $L$ data samples. The above is referred to as the block L.IS (BL.IS) algorithm. Since the gradient is updated every $L$ samples. the raps are held constant for the duration of the block. The output of the BL.MS equalizer is given by

$$
y(n-\imath)=\mathbf{w}^{H}(n) \mathbf{x}(n+i) . \quad,=0 \ldots . L-1 .
$$

The resulting tap update equation is

$$
\begin{aligned}
\mathbf{w}(n+L) & =\mathbf{w}(n)-\mu_{B} \dot{\Gamma} J_{B}(n) \\
& =\mathbf{w}(n)-2 \mu_{B} \sum_{i=1}^{l--1} \mathbf{x}(n-i) e^{\cdot}(n+i) .
\end{aligned}
$$

where $\mu_{B}=\mu L$ is the block step size. Since the BL.MS algorithm operates at a lower sampling rate than that of the input sequence. it is convenient to define a new time index $k$ one increment of which corresponds to $L$ increments in the original index $n$. Equation 2.53 can then be rewritten as

$$
\mathbf{w}(k+1)=\mathbf{w}(k)+2 \mu_{B} \sum_{i=0}^{L-1} \mathbf{x}(k L+i) e^{\bullet}(k L+i) .
$$

where the indices $n$ and $k$ are related by

$$
n=k L+i . \quad i=0 \ldots . L-1 .
$$

\subsubsection{Convergence Properties of the BLMS Algorithm}

Since the BL.MS algorithm computes a more accurate estimate of the gradient. the descent direction at each step is more aligned with the true gradient. thus resulting 
in smoother convergence [13]. This improvement becomes greater with a larger block size. However. when choosing the block length $L$. it is important to realize that since the taps are updated every $L$ samples. a delate is introduced at the output of the filter. If $L$ is large. the tracking performance of the BL.MS algorithm mat degrade.

In this thesis. we are mainly interested in the case where $L=\lambda$. since. as it will be shown. it allows for an efficient implementation of the BL.IS algorithm. The rate of convergence does not change. since much like in the case of the L.IS algorithm. it is governed by the eigentalue spread of the input datal correlation matrix. which is the same for both algorithms :15i.

\subsubsection{Computational Complexity of the BLMS Algorithm}

The complexity of the BLMS algorithm is the same as that of the L.IS algorithm. That is. it requires. respectively $2.9^{\prime 2}$ and $8.1^{\prime \prime 2}$ real multiplications per real and complex data blocks of length.. . However. there exists a very efficient implementation of the algorithm using DFT's. which offers a significant computational sarings. and which is described in the following section.

\subsection{Fast Block Least Mean Square Equalizers}

While transform domain adaptive filtering improves the convergence properties by reducing the eigenvalue spread of the data correlation matrix. it does it at the expense of increasing the computational complexity. In applications requiring large number of equalizer taps such complexity can be prohibitive. In this section we show how a computationally efficient implementation of BL.MS algorithm can be achieved in the frequency domain [13][15].

Equation (2.52) is essentially a linear convolution of the data vector with the 
equalizer taps. while the block gradient in (2.51) is a linear correlation of the error signal with the inpur datat. It is known from digital signal processing theory 17 . that these can be efficiently pertormed ria the discrete Fourier transform (DFT). However. since the DFT is a signal representation in terms of frequency samples. it corresponds to a periodic time signal. Inherently: this leads to (yclic (or circular) convolution/correlation which differs from the linear counterpart obtained by filtering sequences in the time domain. To correct this, there exist two common technicques of data sectioning that allow us to achiese linear convolution via the DFT. The following sections. based mainly on [1T] and [20]. discuss these algorithms and how they are incorporated into the FBL.IS algorithm.

\subsubsection{Linear and Circular Convolution}

Linear convolution of two sequences $x_{1}(n)$ and $x_{2}(n)$. both of length.$\lambda$. is given by

$$
\begin{aligned}
r_{3}(n) & =r_{1}(n) * r_{2}(n) \\
& =\sum_{m=-x}^{x} x_{1}(m) r_{2}(n-m) .
\end{aligned}
$$

where $x_{3}(n)$ is the resulting sequence of length $2.1-1$. Thus. $x_{3}(n)$ is obtained by multiplying one sequence by a time-reversed and linearly shifted version of the other and summing the values of the products for all $\mathrm{m}$.

We now examine the effects of computing convolution via the discrete Fourier

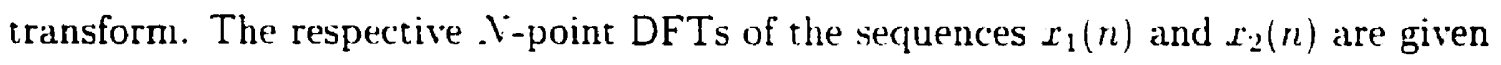
by

$$
x_{\mathcal{F} .1}(k)=\sum_{n=0}^{i-1} x_{1}(n) e^{-j 2 \pi k n \times x} \quad k=0.1 \ldots \ldots+1
$$

and

$$
x_{\mathcal{F} .2}(k)=\sum_{n=0}^{i \cdot-1} x_{2}(n) e^{-j 2 \pi k n / x} \quad k=0.1 \ldots \ldots+1 .
$$


Multiplving two DFTs rields a sequence $r_{.} F_{3}\left(k^{\circ}\right)$.

$$
r_{3.3}(k)=r_{E .1}(k) r_{E .2}(k) \quad k=0.1 \ldots \ldots t-1 .
$$

whose IDFT is given by

$$
\begin{aligned}
& r_{3}(n)=\frac{1}{N} \sum_{k=11}^{N-1} x_{F .3}(k) c^{j 2 \pi k n . .1}
\end{aligned}
$$

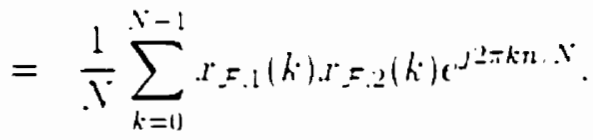

Substituting (2.57) and (2.58) into (2.60) we obtain

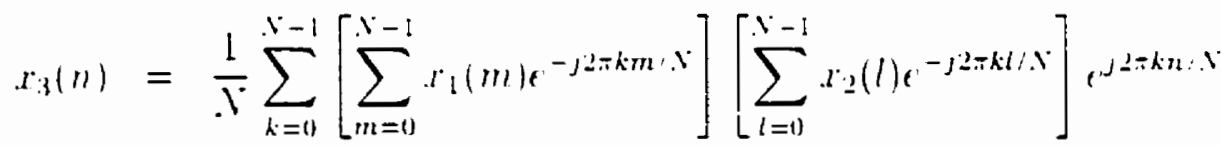

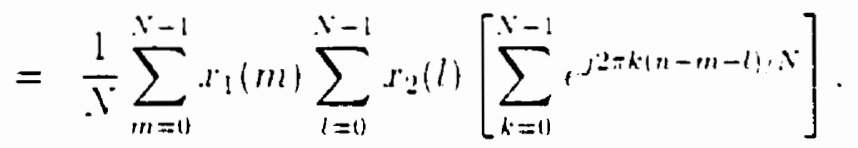

Let the third summation of $(2.61)$ be expressed as

$$
\sum_{k=1)}^{x-1} a^{k}=\left\{\begin{array}{ll}
x . & a=1 \\
\frac{1-a^{x}}{1-a} . & a \neq 1
\end{array} .\right.
$$

where $a$ is defined as

$$
a=s^{2+2}(n-m-1) x
$$

We can see that $a=1$ when $n-m-l$ is a multiple of .1 . Also. from (2.63) we have that $a^{x}=1$ for any value of $a \neq 0$. Thus $(2.62)$ can be expressed as

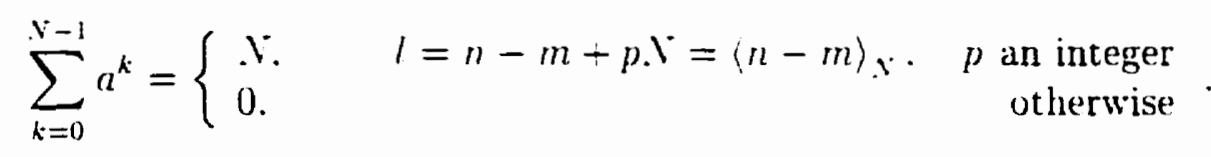

where $\langle\cdot\rangle_{x}$ denotes modulo- $\rangle^{\prime}$ arithmetic. Using (2.61) and (2.6t) we obtain an expression for $x_{3}(n)$

$$
\left.x_{3}(n)=\sum_{m=0}^{Y-1} x_{1}(m) x_{2}((n-m)\rangle_{N}\right)
$$


In contrast io linear convolution. here $r_{3}(n)$ is obtained by multiplying one sequence by a rime-reversed and corcularly shifted version of the other and summing values of the product for all $m$. Thus multiplying DFT's of $x_{1}\left(k^{\prime}\right)$ and $l_{2}\left(k^{\prime}\right)$ and raking the inverse DFT of the product corresponds to performing .9 -point etrcular convolution on $r_{1}(n)$ and $r_{2}(n)$. denoted as

$$
r_{3}(n)=r_{1}(n) \approx r_{2}(n) .
$$

In order to show how to pertorm linear convolution via the DFT. wo first note that frequency domain equivalent of the linear convolution. defined by (2.56). of sequences of lengths $L$ and $P$ is $[1]$

$$
X_{3}(\omega)=X_{1}(\omega) X_{2}(\omega)
$$

In order to represent the output sequence $x_{3}(n)$ in the frequency domain by the samples of its spectrum $I_{3}(\omega)$. the number of distinct samples must equal or exceed its length. that is $L+P-1$. Therefore. a DFT of length $N^{\circ} \geq L-P-1$ is needed to properly represent the sequence $x_{3}(n)$ in the frequency domain [1T]. Thus a linear convolution of sequences of lengths $L$ and $P$ can bo achieved by multiplying the respective DFT's provided that each DFT is of length $Y \geq L+P-1$. One way of ensuring this is to augment both sequences with an appropriate number of zeros. However. computing a convolution in such manner is impractical in situations when the length of one of the sequences is very large. since storing it would require a large buffer size. More importantly: since the filter output is not computed until all the samples have been collected. there is a large processing delay. For these reasons. most practical approaches involve breaking the input sequence into blocks and computing the output in segments which are then appropriately combined. This is referred to as block convolution. The most common methods of block filtering in the frequency 
clomain are the ouplap-sate and the overtap-ndd techniques presented below. In their discussion we assume that the long inpur data stream is broken down into blocks of length $L$. The filter is assumed to be of length $P$.

\section{Overlap-Add Method for Implementing Linear Convolution}

The orerlatp-add sectioning is depicted in Figure 2.3. In this method. the input blocks of length $L$ are padded with $P-1$ zeros. The filter response (of length $P$ ) is patded with $L-1$ zeros. to produce a secquence of roral length $V=L-P-1$. . V-point DFT's are computed and the IDFT of their product is taken. The individual output blocks are free from aliasing. since as described in the previous section. the DFT's are of length $. Y=L-P-1$. Since the input blocks contain $P-1$ zeros, the last $P-1$ points of each individual output must be overlapped and added to the first $P-1$ points of the next output block.

\section{Overlap-Save Method for Implementing Linear Convolution}

The overlap-save method is shown in Figure 2.t. Similar to overlap-add. this method also generates input data blocks of size $. Y=L-P-1$. Here. however. each data block consists of the last $P-1$ points from the previous block followed by $L$ new data points. Like in the case of overlap-add. the filter response of length $P$ is parded with $L-1$ zeros. Again. the IDFT of the product of the $Y$-point DFT's is computed. Since the first $P-1$ points of each individual output is a result of aliasing, it is simply discarded. while the last $L$ points of appended to the successive output blocks.

\subsubsection{Fast Block LMS Algorithm}

The most common implementation of the FBL.IS algorithm uses the overlap-save method to process the input bits. The filtering is equivalent to that discussed in the 


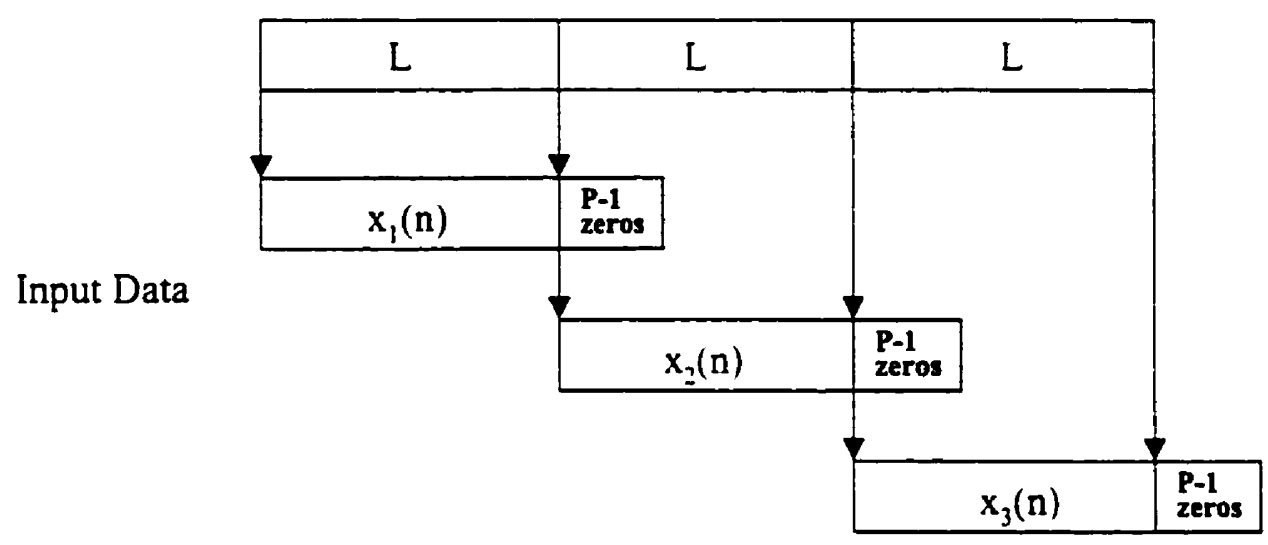

Filter

\begin{tabular}{|l|l|}
\hline $\mathrm{W}(\mathrm{n})$ & L $\mathrm{L}-\mathrm{i}$ \\
\hline
\end{tabular}

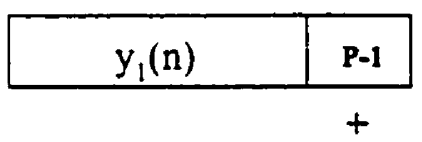

Output Data

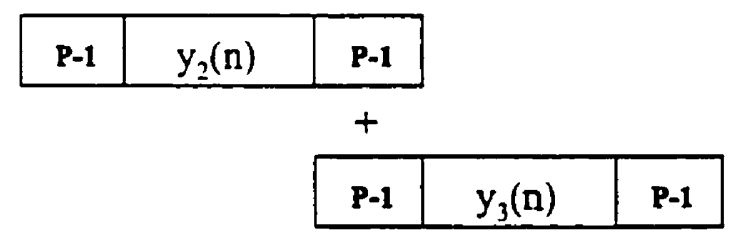

Figure 2.3: Overlap-Add Method of Linear Convolution 


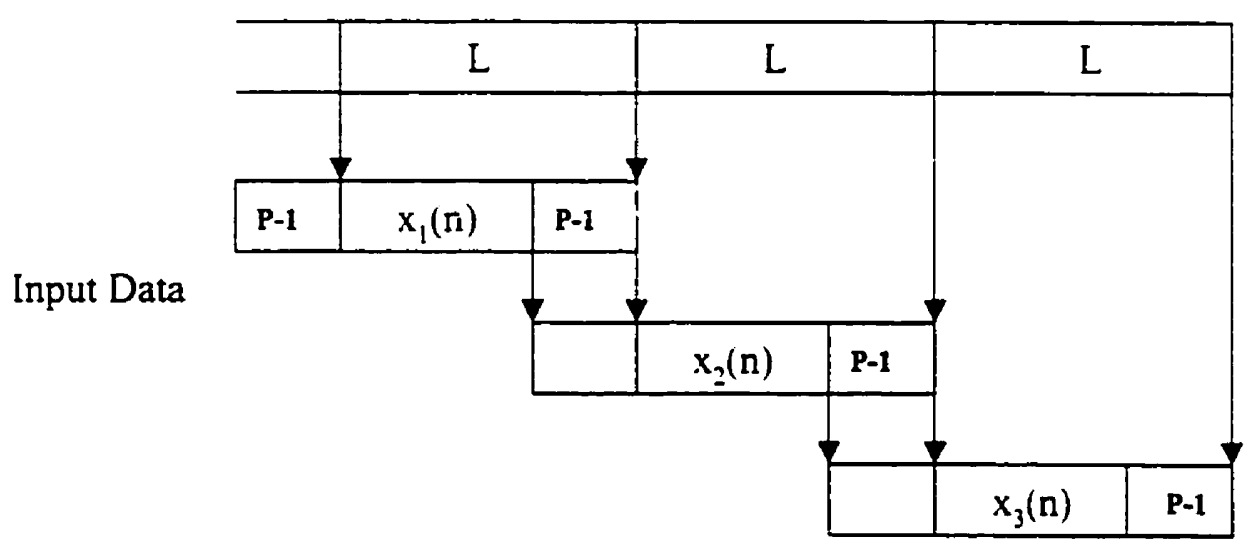

Filter
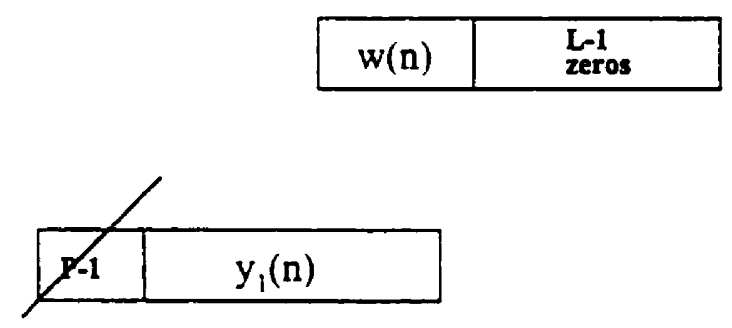

Output Data

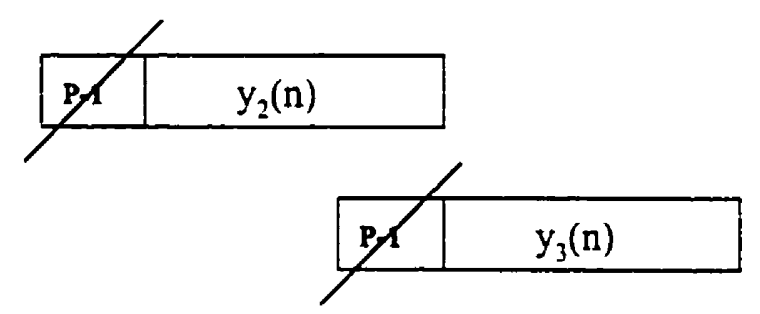

Figure 2.4: Overlap-Save Method of Linear Convolution 
preceding section with $L=2 . Y^{\circ}$ and $P=. Y-1$, where.$Y$ is the length of the equalizer. Choosing the data block size to be equal to the number of equalizer taps is known to minimize the computational complexity of the FBL.IS algorithm 15: The block diagram of the algorithm is shown in Figure 2.j. The datat is broken down into blocks of length $L=2.1$. consisting of .I samples from the previous block followed by .1 new ones. The resulting samples can be expressed as a diagonal matrix

$$
\mathbf{X}_{\mathcal{F}}(k)=\operatorname{diag}\{\mathcal{F}\{r(k . V-. Y) \ldots \ldots r(k . V-1) . r(k . V) \ldots \ldots x(k . V+. Y-1)\}\}
$$

The DFT of the equalizer taps is a column rector of length 2.1 . as the tap ralues are angmented at the end with.$Y$ zeros. that is

$$
\mathrm{w}_{\mathcal{F}}(k)=\left\{\mathcal{F}\left\{u_{v}(n) \ldots \ldots u v-1(n) .0 \ldots . .0\right\}\right\}^{T}
$$

The output vector is the element-by-element product of the transformed sequences is given by

$$
\mathrm{y}_{\xi}(k)=\mathrm{X}_{r}(k) \mathrm{w}_{. r}(k)
$$

As discussed in the section on the orerlap-save technique the first .1 samples of the product are invalid as they are the result of circular convolution. The rector $\mathbf{y}_{\mathcal{F}}(k)$ must be transformed into the time domain. where the invalid samples are discarded and only the last $X$ are used to compute the error

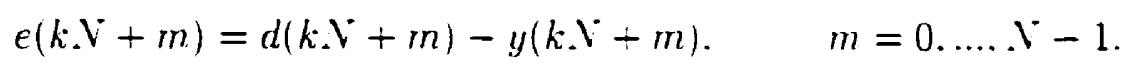

The above constraint can be formulated using the following matrix notation. By defining a sectioning constraint matrix for the error block as

$$
\mathrm{K}=\left[\begin{array}{ll}
0_{x} & I_{x}
\end{array}\right]
$$




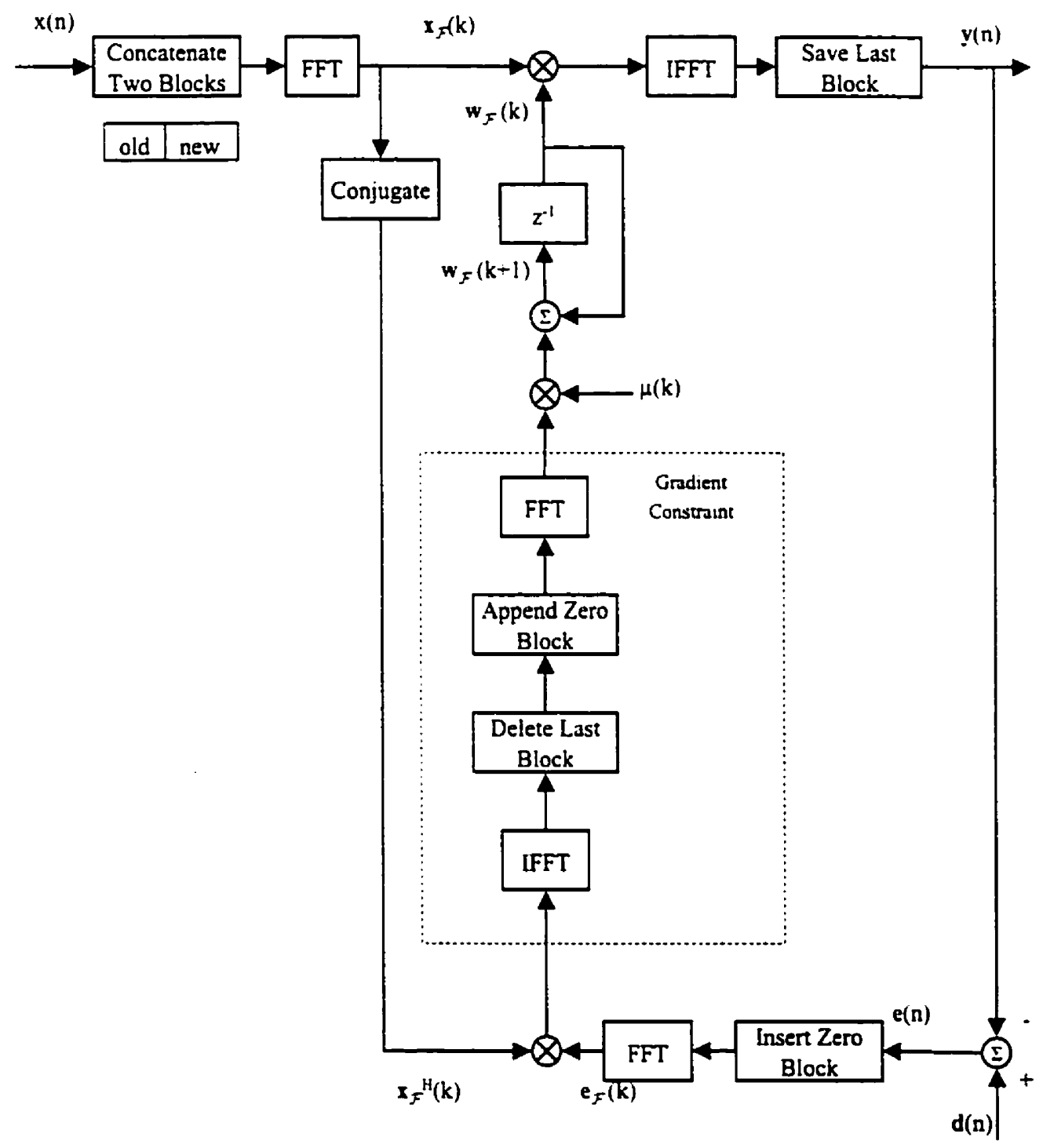

Figure 2.5: FBL.MS Algorithm L'sing Orerlap-Save Sectioning 


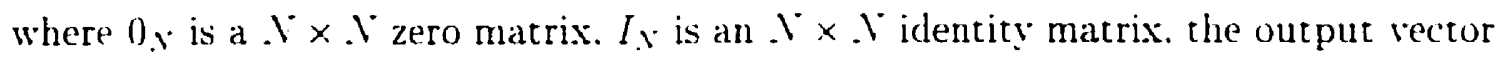
can be expressed as

$$
\mathbf{y}(n)=\mathrm{KF}^{-1} \mathrm{X}_{-}(k) \mathbf{w}_{f}(k)
$$

where $\mathcal{F}$ is a $2.1 \times 2.1$ DFT matrix with elements $\mathcal{F}_{\imath \jmath}=c^{-j 2 \pi t \jmath} 2 . x$. The resulting error samples are augmented with .1 zeros

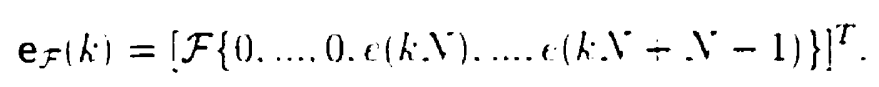

The zeros are inserted due to the fact that linear correlation has to be computed between the error vector and the complex conjugate of the input vector $\mathbf{X}_{\tilde{F}}^{H}(k)$. Ling the constraint matrix. vector $\mathrm{e}_{F}(k)$ can be rewritten as

$$
\mathrm{e}_{\mathcal{F}}(k)=\mathcal{F} \mathbf{K}^{T} \mathrm{e}(k)
$$

The next section in the flow chart is the gradient constraint. Since there are only . $V$ tap weights being updated in the time domain. it is necessary to ensure that only .7 elements of the gradient are non-zero. Thus. the gradient is transformed into the time domain and the last .7 elements are replaced with zeros before transforming it back to frequency domain and updating the tap ralues. This also means that initially the last $\lambda$ equalizer taps must be set to zero.

The gradient constraint can be expressed by using a constraint matrix similar to that used for the error block. By defining $P$ as

$$
\mathbf{P}=\left[\begin{array}{ll}
I_{x} & 0_{x} \\
0 . x & 0_{x}
\end{array}\right]
$$

the augmented gradient can be expressed as

$$
[\dot{\nabla} \mathbf{J}(k) .0 \ldots . .0]^{T}=\mathbf{P} \mathcal{F}^{-1} \mathbf{X}_{\mathcal{F}}^{H}(k) \mathbf{e}_{\mathcal{F}}(k)
$$


The tap update therefore becomes

$$
\mathrm{w}_{\Sigma}(k-1)=\mathrm{w}_{\varepsilon}(k)-2 \mu(k) \mathcal{F} \mathcal{F}^{-1} \mathrm{X}_{\Sigma}^{H}(k) \mathbf{e}_{\mathcal{F}}(k)
$$

If the step size $\mu(k)$ set to a constant. the resulting algorithm is the nun-normalized FBL.IS. However. as described in Section 2.4. the convergence properties can be improved by making the step size $\mu(k)$ inversely proportional to the power content in each frequency bin. that is

$$
\mu(k)=\mu \hat{\mathbf{D}}^{-1} .
$$

where $\mathbf{D}$ is computed similarly as in the L.IS algorithm. The resulting algorithm is referred to as the normalized FBL.IS or NFBL.MS.

In the case of both FBL.IS and NFBL.IS algorithms. the gradient constraint introduces two additional DFT operations. which significantly increases the computational load. In some applications. this constraint is dropped in order to decrease the complexity: This is referred to as the unconstrained FBL.MS algorithm. In such cases. the gradient vector no longer contains .1 non-zero entries. and wrap-around effects are introduced effecting the performance of the adaptive algorithm. This results in an increased steady state MSE and the final taps do not correspond to the ideal Wiener solution. The unconstrained algorithm can be obtained from Figure 2.5 by simply removing the dotted section labeled as 'Gradient Constraint".

\section{Misadjustment of the FBLMS and NFBLMS algorithms}

The relationship between the steady state misadjustment value and the adaptation step size for the FBL.IS and NFBL.MS algorithms has been derived in [15]. In the case of the non-normalized FBL.IS algorithm. the misadjustment of $10 \%$ or less can 
be expressed as

$$
\mathcal{M}_{F B L M S}=\mu-V_{p_{0}}
$$

where $\rho_{0}$ is the diagonal element of the correlation matrix $\mathbf{R}$. For the normalized counterpart the expression is given by [15]

$$
M_{Y F B L M S S}=\mu . V / N^{\prime \prime}
$$

where $N^{-1}$ is the total size of the overlapped input vector. usually chosen to be $2 . V^{\circ}$.

\subsubsection{Computational Complexity of the FBLMS algorithm}

The real advantage of using the FBL.MS algorithm is the savings in the computational complexity. Recall that the L.IS and the BL.MS algorithms require $2.1 \%$ real multiplications per input block. while the TDL.IS algorithm. due to the need to compute the transform after each input sample requires $2.1^{3.3}$ multiplications.

The Fast Block L.MS algorithm with the gradient constraint requires a total of $j$ FFT's of length 2.1. Assuming the algorithm uses a radix-2 decimation in time, the total number of real multiplications for a transform of block $\mathcal{V}$ of complex data is $2.2 \log .1$. For real input. the computational load decreases two-fold due to symmetry [21]. thus requiring only $. \log . \mathrm{m}$ multiplications. In addition. the FBL.MS algorithm requires 2.1 complex multiplications for computing the gradient as well as for updating the equalizer taps. The total number of real multiplications required to process a block of $\mathrm{V}$ data is $10 \mathrm{~N} \log 2 \mathrm{~V}+16 . \mathrm{V}$ and $20 . \mathrm{V} \log 2 \mathrm{~N}+16 \mathrm{~V}$ for real and complex signals respectively.

Furthermore. the step-normalized FBL.MS algorithm requires an additional 2:V multiplications of real power estimate by a complex gradient. Thus the complexity 


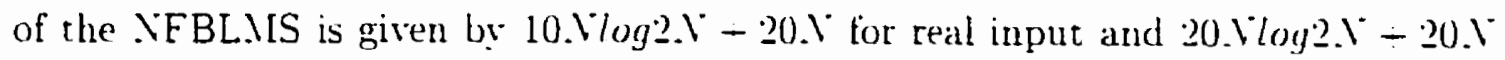
for complex input. 


\section{Chapter 3}

\section{Performance over Low Rate Channels}

\subsection{Introduction}

Performance analysis of the time and frequency domain equalization as applied to low diata rate multipath channels is discussed here. First. an overview of the communication svstem model used is given. including description of modulation methods. pulse shaping techniques and channel impulse response representation. Details of the simulations performed are described. following which we present the results. The performance criteria considered are the bit error rate. convergence properties and the computational complexity of the algorithms. Also included is a discussion of the Fourier Series technique of computing the BER for channels corrupted with additive Gaussian noise.

\subsection{Communication System Model}

The communication system model used consists of a cascade of a data source. transmit filter. channel. receive filter. equalizer. a decision device and a data sink. This is depicted in Figure 3.1. 


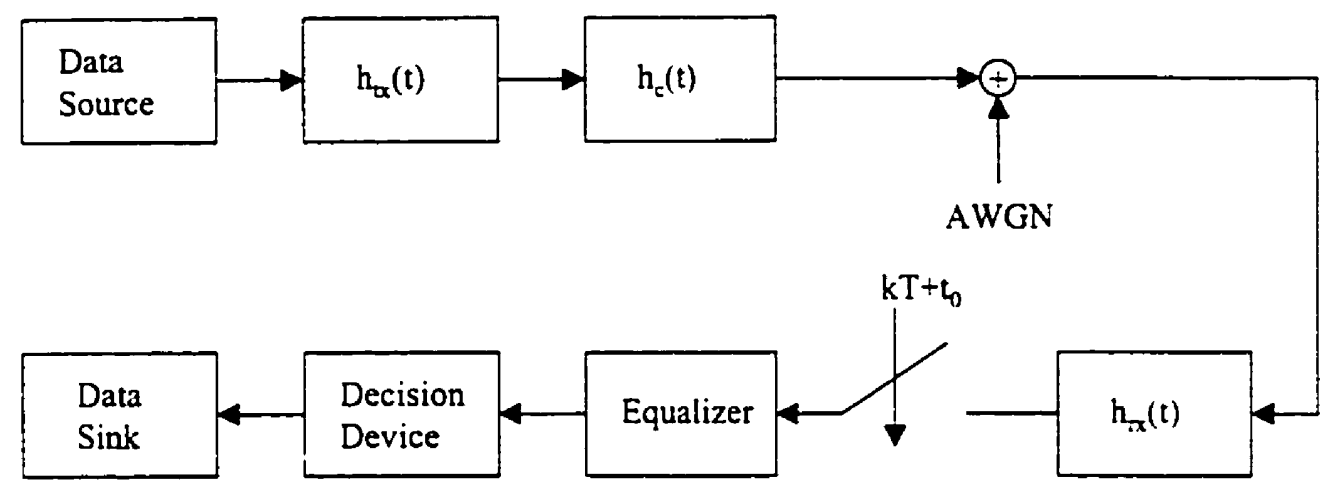

Figure 3.1: Communication Sistem . Model

The data source generates a stream of bits to be transmitted over the channel. It is assumed that the source translates the bits onto a sequence of symbols depending on the type of signaling used. These stmbols. denored as $I_{k}$. are applied to a transmit filter which generates a pulse to be transmitted over the channel. The impulse response of the transmit filter is denoted as $h_{t, x}(t)$ where $t x$ stands for transmitter. The channel. with an impulse response $h_{r}(t)$. introduces intersymbol interference (ISI) and corrupts the signal with additive white Gaussian noise (AWG.') before the input to the receive filter. The impulse response of the receive filter is denoted by $h_{r s}(t)$. where $r x$ stands for receiver. The signal at the receive filter output is sampled prior to the equalizer. In Figure $3.1 t_{0}$ is the sampling time offset for best symbol detection. The output of the equalizer is fed to the decision device which generates a sequence of detected symbols denoted by $\tilde{I}_{k}$. These sumbols are then mapped back to a stream of bits and sent to the sink.

The-svstem model described in Figure 3.1 represents a baseband equivalent of a passband system. In most real applications. in order to transmit the data over a channel. the transmitted signal spectrum is shifted onto a passband centered around a suitable carrier frequency. The process of modulation is accomplished at the trans- 
mitter by multiplying the signal by a sinusoidal carrier. Demodulating such signal requires shifting the passband signal back onto baseband by multiplying it by a replical of the carrier. In this thesis it is assumed that ideal coherent demodulation is performed. that is the carrier used at the receiver is in phase with that at the transmitter. With this assumption. the baseband equiralent model of a passband system is justified.

\subsubsection{Signaling Methods}

The input to the transmit filter is an impulse train of symbols as generated by the source. given by

$$
I(t)=\sum_{k=-\infty}^{\infty} I_{k} \dot{\delta}(t-k T) .
$$

where $\delta(t-k T)$ is the unit impulse function and $T$ is the symbol interval. To optimize transmission in a bit error rate sense. mapping of the bits onto the symbol alphabet is chosen such that the Euclidean distance berween the symbols $I_{k}$ is maximized for a given average power constraint [18]. The simplest method of accomplishing this is antipodal signaling. where the symbol alphabet is $\{-1,1\}$. This is used in conjunction with binary phase shift keying modulation (BPSK), where the phase of the sinusoidal carrier is altered by 180 degrees depending on the polarity of the symbol. An alternative method achieves double the data rate for a given bit rate by mapping each pair of bits onto an alphabet consisting of $\{1+j,-1+j,-1-j, 1-j\}$. The modulation used with such symbol mapping is referred to as quaternary phase shift keving (QPSK). which is equivalent to transmitting two BPSK streams on orthogonal carriers. 


\subsubsection{Pulse Shaping}

The shape of the received pulse is determined br the overall impulse response of the transmit filter $h_{t x}(t)$. the channel $h_{r}(t)$ and the receive filter $h_{r x}(t)$. In order to achieve zero ISI. the receive filter must be chosen such that the overall pulse shatpe:

$$
f(t)=h_{t r}(t) * h_{r}(t) * h_{r+}(t)
$$

satisfies Nyquist's first criterion. To fulfill such a requirement. the receive filter is ideally chosen such that it is matched to the combined response of the transmitter and the channel. that is

$$
h_{r r}(t)=l \cdot(-t) .
$$

where

$$
h(t)=h_{t x}(t) \times h_{t}(t)
$$

In practice the channel characteristics may be unknown and/or changing with time. As a result. suboptimum receiver filtering is used. where the received filter impulse response is matched solely to the shape of the transmit filter. Such system would satisfy liquist's first criterion in the absence of channel induced ISI. A commonly used shape is the raised cosine ( $\mathrm{RC}$ ) pulse. given by [22]

$$
h_{R C}(t)=\frac{\sin (\pi t / T) \cos (3 \pi t / T)}{(\pi t / T)\left(1-43^{2} t^{2} / T^{2}\right)} .
$$

where 3 is the excess bandwidth factor. Pulse shaping is evenly split between the receiver and the transmitter. so that the convolution of their pulses yields (3.j). Such pulse is the root raised cosine (RRC) shape giren by [22]

$$
h_{R R C}(t)=\frac{43}{\pi \sqrt{T}} \frac{\cos ((1+3) \pi t / T)+\frac{1}{43 T} \sin ((1-3) \pi t / T)}{1-163^{2} t^{2} / T^{2}} .
$$


In practice. in order to ensure causality of the filter. a delay term must be included such that the entire pulse exists only for positive time values. In the analysis here. this delay term is set to zero. with the assumption that in a real life implementation it is non-zero. thus resulting in a realizable filter.

\subsubsection{Channel}

The pulse generated by the transmit filter passes through the channel were ISI is introduced. The primary source of ISI is multipath propagation. The presence of reffecting objects and scatterers in wireless channels results in multiple versions of incoming signal arriving from different directions and with different propagation delays [23]. Such a channel can be modeled by a sequence of appropriately delaved impulses. each representing a separate multipath component. The temporal variation of the position of objects in a wireless channel results in fading or fluctuation of amplitude of multipath components. In this thesis. howerer. the study is limited to stationary channels. where the impulse response does not vary with time.

Apart from introducing intersimbol interference. the channel is also subjected to noise. White noise. defined to have a that power spectral density over the entire spectrum. is modeled by a stochastic process. Since noise is a cumulative effect of a large number of processes. it is assumed to be Ciaussian via the Central Limit Theorem [24]. In the model here presented. noise is introduced into the sistem just prior to the receive filter.

\subsubsection{Equalizer}

To combat the ISI introduced by the channel. an equalizer is included at the receiving end of the system. The equalizers considered in this model are discrete-time and are implemented using tapped delay line adaptive filters. They are placed after the 
sampler and operate on the sampled values of the received signal. This thesis examines linear equalizers. both symbol-spaced and fractionally-spaced.

\subsection{Simulation Methodology}

In the simulations performed. the data source was implemented using a uniform random number generator which generated antipodal symbols for BPSK and symbols with phases separated by 90 degrees in the case of QPSK modulation.

The generator output was convolved with the transmit filter impulse response. The transmit filter used was the RRC shape with a roll-off value of $.3=0.35$ chosen to conform with the IS-jy digital standard [22]. The pulse was chosen to be of length $8 T$. that is. it was truncated after 4 simbol intervals at each side of the peak value. The sampling resolution for the RRC filter was $T / 8$.

The channels examined in this section are two short impulse response channels chosen such that the overall impulse response of the transmitter. channel and receiver is that described in [18]. The real valued channel. referred to as Channel $A$. was used with BPSK transmission and consisted of a main component and two intersymbol interference terms. Its response is given by

$$
h_{c}(t)=-0.45 \delta(t-1.25 T)+0 . \bar{\imath} \delta(t)+0.45 \delta(t+1.25 T) \text {. }
$$

The second channel. referred to as Channel B. used with QPSK transmission. was similar to Channel A described above. but included a phase offset

$$
h_{c}(t)=0.5756 \exp (j \pi / t) \delta(t-T)+0.815 \delta(t)+0.5756 \exp (j \pi / t) \delta(t+T) .
$$

Suboptimum receive filtering was used. with the receive filter transfer function matched to the transmit filter. In the study presented here. the simulations were 
performed using digital receive filters. Implemented as a tapped delar line. their sampling rate was varied among $T / S . T / 4$ and $T / 2$ to determine the tradeoff between receiver complexity and performance.

To eliminate ISI. the receive filter of varying complexity were coupled with $T$ spaced equalizers. Also, to examine the ability of fractionally-spaced equalizers to provide receive filtering [25]. simulations using $T / 8 . T / 4$ and $T / 2$ equalizers and no receive filters were also performed. The length of the equalizer was chosen to provide sufficient elimination of ISI. For channels with short impulse response described above. equalizers spanning 16 svmbol intervals were sufficient.

Tap adaptation was implemented in time domain using the non-block LAIS algorithm and in the frequency domain using FBL.IS. The latter included constant step size adaptation as well as normalized algorithm in which the step size was raried inversely proportional to the power content in each spectral bin. Estimation of the power distribution was done by means of $(2.31)$ with the smoothing factor $-=0.95$. Both frequency domain algorithm used the orerlap-sate technique to obtain proper data sectioning thus rielding linear convolution.

A training sequence of bits was initially transmitted. For both channels. 10 blocks were sufficient to obtain convergence of the algorithm. Since the channels considered were assumed to be stationary. subsequent training sequences were not necessary. that is. data did not have to be broken down into blocks containing an initial preamble.

\subsection{Bit Error Rate Performance}

\subsubsection{BER Simulation Method}

To evaluate the bit error rate performance. the simulations were performed for signalto-noise ratios of 5 to $30 \mathrm{~dB}$. The signal-to-noise ratio was defined at the input to the 
receiver. given by

$$
S . V R=\frac{E_{11}}{x_{11}} .
$$

where $V_{0}$ is the noise variance and $E_{b}$ is the energy per data bit computed as

$$
E_{b}=\sum_{k=-x}^{x} h_{k} h_{k} \text {. }
$$

where $h_{k}$ is the discrete combined impulse response of the transmit filter. the channel and the receive filter as defined by (3.4).

\subsubsection{Series Evaluation of BER}

In [26]. Beaulieu developed an efficient method for calculating the bit error rate for channels with both cross-talk and in-phase ISI and corrupted by additive Caussian noise. The method is based on a Fourier series expansion of the noise cumulative distribution function (cdf) and (an be extended to other noise distributions provided there exists a series representation for their colf. To compute the BER. the $T$-spaced samples of the equalized impulse response must be known. along with the noise variance at the output of the equalizer. The discrete equalized system response. $\left\{g_{k}\right\}$ is the discrete convolution of the total channel samples with the equalizer coefficients. that is

$$
y_{k}=\sum_{m=-x}^{\infty} f_{m} u_{k-m} .
$$

As derrived in [27]. the BER series for QPSh signalling is given by

$$
\begin{aligned}
& P(e)=\frac{1}{2}-\frac{2}{\pi} \sum_{m=L .3 .5 \ldots}^{M}\left\{\frac{\exp \left(\frac{-m^{2} \omega^{2}}{2}\right)}{m} \cdot \sin \left(\frac{m \omega g_{0}^{l}}{\sigma_{\Sigma}^{l}}\right)\right. \\
& \left.\left[\prod_{\substack{n=-v_{1} \\
n \neq 0}}^{v_{1}} \cos \left(\frac{m \omega g_{n}^{l}}{\sigma_{z}^{l}}\right)\right] \cdot\left[\prod_{k=-x_{i}}^{v_{2}} \cos \left(\frac{m \omega^{\prime} g_{k}^{Q}}{\sigma !}\right)\right]\right\}+\Delta .
\end{aligned}
$$


where $\dot{ }^{\prime}$ is the Fourier series frequency. $\rightarrow$ is the error term and $\nu_{1}$ and $\nu_{2}$ are. respectively: the number of postcursor and precursor intersymbol interference terms in the overall equalized response. In (3.12). $g_{1}^{l}$ is the in-phase component of the desired signal term of the overall response. while $g_{n}^{l}(n \neq 0)$ and $g_{k}^{Q}$, the cluadrature component of the overall response. constitute the ISI. The noise contribution to the BER is $\sigma_{:}^{I}$. the standard deviation of the noise samples in one component at the equalizer output. computed using Equation (3.41) of [22]. The above formulation of the BER can also be used for BPSK signaling by noting that in such cases the second product scries in (3.12) equals to 1 .

The accuracy of the series is determined ly three factors: truncation of the infinite series to $M$ terms. truncation of the ISI terms and the Fourier series period $T_{F}=$ $2 \pi / 2$. The error term. $\Delta$. in $(3.12)$ is given by [2 $i$ ]

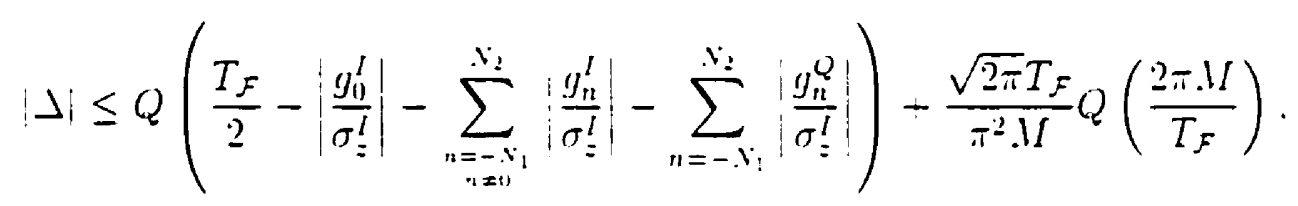

where $Q(\cdot)$ is the complementary error function

$$
Q(x)=\frac{1}{\sqrt{2 \pi}} \int_{x}^{x} \exp \left(-\frac{u^{2}}{2}\right) d u .
$$

In this thesis. all of the BER calculations were performed using $M=401$ and $T=300$. which vields results accurate to $10^{-1 \cdot 4}$ for S.R up to $30 \mathrm{~dB}[22]$.

\subsubsection{BER Results}

Figure 3.4.3 shows the bit error rate results for BPSK transmission over Channel A. Time domain L.VIS and both frequency domain block L.IS algorithms converged to the same set of equalizer taps thus yielding the same BER curves. The results obtained here are in agreement with those presented in [18]. For comparison, a 


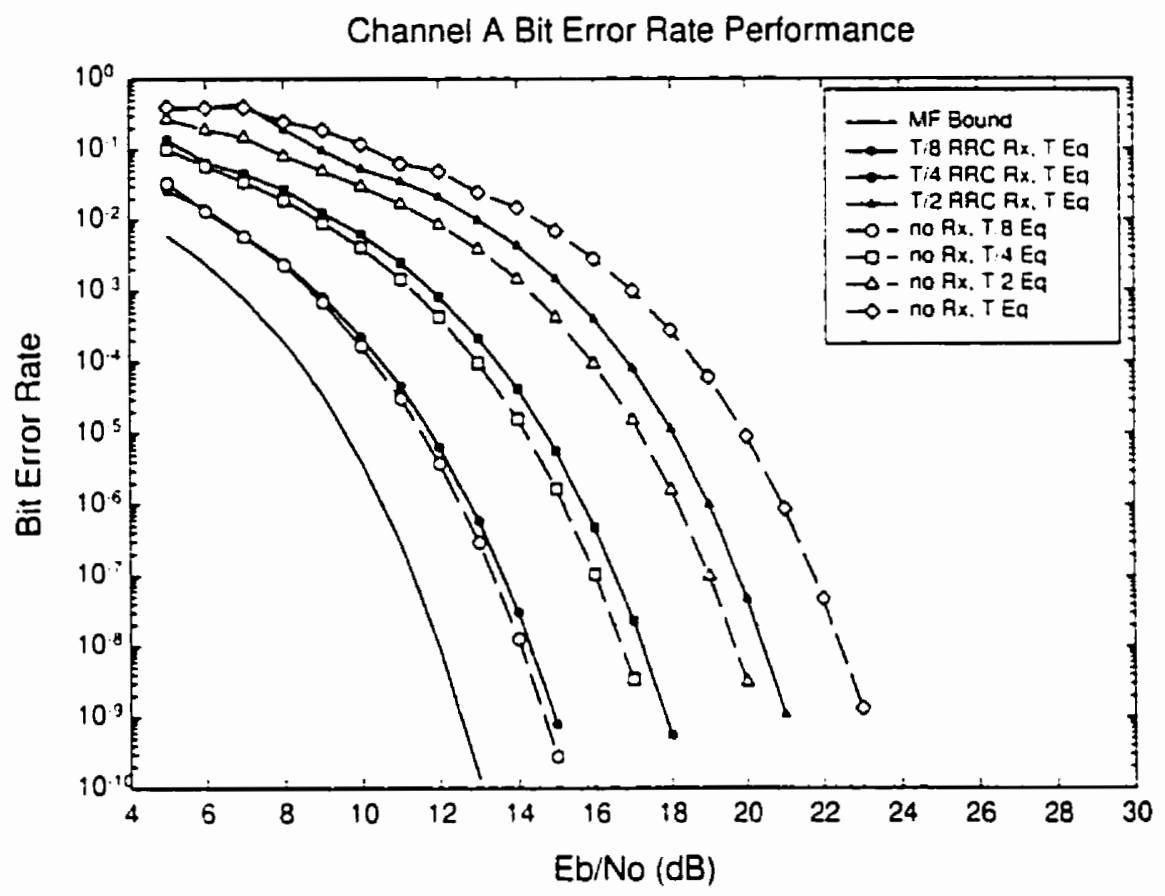

Figure 3.2: Channel A BER Results

matched filter bound. the performance achievable assuming transmission of isolated symbols (or equiralently a zero-ISI condition) [24]. is also included in Figure 3.4.3.

The $T / 8$-spaced receive filter coupled with a $T$-spaced equalizer came within 2 $\mathrm{dB}$ of the matched filter bound at $\mathrm{BER}$ of $10^{-8}$. When the complexity of the receive filter was reduced to tap spacing of $T / 4$. the performance dropped an additional 2.5 dB. Finally, another two-fold reduction in receiver spacing to $T / 2$ with a $T$-spaced equalizer resulted in a further performance drop of $3 \mathrm{~dB}$.

Removing the receive filter and increasing the complexity of the equalizer by a corresponding amount resulted in a slight performance increase over the former 
configuration. A $T /$ S-spaced equalizer without the receive filter outperformed the $T /$ S-spaced receiver coupled with a $T$-spaced equalizer by a fraction of a dB. Similarly: $T / 4$ and $T / 2$-spaced equalizers vielded better BER results in comparison to $T / 4$ and $T / 2$-spaced receivers with $T$-spaced equalizers. The reason for this slight increase in performance is that the fractionally-spaced equalizer performs a function of a matched filter as a receive filter [25]. Recall that the receive fitler is of a root raised cosine shape. that is. it is matched to the transmit filter and not to the combination of the transmitter and the channel.

Bit rrror rate performance for Channel B using QPSK signaling is shown in Figure 3.4.3. High complexity $T / 8$-spaced receiver followed by a $T$-spaced equalizer resulted in 3.5 $\mathrm{dB}$ loss at BER of $10^{-x}$ compared to the matched filter bound. A two-fold reduction in the sampling rate of the receiver resulted in an additional 2 dB loss. Finally: a receiver sampled at twice the signaling rate achieved BER of $10^{-4}$ at signalro-noise ratio of $21 \mathrm{~dB}$.

In the case of Channel B. removing the receive filter and transferring the corresponding complexity to the equalizer resulted in no performance loss.

From the results presented above. it can be seen that a fractionally-spaced equalizer is capable of effectively performing the function of a receive filter. This finding has been discussed in [25]. Later in this chapter we examine the complexity of integrating the receive filter into the equalizer.

\subsection{Convergence Properties}

This section presents a study of the convergence properties of time domain non-block L.IS and the frequency domain block L.IS algorithms as applied to low data rate channels. The learning curve of the FBL.MS algorithm was examined without step 


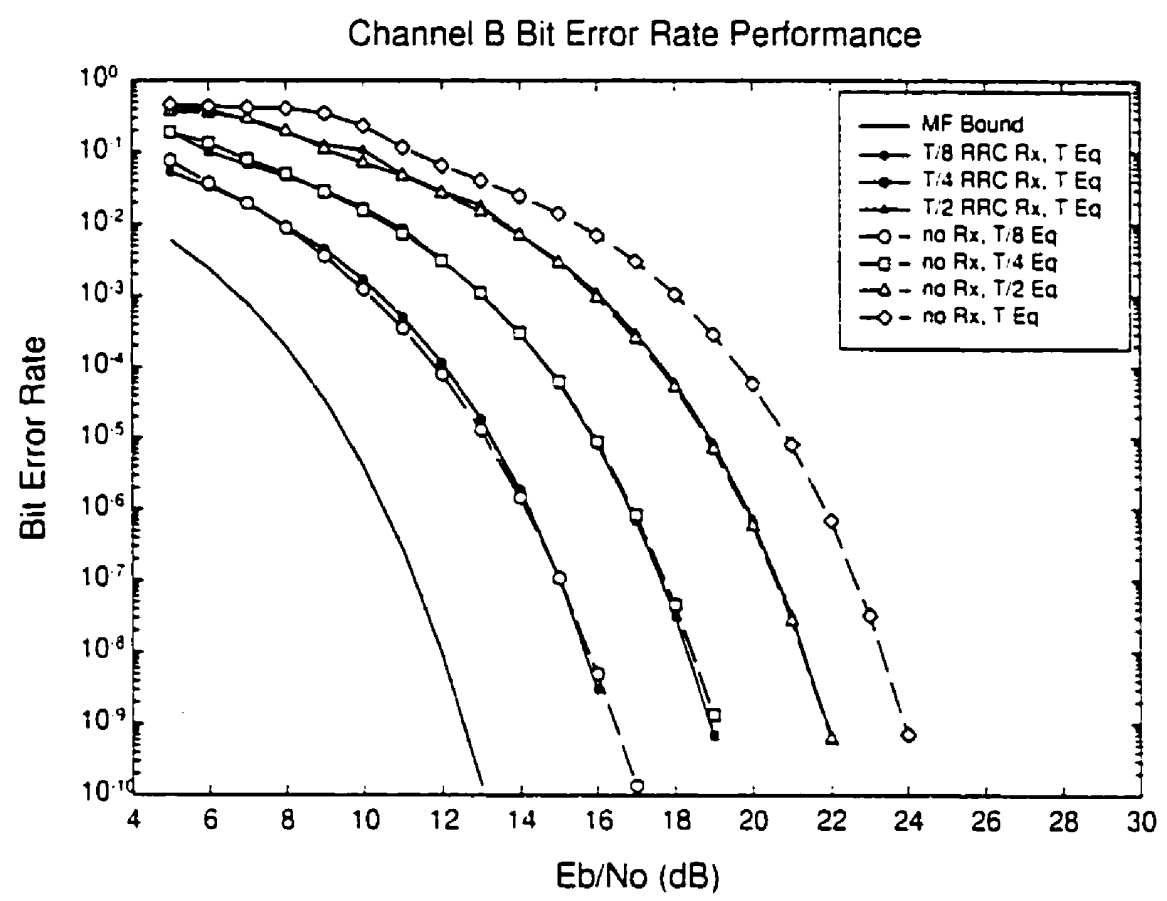

Figure 3.3: Channel B BER Results 
normalization and subsequently with step size normatized according to the power distribution across spectral bins.

Equalizers with sampling rate equal to the sumbol interval were used and were preceded by a $T /$ s-spaced receiver filter. The simulations were run orer Channels $A$ and $B$ at signal-to-noise ratio of $25 \mathrm{~dB}$. An initial training secquence of 10 data blocks was transmitted. Each simulation consisted of an ensemble of 100 independent runs. To compute the mean squared error. the squared error was obtained after wach algorithm iteration and averaged over the ensemble of tests.

In order to fairly compare the convergence properties of each algorithm. the step size was selected such as to obtain a final misadjustment value of $10 \%$ according to (2.26) (2.80) and (2.81). The step size in the tap update equation for the NFBL.IS algorithm was chosen to be inversely proportional to the power estimate in the corresponding frequency bin. This estimation was calculated iteratively based on (2.31) with the smoothing factor $=0.9 \%$. In order to increase the stability of the algorithm in the event of a weakly excited frequency: a constant value of $a=0.1$ was added. resulting in the power estimate equation given by

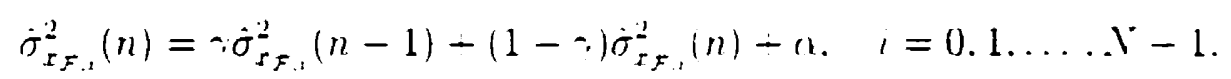

The above recursion formula for power estimate does not always vield an accurate result during the first few iterations. In order to aroid an initial spike in the learning curve due to an incorrect step size normalization. the initial estimate of the power content was computed as an average over the first 3 iterations. During these iterations the step size was held constant.

Figure 3.5 shows the learning curve of the L.IS. FBL.MS and NFBL.MS adaptation schemes for BPSK signaling orer Channel $A$. Iteration number shown on the horizontal axis refers to the number symbols processed by the equalizer, or equira- 


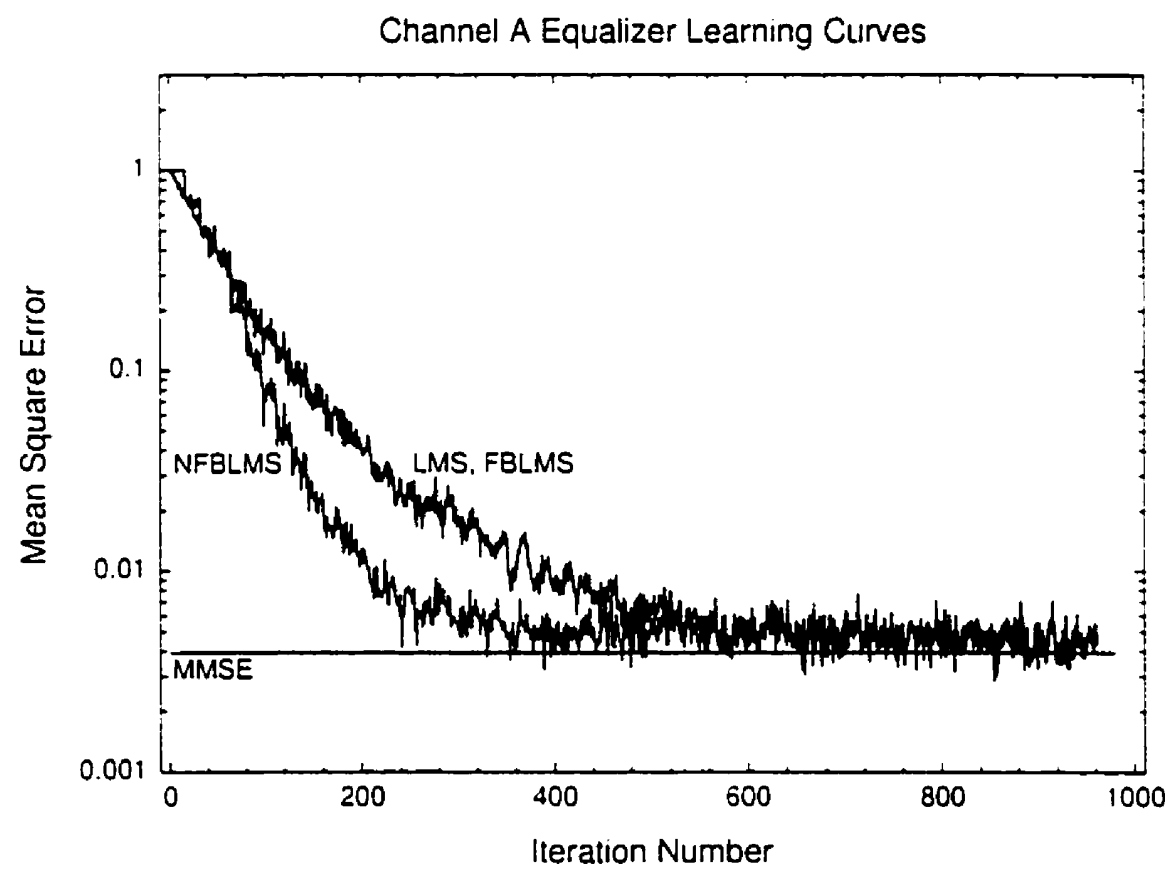

Figure 3.4: Channel A Equalizer Learning Curves

lently: the number of tap adaptations for the L.MS algorithm. As a reference point. the minimum mean square error as calculated by (2.19) is included.

As the results indicate. the LMS and FBL.MS algorithms converged at the same rate. which supports the theory presented in Chapter 2. Transforming the input data sequence to the frequency domain does not affect the shape of the performance surface. but only rotates it. Consequently the learning curves for both algorithms are nearly identical. Defining convergence point as the iteration number where the mean square error comes within approximately $5 \%$ of the steady state value. we see that both algorithms converge to near the minimum mean square error after approximately 


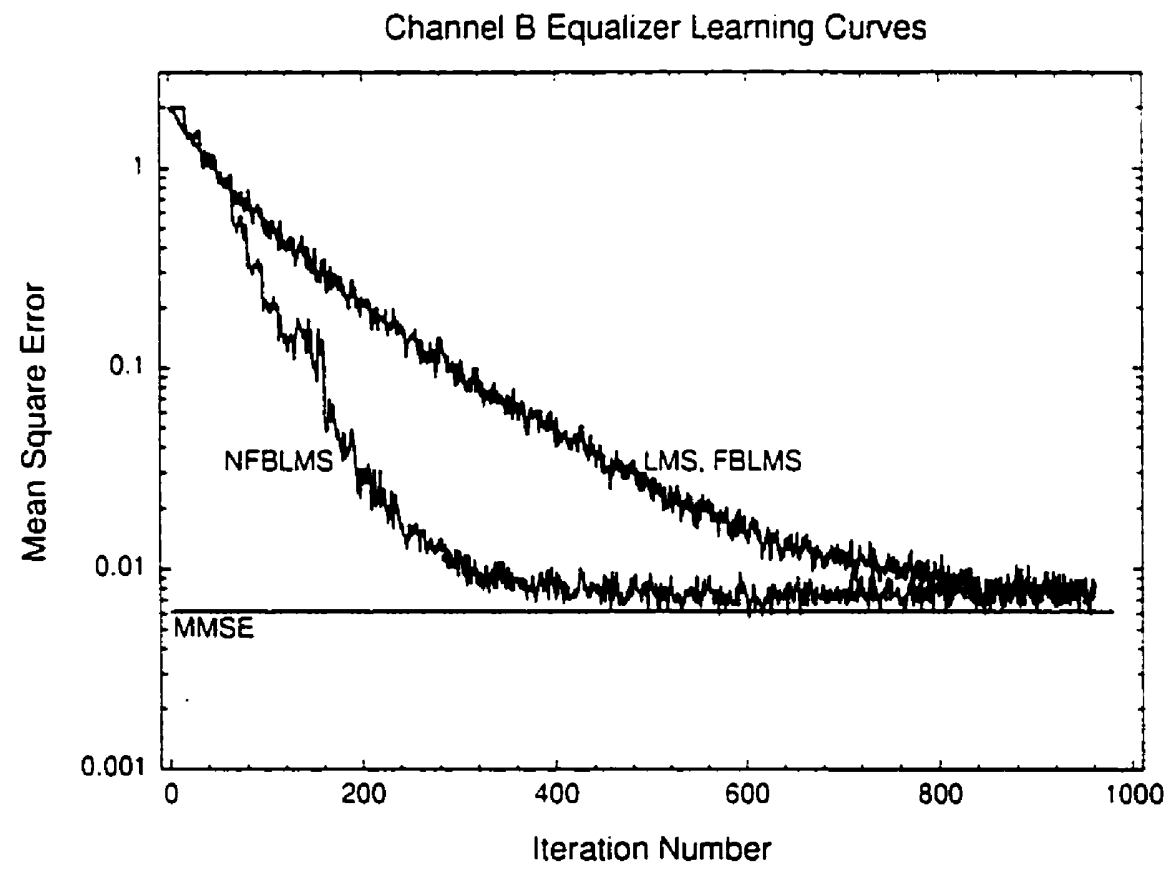

Figure 3.j: Channel B Equalizer Learning Curves 
500 iterations.

As predicted by the theory presented in Chapter 2. normalizing the step size according to the power estimate of the input signal at each frequency bin results in a significant improvement in the learning curve. When using the NFBL.MS algorithm. the steady state value of the . MSE is reached at romghly 250 iterations.

To support these findings. We now examine the eigenvalue spread of the input correlation matrix for the L.IS. FBL.MS and IFBL.IS equalizers. For the (alse of the L.MS and FBL.MS algorithms. the eigenwalue spread of the correlation matrix for Channel At can be calculated to be

$$
\frac{\lambda_{\max }}{\lambda_{\min }}=5.346
$$

In contrast. transforming the input data block into the frequency domain and normalizing the correlation matrix. results in a eigenvalue spread

$$
\frac{\lambda_{\max }}{\lambda_{\min }}=1.270
$$

which. as shown in Figure 3.j. causes more uniform convergence in each mode.

Figure 3.5 shows the learning curves of the algorithms for QPSK transmission over Channel B. Once again. the L.MS and FBL.MS algorithms exhibit nearly identical convergence properties. In the case of the L.IS and FBL.MS algorithms, the eigenvalue spread of the input correlation matrix for Channel B was calculated to be

$$
\frac{\lambda_{\max }}{\lambda_{\min }}=9.434
$$

With these eigenvalues controlling the modes of converges. the L.MS and FBL.MS algorithms reach a near steady state ISSE after approximately 800 iterations. Similarly to the findings for Channel $A$. normalizing the step size results in a significant 


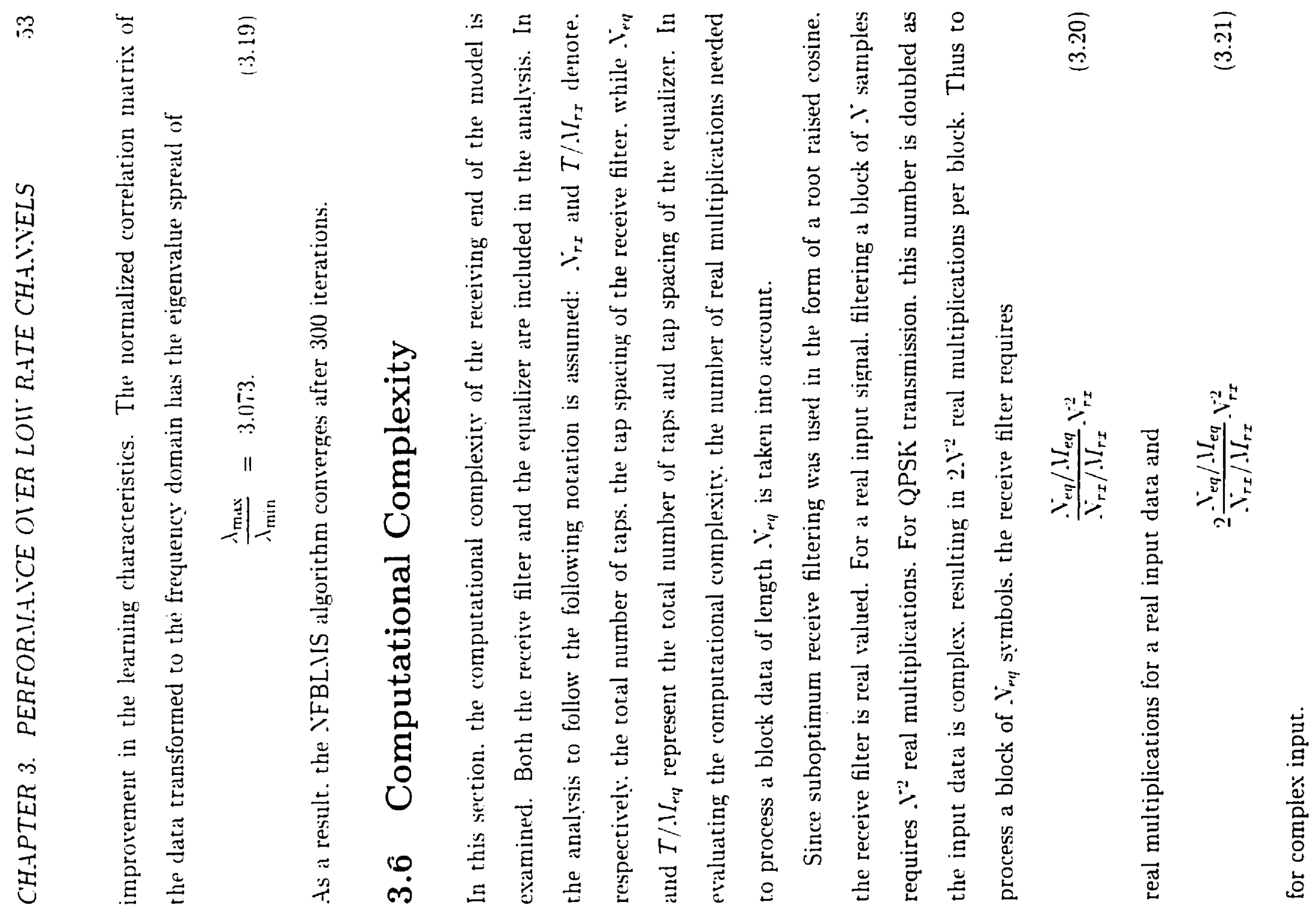


As discussed in Section 2.3.1. for the case of BPSK signaling. an adaptive equalizor implemented using the L.IS algorithm requires tutal of $2 . V_{p q}^{2}$ real multiplitations to process a block of $N_{e q}$ samples. For QPSK transmission. the computationial complexity increases by a factor of 4 . resulting in $8.1_{-1}^{\prime \prime}$ multiplications per block of data.

Computational complexity of the FBL.\S algorithm was analyzed in Section 2.6.3. Recall that a constrained algorithm requires a total of 5 FFT's of length $2 . \lambda_{* 4}^{*}$. For complex input data. each FFT can be computed using $2 . V_{e q} \log . V_{e q}$ multiplications. while for real input. the computational load decreases by half to $._{e q} \log ._{\text {eq }}$. In addition. the FBLISS algorithm requires $2 . \%_{e q}$ complex multiplications for computing the gradient and for updating the equalizer taps. The total number of real multiplications required to process a block of.$_{e q}$ data is $10 ._{e q} \log 2 . V_{e q}+16 N_{e q}$ and $20 . \Lambda_{p q} \log 2 \Lambda_{e q}-16 . \Lambda_{e q}$ for BPSK and QPSK signaling respectively. As described in Section 2.6.3. the normalized FBL.IS algorithm requires $10 . V_{e q} \log 2 . \gamma_{e q}+20 . V_{e q}$ real multiplications for real input and $20.1_{r q} \log 2 x_{r q}-20 . x_{r q}$ for complex input.

Table 3.1 summarizes the total computational complexity for a $T / M M_{r r}$-spaced receiver with a total number of taps $\lambda_{r_{S}}$ and a $T / M_{r q}$-spaced linear equalizer of total length $N_{\text {eq }}$.

Table 3.1: Complexity of the L.MS. FBL.IS and NFBL.IS Algorithms

\begin{tabular}{|c|c|c|}
\hline & $\overline{\mathrm{BPSK}}$ & QPSK \\
\hline L.IS & 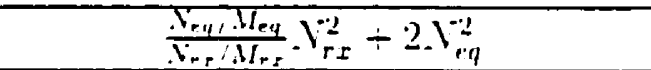 & 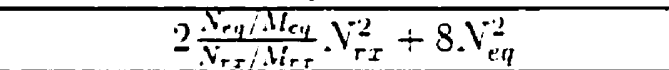 \\
\hline FBL.IS & 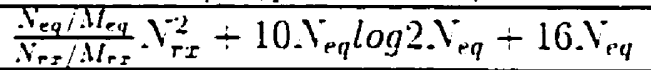 & $2 \frac{N_{e q} / . I_{e q}}{N_{r r r} / M r_{r s}} \cdot V_{r x}^{2}+20 N_{e q} l o g 2 . N_{e q}+16 N_{e q}$ \\
\hline$\therefore F B L M S$ & $\frac{V_{e q} / M I_{e q}}{V_{r z} / M_{r t}} V_{r x}^{2}+10 N_{e q} \log 2 . V_{e q}+20 V_{e q}$ & $2 \frac{N_{r q} / N_{r q}}{l_{r r r} / M_{r x}} V_{r x}^{2}+20 N_{e q} \log 2 N_{e q}+20 N_{e q}$ \\
\hline
\end{tabular}

Table 3.2 shows tabulated results for a $T / 8$ - spaced receiver spanning a duration of 8 symbols followed by a $T$-spaced equalizer of length 16 . 
Table 3.2: Complexity of a $T$ /8-spaced Receiver and a $T$-spaced Equalizer

\begin{tabular}{|c|c|c|}
\hline & BPSK & QPSK \\
\hline L.IS & 8704 & 18432 \\
\hline FBL.IS & 9248 & $18 \cdot 240$ \\
\hline JFBL.MS & 9312 & 18304 \\
\hline
\end{tabular}

The above results demonstrate that when the receive filter is followed by a lin(ar equalizer. the FBL.MS and XFBL.MS algorithms require approximately the same amount of computation to process a block of data. For BPSK signaling. the frequency domain algorithms are computationally heatier by approximately $7 \%$. while in the case of QPSK. they are less intensive by $1 \%$. However. the relatively large inprovement in convergence properties as demonstrated in Section 3.5 justifies the use of the NFBL.IS algorithm.

Remoring the receive filter from the system and increasing the tap spacing of the equalizer allows for a receiver consisting of only one structure. Complexity for an arrangement where $V_{r s}=0 . V_{r q}=16 \times 8$ and $. Y_{r q}=8$ is tabulated in Table 3.3 .

Table 3.3: Complexity of a $T / 8$-spaced Equalizer

\begin{tabular}{|c|c|c|}
\hline & BPSK & QPSK \\
\hline L.IS & 32768 & 131072 \\
\hline FBL.IS & 12288 & 22528 \\
\hline XFBL.IS & 12800 & 23040 \\
\hline
\end{tabular}

For a system with no receive filter. where the equalizer consists of fractionallyspaced taps. the L.MS algorithm requires a considerably large amount of computation. Implementing the equalizer using either the FBLISS or NFBL.MS algorithms results in a comparable computational complexity: The increase of approximately $30 \%$ can 


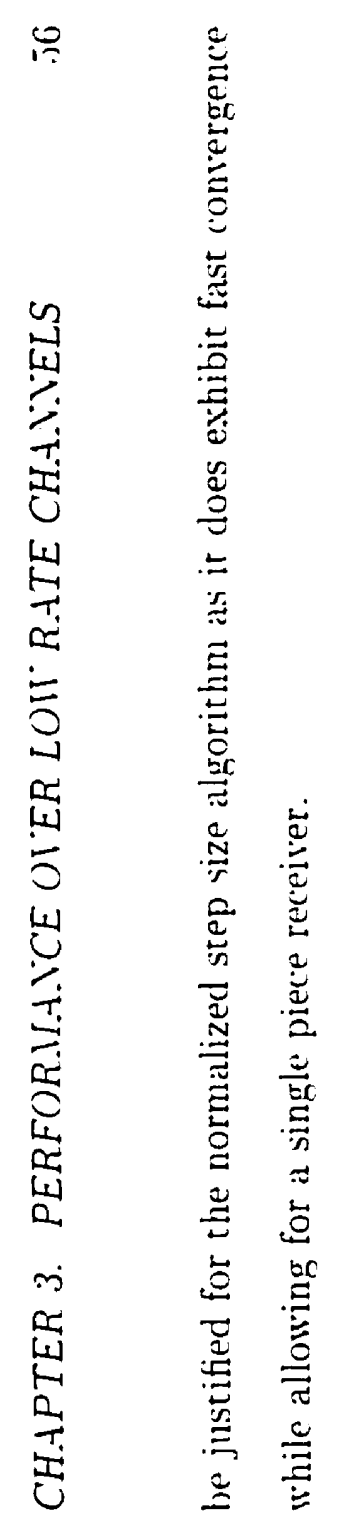




\section{Chapter 4}

\section{Performance over High Rate Channels}

\subsection{Introduction}

Simulation results for high data rate multipath channels are presented here. Since such channels are characterized by a relatively long dispersion length. the elimination of intersimbol interference requires equalizers with a significantly larger number of taps compared to that used in the case of low clata rate transmission. In such applications one expects the efficiency of frequency domain equalization to be advantageous.

The length of the equalizers emploved in high data rate systems greatly exceeds the length of the receive filter. Based on the analysis from Chapter 3. a single structure receiver consisting of a fractionally spaced equalizer is not practical from the computational efficiency point of tiew. For this reason we examine only the conventional receiver consisting of a cascade of a receive filter and a $T$-spaced equalizer.

Studies were performed on two channels. referred to here as Channel $\mathrm{C}$ and Channel $D$. characterized by long delay spreads often encountered in urban environments [28]. Channel C. whose delay profile is shown in Figure 4.1. represents a high rate channel with a maximum delay spread of $8 \mu s$. Reference [29] conducts a statistical 


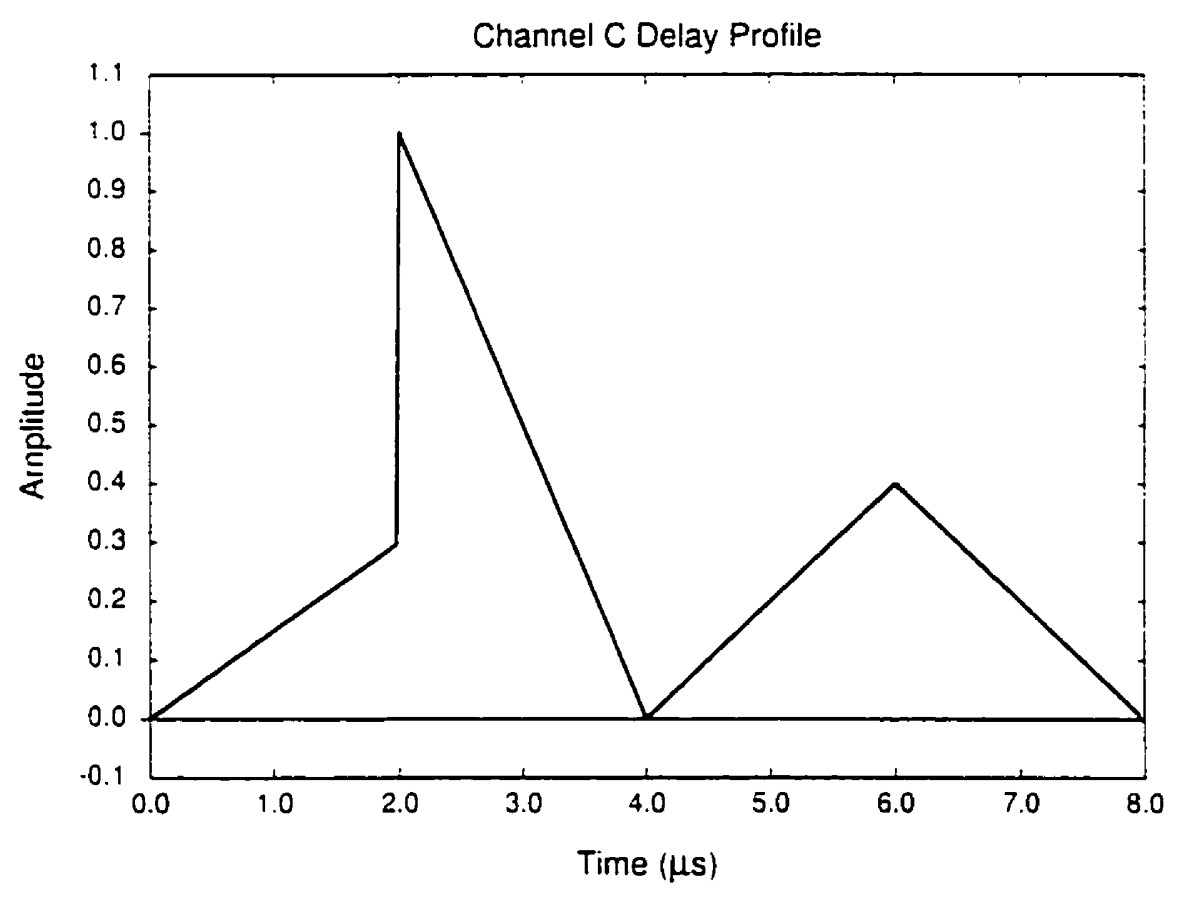

Figure 4.1: Channel C Delay Profile

analysis of wireless channel impulse responses at $1900 \mathrm{MHz}$. The shape of Channel D. whose delay profile is shown in Figure 4.2. was chosen based on the analysis performed by [29] in several Quebec City locations. The maximum delay spread of Channel $\mathrm{D}$ is $5 \mu$ s. Both channels were modeled by uncorrelated $T / 8$-spaced multipath components. The impuise response was obtained by varying the phase according to a uniform distribution from 0 to $2 \pi$ and held constant for every symbol. Typical frequency spectra of Channels $C$ and $\mathrm{D}$ are shown in Figures 4.3 through 4.5. The spectrum for Channel $D$ was quite constant relative to the choice of the random phase per stmbol. However. in the case of Channel $C$. the spectrum varied with the phase 


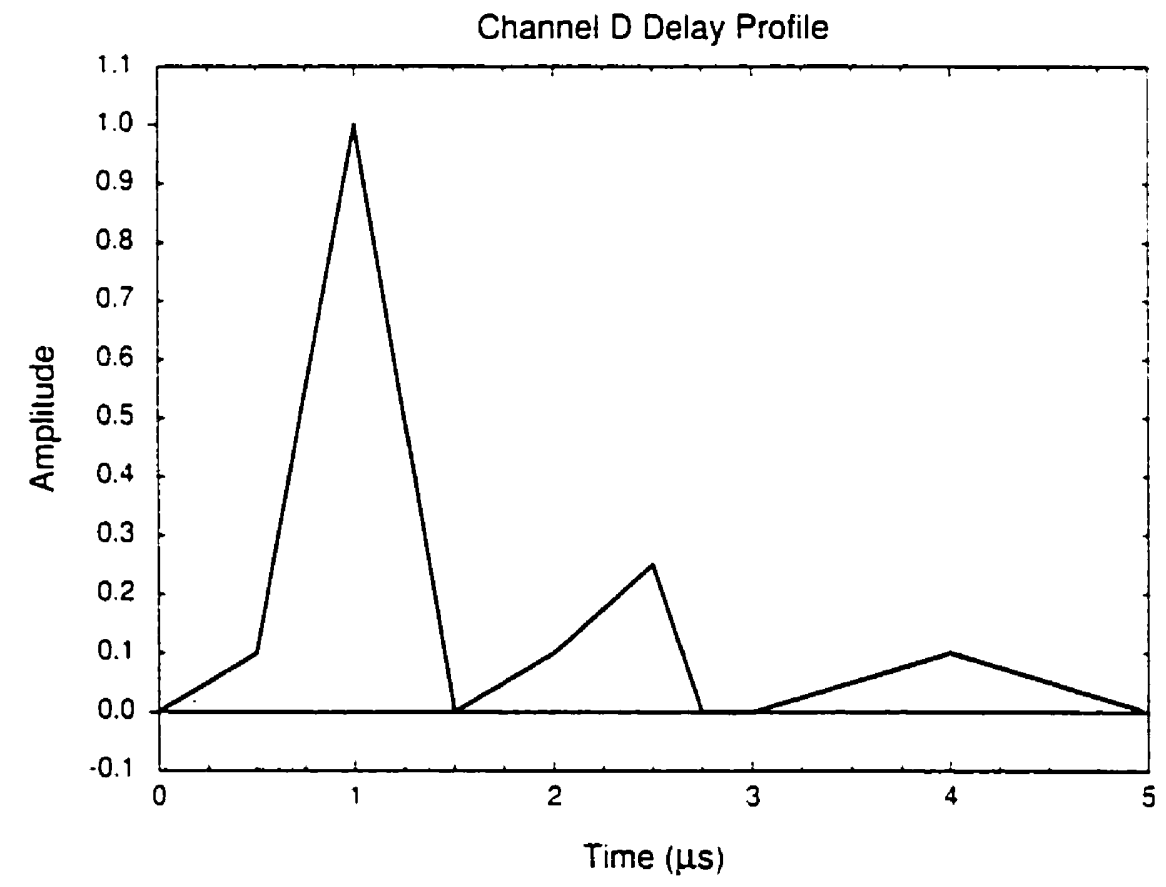

Figure 4.2: Channel D Delay Profile 


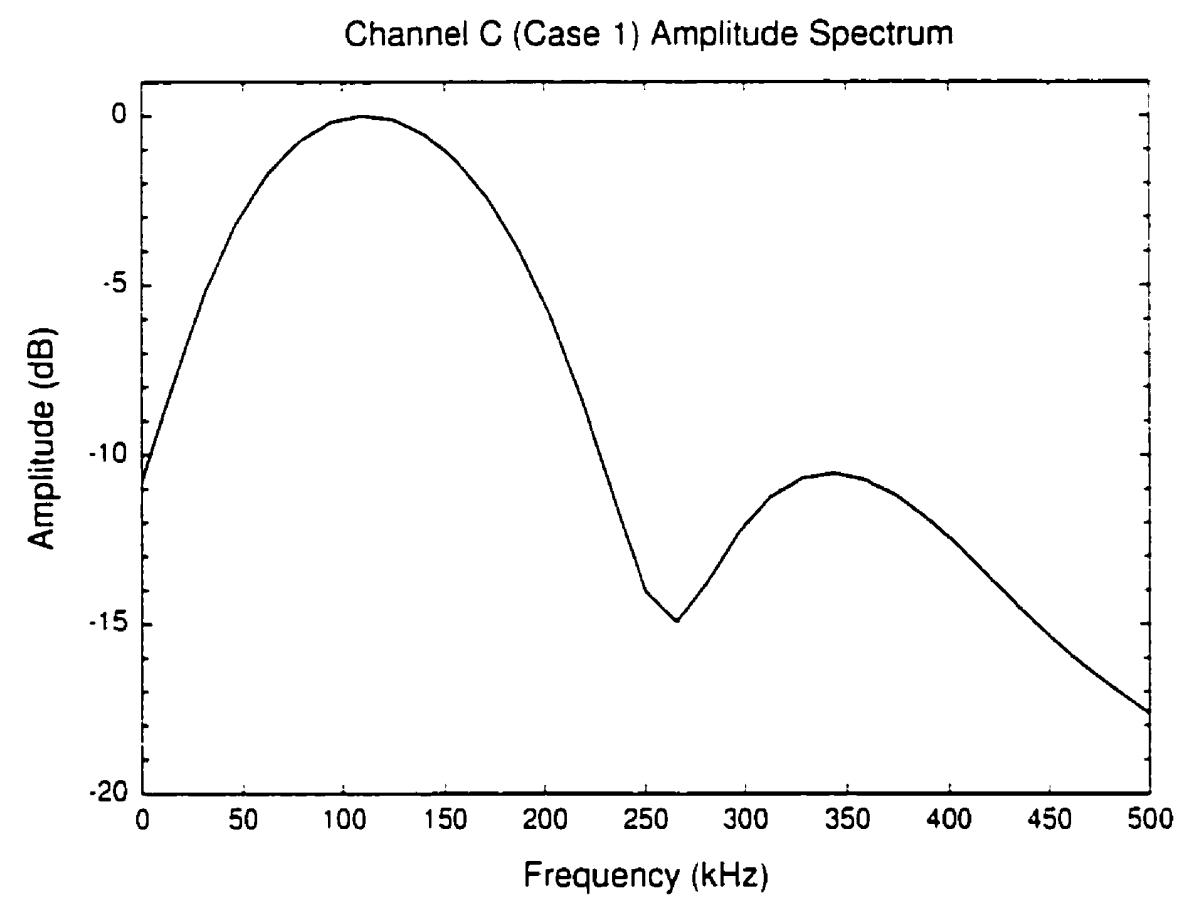

Figure 4.3: Channel C (Case 1) Amplitude Spectrum

samples. For instance. from Figure 4.3 we see that the channel contains a an inband null at DC. Such nulls are known to result in poor performance in the case of a linear equalizer [18][25]. In order to examine the effects of the null on the equalizer performance. a second instance of Channel C. one with a different phase characteristic. was also included in the BER analysis. The spectrum for case 2 of Channel C. which does not contain an inband null. is shown in Figure 4.4 . Assuming that the bandwidth of a channel is defined by the frequency at an amplitude drop of $5 \mathrm{~dB}$ relative to 0 dB. the bandwidth of Channel $\mathrm{C}$ for cases 1 and 2 is approximately $200 \mathrm{kHz}$ and 100 kHz. respectively: Channel D. with a shorter delay profile. has a larger bandwidth of 


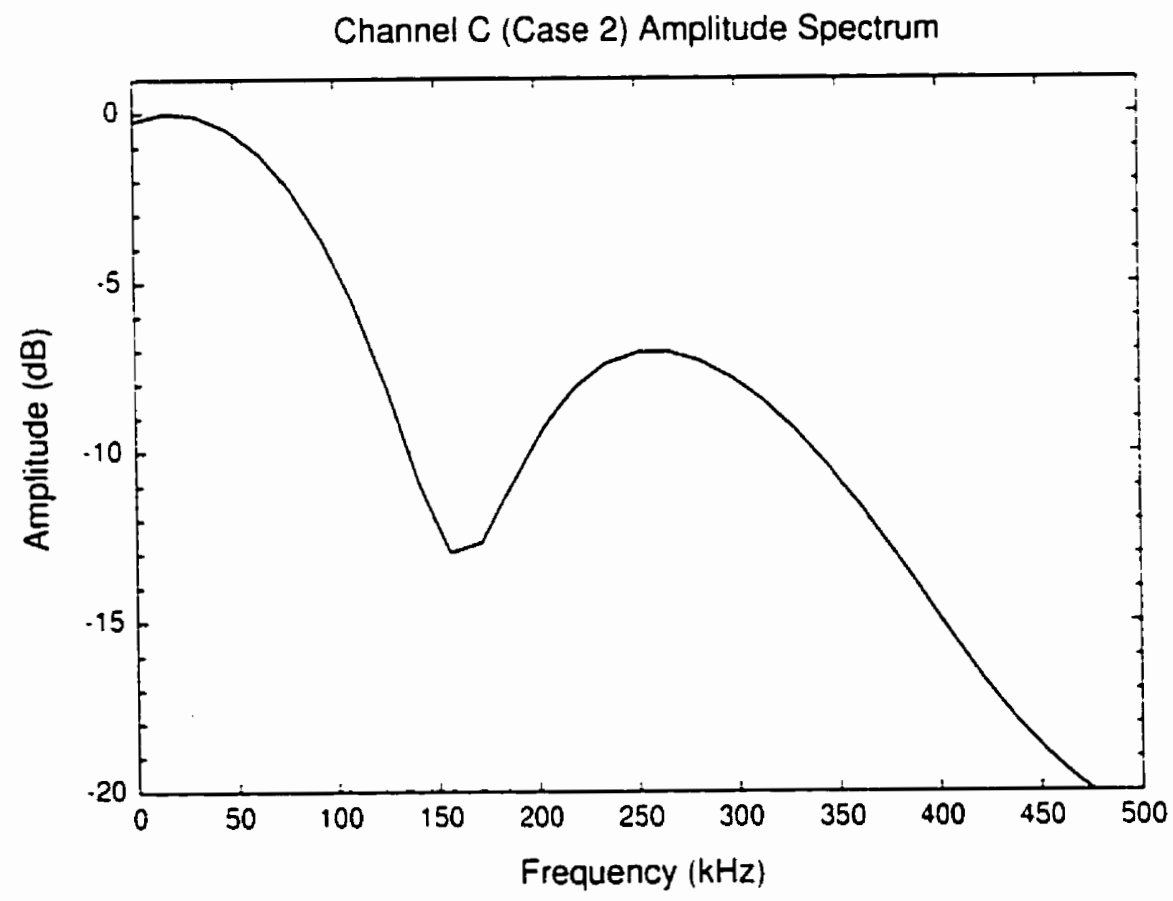

Figure 4.4: Channel C (Case 2) Amplitude Spectrum 


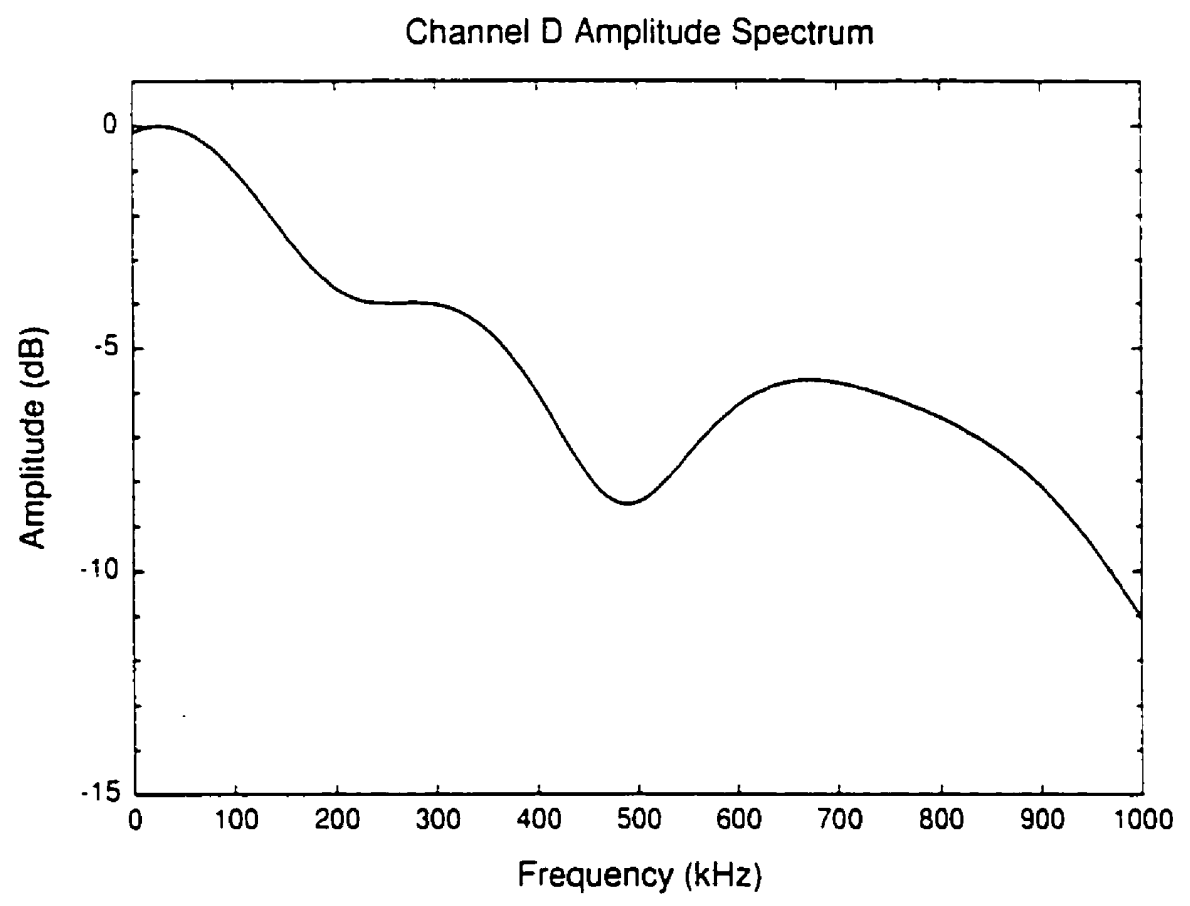

Figure 4.5: Channel D Amplitude Spectrum 
$400 \mathrm{kHz}$. The simulations were performed using BPSK and QPSK transmission. The data rates were varied from 0.1 to 2. Mbps for BPSK and 0.2 to 4 .Mbps for QPSK. We see that since these data rates exceed the Arquist rate defined as twice the coherence bandwidth of the channel. such transmission results in significant distortion [23] 30 !.

The receive filter used wass of the root raised cosine shape sampled at at rate of $T / S$ and spanning 8 symbols. We note that the timing phase at the detector was optimized in give the lowest BER in all the examples to follow. The choice of the sampling phase was done on a per-channel basis. The signal-to-noise ratio $E_{b} / N_{0}$ wass computed as given by (3.9) and (3.10). Fur data rates of 0.1 to 1 Mlbps BPSK (0.2 to 2 . Mbps for QPSK). the receive filter was followed by a $T$-spaced linear equalizer of length 32. For higher rates. the number of taps was increased to 64 .

The chapter is divided as follows. First. the bit error rate performance for both channels is examined for BPSK and QPSK modulation methods. Following the BER results. we present the convergence characteristics of the L.MS. FBL.IS and NFBL.MS algorithms. Adaptation learning curves are shown along with the analysis of the eigenvalue spreads of input data correlation matrices. To support the experimental results. we present an analysis of the input data correlation matrix for long equalizer responses. We show that in such cases [9][31][32]. the correlation matrix is approximately circular and thus can be diagonalized by the discrete Fourier transform. This property results in a vastly improved convergence rate of the frequency domain algorithm. provided step size normalization is performed. Finally in the last section. the computational complexity for the L.IS. FBL.IS and NFBL.IS algorithms is compared. 


\subsection{Bit Error Rate Performance}

The bit error rate (BER) was computed using the series technique described in Section 3.4.2. The L.IS. FBL.MS and NFBL.IS adaptation algorithms produced nearly identical sets of equalizer taps thus vielding the same BER performance. The BER results presented here are those corresponding to equalizer taps calculated using the NFBL.MS algorithm.

\subsubsection{BER Performance as a Function of SNR}

Figures 4.6 and 4.7 show the BPSK and QPSK bit error rate results for case 1 of Channel $C$ as a function of the signal-to-noise ratio (S.MR) as defined by (3.9). In the case of BPSK signaling over Channel $C$. in order to achieve a bit error rate' of $10^{-4}$ for 0.5 .Mbps BPSK (1.0 Mlbps QPSK), a signal-to-noise ratio of approximately $11.5 \mathrm{~dB}$ is required. In order to maintain a steady bit error rate. the signal power must be increased by approximately $4 \mathrm{~dB}$ for each $0.5 \mathrm{Mbps}$ increase in transmission rate between 0.5 and $1.5 \mathrm{Mbps}$. The performance drop at $\mathrm{BER}$ of $10^{-4}$ between 1.5 and 2.0 Mbps is approximately 1.5 dB. A similar trend occurs for QPSK modulation where the same increase in S.MR is needed for each $1.0 \mathrm{Mbps}$ increase between 1.0 and 3.0 Mbps. Once again the performance drop between 3.0 and 4.0 Mbps is on the order of $1.5 \mathrm{~dB}$.

Bit Error Rate results for case 2 of Channel $C$ are shown in Figures 4.8 and 4.9 . We see that the bit error probability at data rates of 0.5 .1 .0 and 2.0 .lbps BPSK is nearly identical to that for case 1. The difference occurs for 1.5 Mbps BPSK where the performance it comparison to case 1 drops by approximately $1 \mathrm{~dB}$. We see that despite the lack of an inband null. the BER performance for case 2 does not improve. This may be attributed to the fact that the bandwidth of the channel in case 2 is 


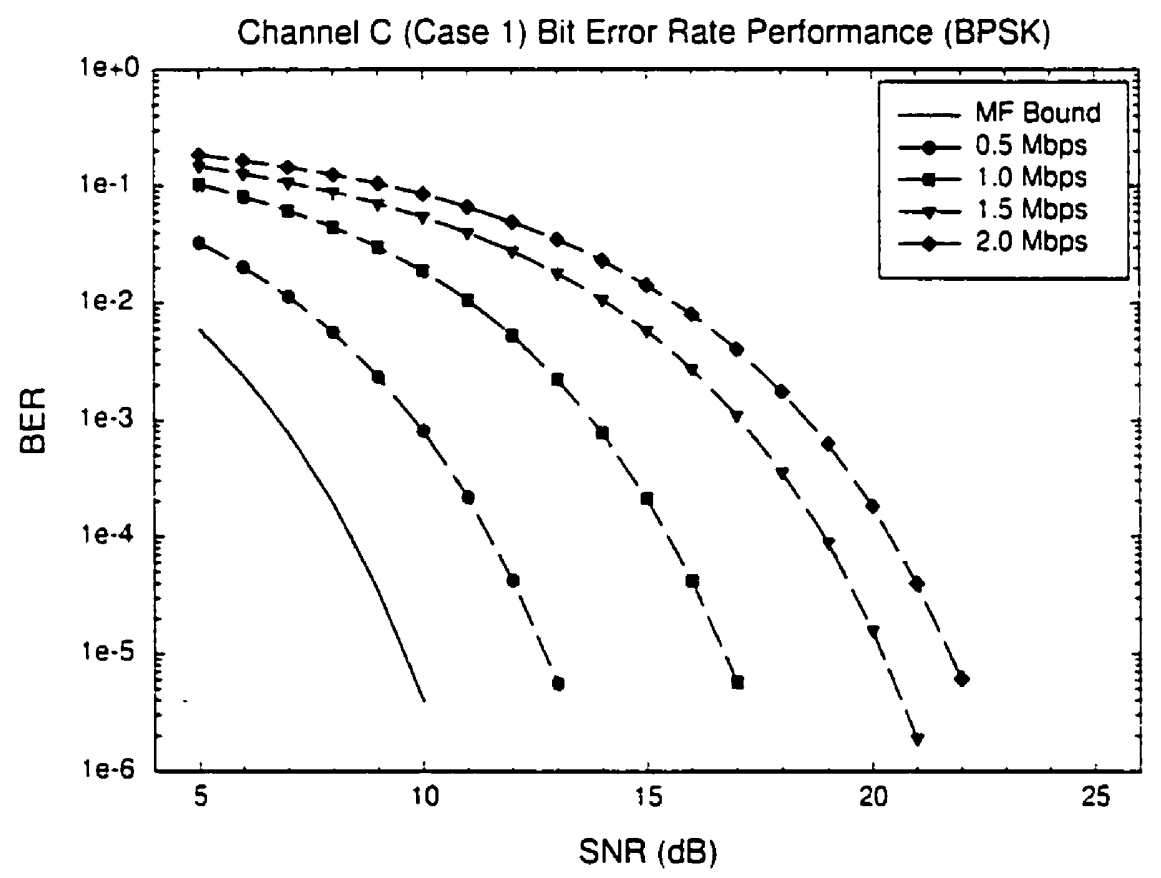

Figure 4.6: Channel C (Case 1) BER. Results (BPSK) 


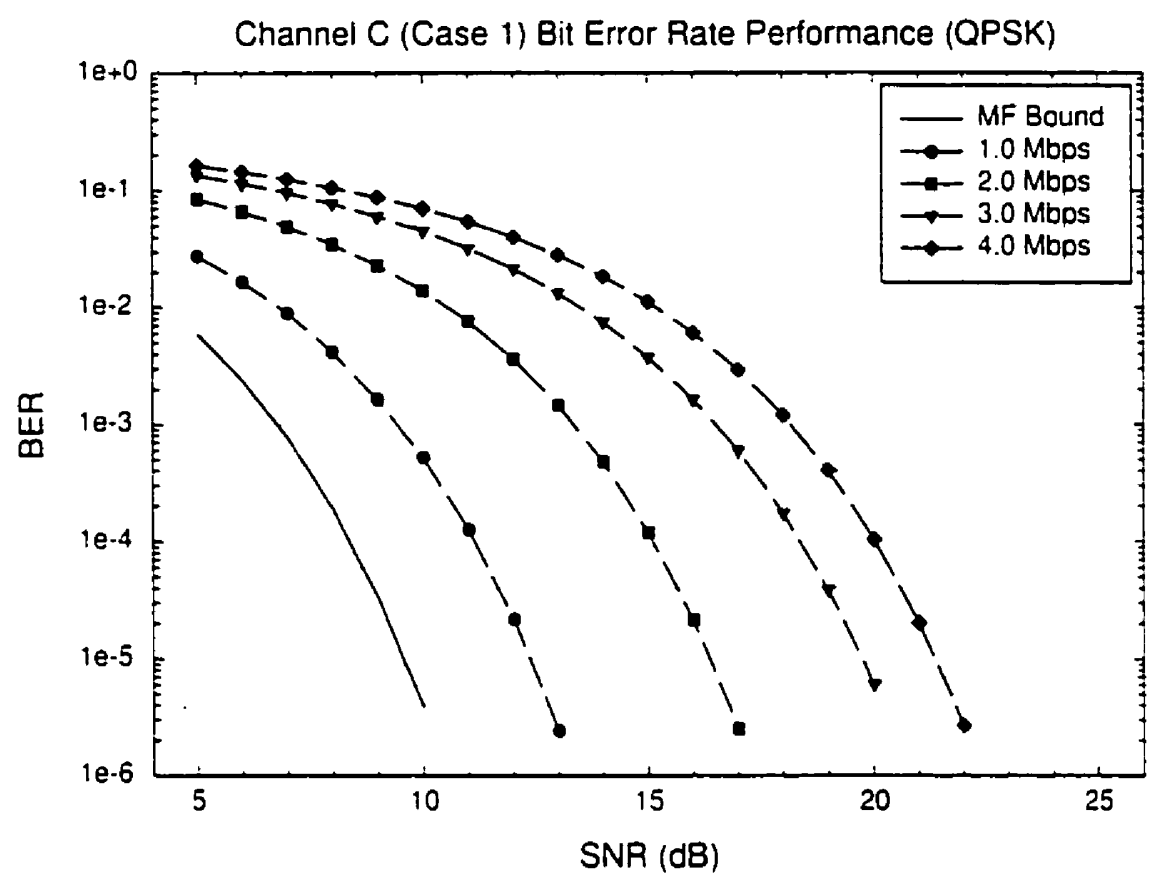

Figure 4.i: Channel C (Case 1) BER Results (QPSK) 


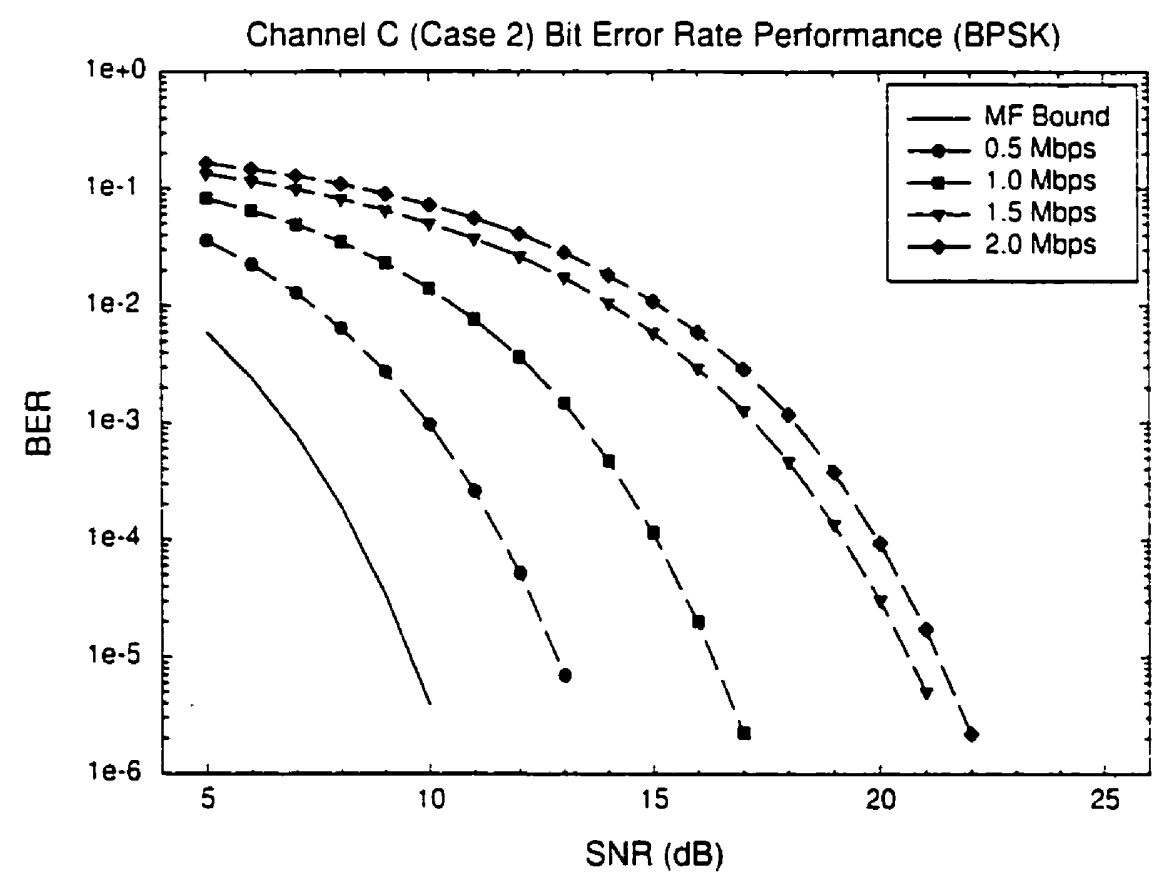

Figure 4.8: Channel C (Case 2) BER Results (BPSK) 


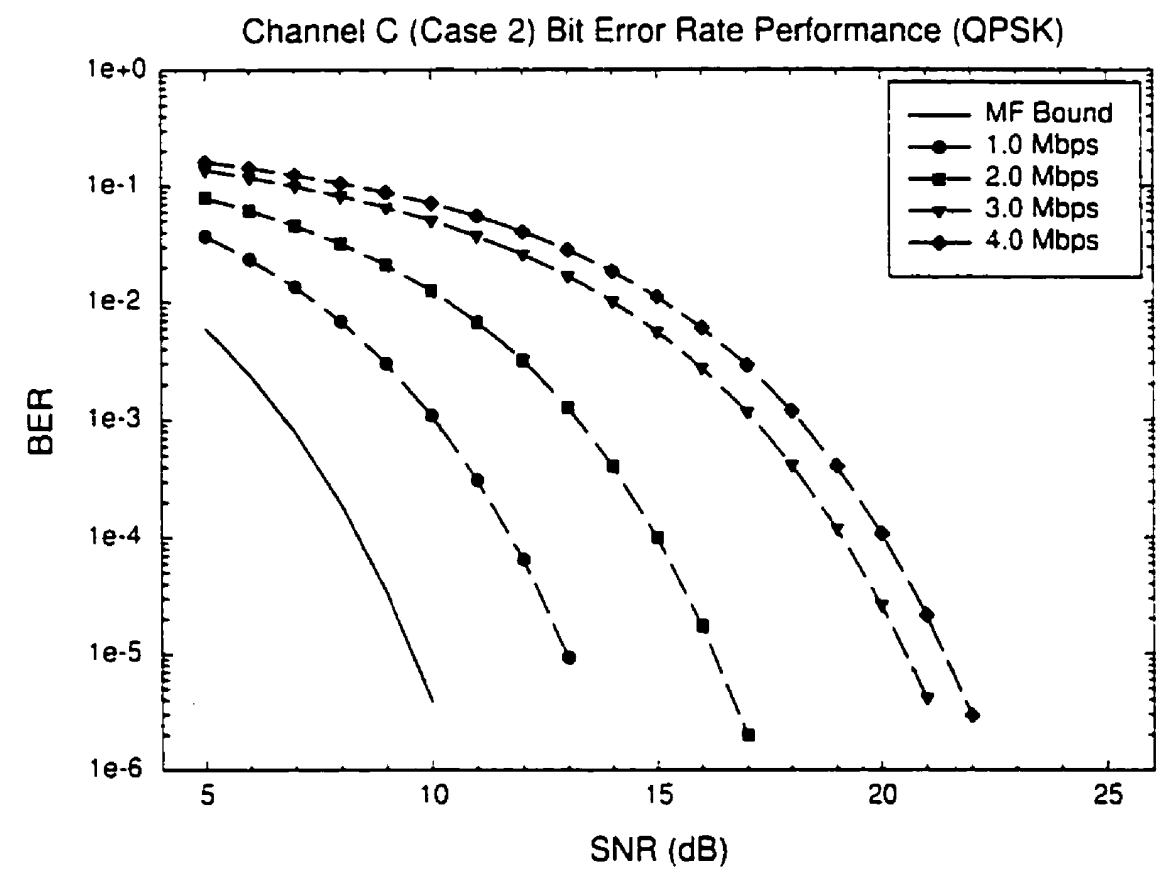

Figure 4.9: Channel C (Case 2) BER Results (QPSK) 


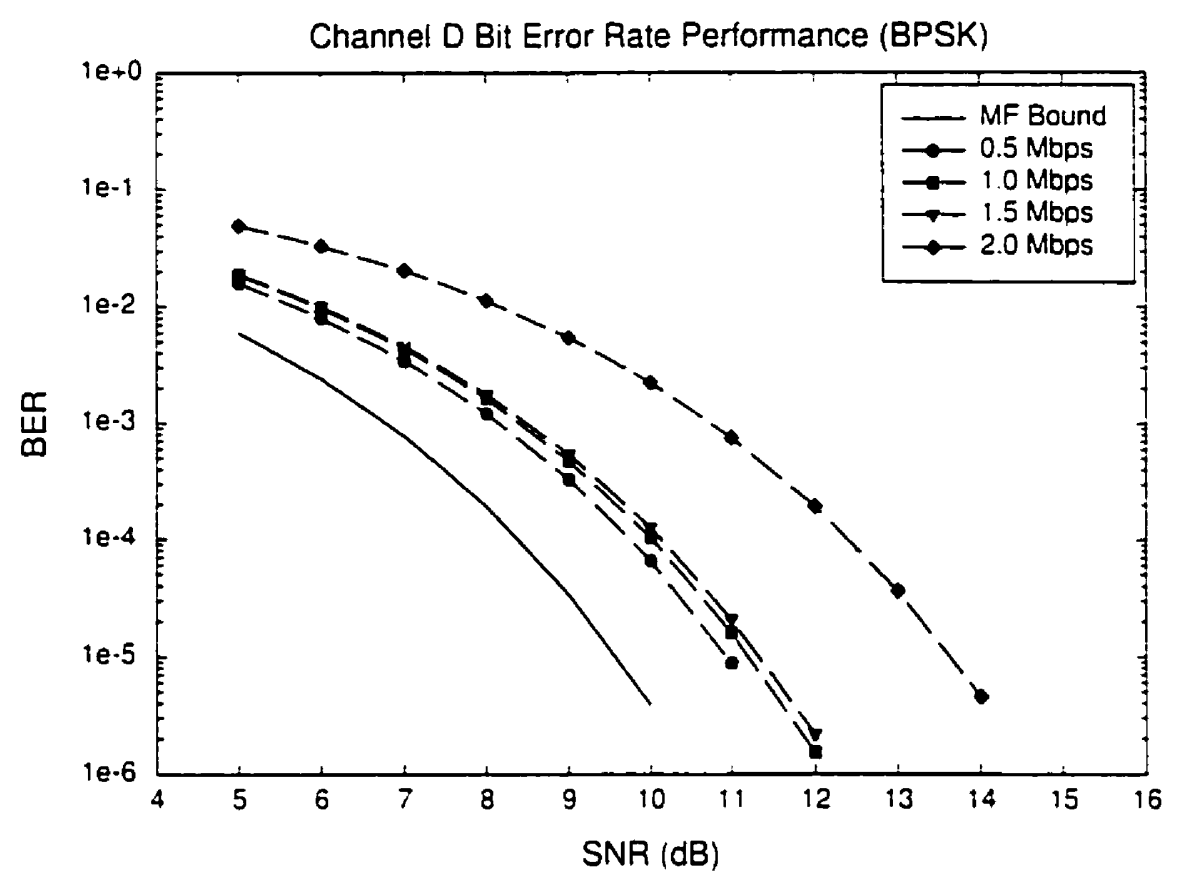

Figure 4.10: Channel D BER Results (BPSK)

significantly smaller than that of case 1.

Finally: the results for Channel $D$ are presented in Figures 4.10 and 4.11. They demonstrate that for Channel $D$ nearly the same performance can be achieved at data rates of 0.5. 1.0 and 1.5 Mbps for BPSK and 1.0. 2.0 and 3.0 . Mbps for QPSK modulation. Signal-to-noise ratio of $10 \mathrm{~dB}$ is sufficient to obtain BER of $10^{-4}$. In order to maintain the error rate of $10^{-4}$ at 2 and 4 IIbps respectively for BPSK and QPSK. an increase of $2.5 \mathrm{~dB}$ in signal power is necessary. 


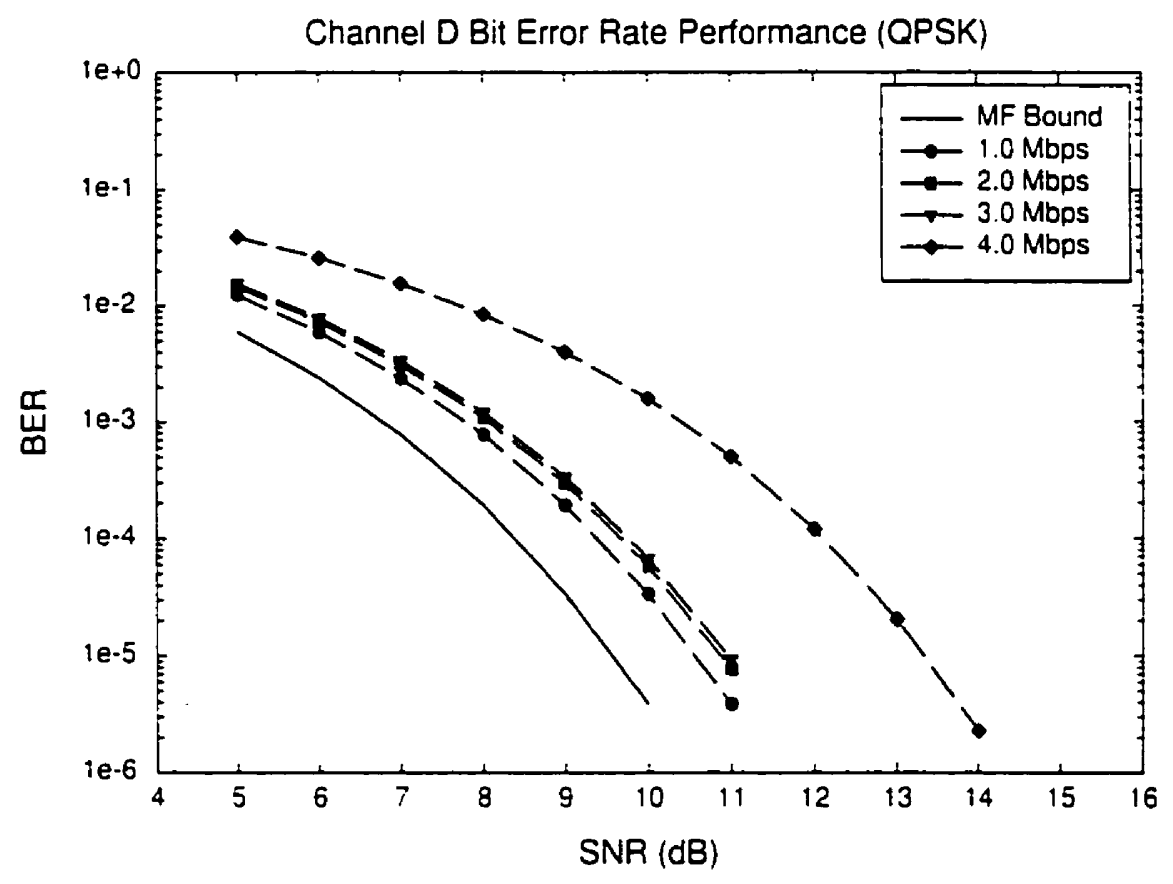

Figure 4.11: Channel D BER Results (QPSK) 


\subsubsection{BER Performance as a Function of Data Rate}

We now examine the relationship between the BER performance and the transmission rate. Shown in Figures 4.12 and 4.13 are the $B E R$ results (for $S . R=15 d B$ ) ats at function of the data rate. The following are the findings.

- BER performance for Channel $C$ is much poorer than that for Channel $D$. with a difference of approximately $T$ orders of magnitude at 1 Mbps BPSK (2 . Mbps (QPSK).

- After an initial spike. the BER for case 1 of Channel $C$ experiences a sudden drop at the data rate of 0.4 . Ibps BPSK (0.8 .Mbps QPSK). Similar drop occurs at 0.6 .lbps BPSK (1.2 Mbps QPSK) for case 2.

- Following the dip in BER. the performance for both cases of Channel C continues to degrade until 1.j .Mbps BPSK (3.0 .Mbps for QPSK). when the BER becomes steady at a non-practical value of $10^{-2}$.

- As expected from Figures 4.10 and 4.11. the BER over Channel D increases slowly with data rate between 0.5 and 1.5 Mrbps BPSK (1.0 and 3.0 Mbps QPSK). The performance degrades drastically at rates greater than 1.5 .lbps BPSK (3.0 .Mbps QPSK).

\subsubsection{Channel Distortion Analysis}

The large difference in performance between Channels $C$ and $D$ can be explained by examining the amount of intersymbol interference introduced by the channels. Figure 
High Rate Channel BER Performance (BPSK, SNR $=15 \mathrm{~dB}$ )

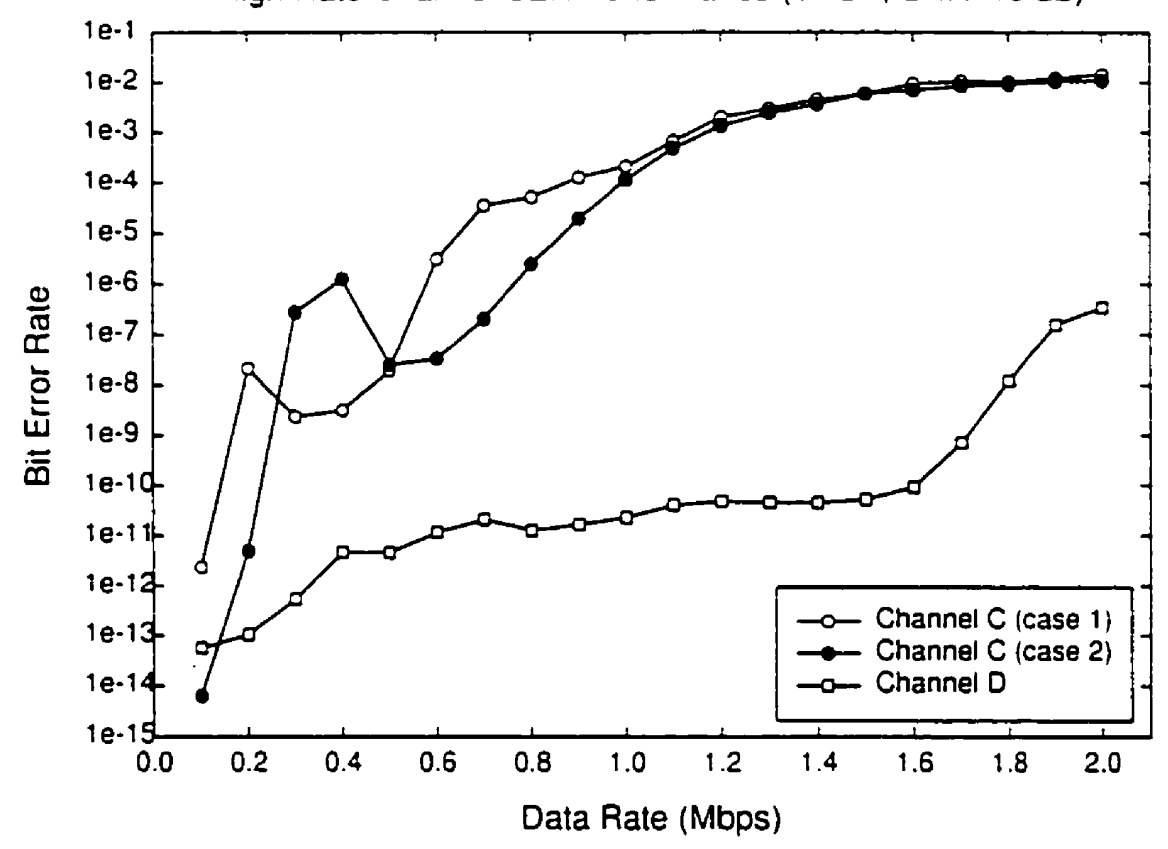

Figure 4.12: High Rate Channel BER as a Function of Data Rate (BPSK) 


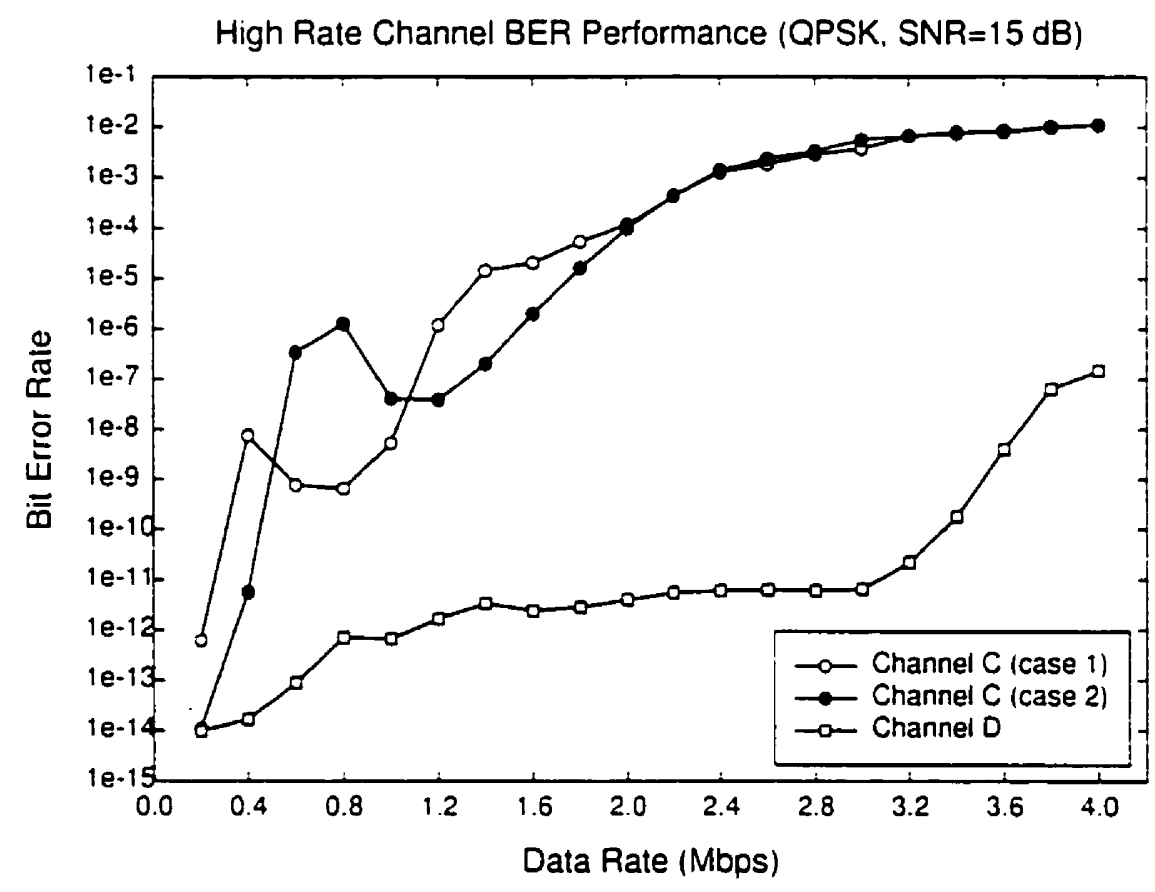

Figure 4.13: High Rate Channel BER as a Function of Data Rate (QPSK) 
4.14 shows the input signal-to-interference ratio (SIR). defined by

$$
S I R_{\text {unput }}=\frac{f_{0} f_{i}^{*}}{\sum_{i=0} f_{i} f_{i}^{*}} .
$$

where $f_{t}$ denotes the impulse response of the overall (unequalized) channel. To in(lude the contribution of noise. the input signal-to-noise-and-interference ratio (S.IR) defined by

$$
S . W R_{\text {tnput }}=\frac{f_{0} f_{i j}^{*}}{\sum_{i=0} f_{i} f_{i}^{*}-\sigma_{i}^{2}}
$$

where $\sigma_{r}^{2}$ denotes the total noise variance at the channel output. is shown in Figure 4.15. From Figures 4.14 and 4.15 it is clear that Channel $C$ introduces significantly more interference than Channel D. which can be expected since Channel $C$ has a longer delay spread. The difference in SIR and S.NIR predicts the large difference in BER performance.

In order to study the sudden drop in BER at 0.4 . Mbps for case 1 and 0.6 .lbps for case 2 of Channel C. we have also calculated the equalized S.IR. defined by

$$
S . Y^{\prime} I R_{\text {output }}=\frac{g_{01} y_{i j}^{*}}{\sum_{i \neq 0} y_{t} y_{i}^{*}+\sigma_{\Xi}^{2}} .
$$

where $\sigma_{:}^{2}$ denotes the total noise variance at the equalizer output. Output S:NIR is an indication of the equalizer's ability to combat the intersymbol interference introduced by the channel. Plots of SNIR as a function of data rate for BPSK and QPSK are shown in Figures 4.16 and 4.17 . The results show consistency with the bit error rate plots for all cases considered. The dip in BER for Channel $C$ at 0.4 Mbps BPSK is predicted by a sudden increase in the equalized SNIR. The relatively slow degradation in performance over Channel $\mathrm{D}$ with increasing data rate is also predicted by the shape 
High Rate Channel SIR

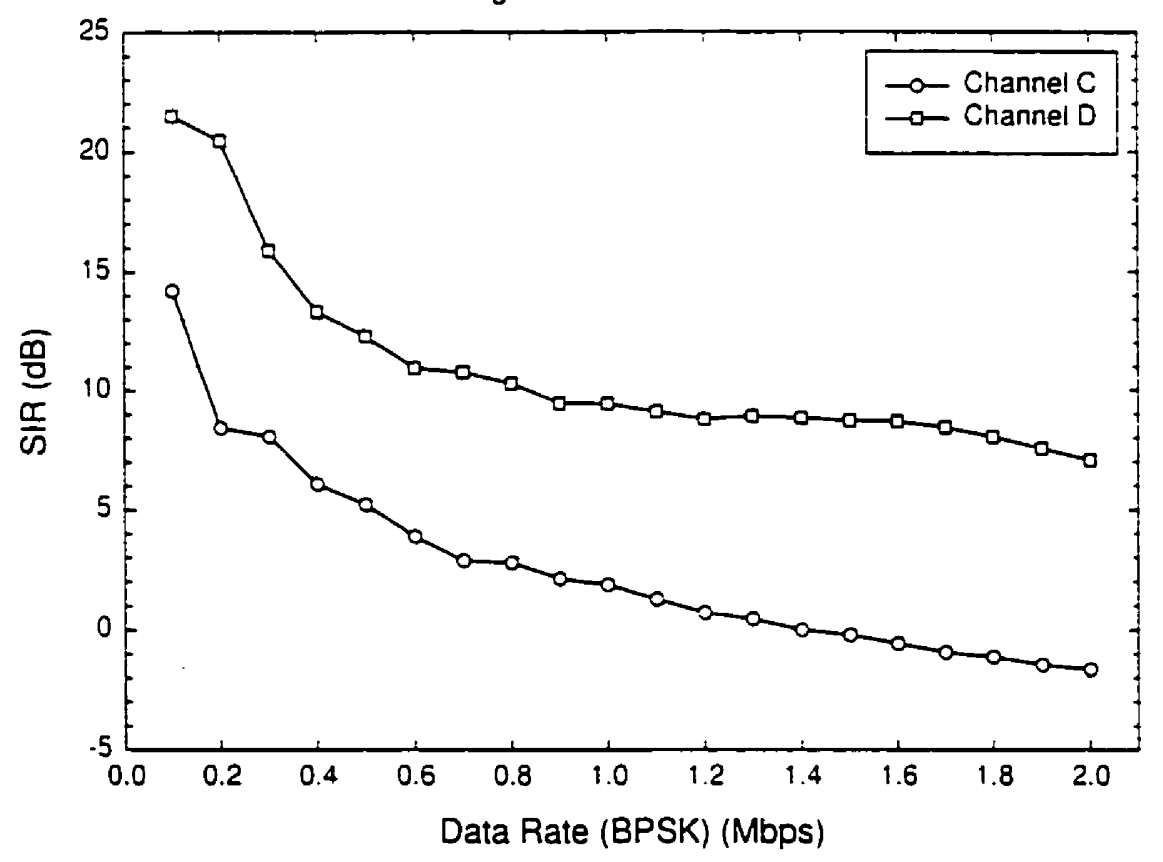

Figure 4.14: High Rate Channel Input SIR 
High Rate Channel Input SNIR

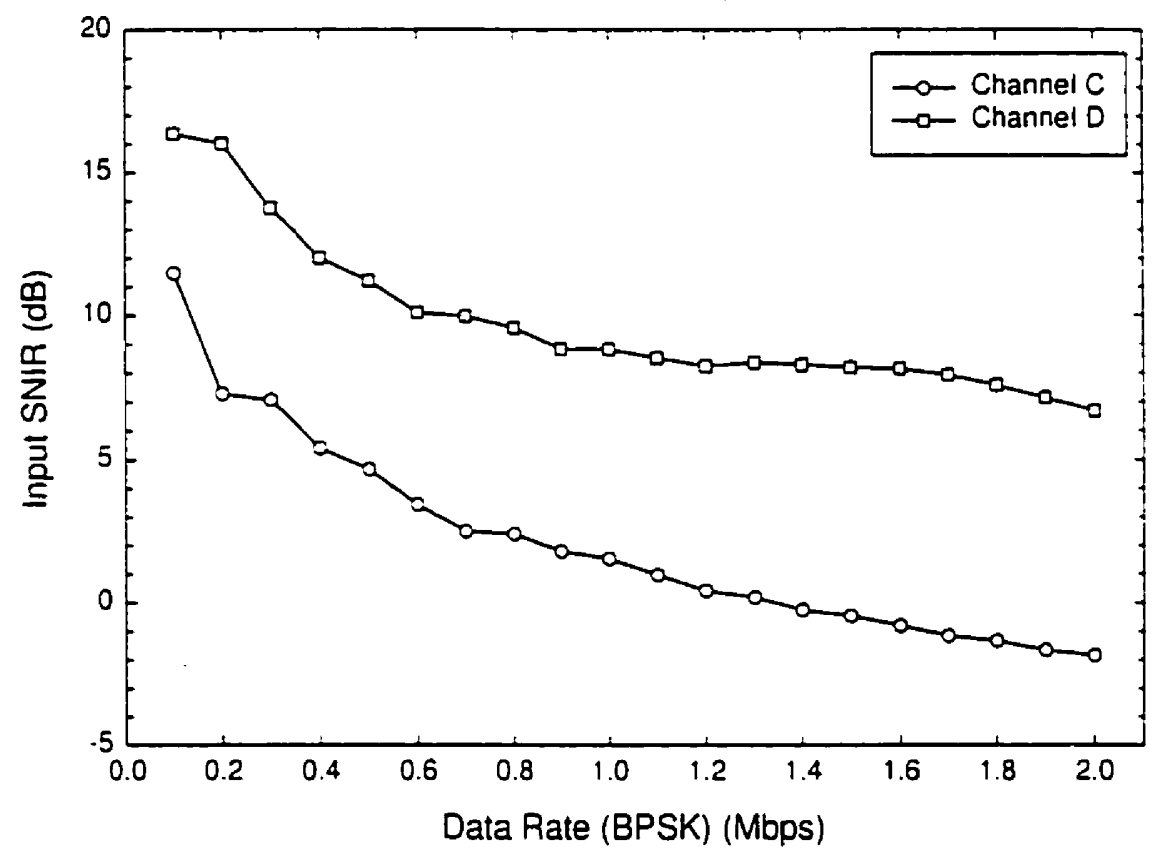

Figure 4.15: High Rate Channel Input SNIR 


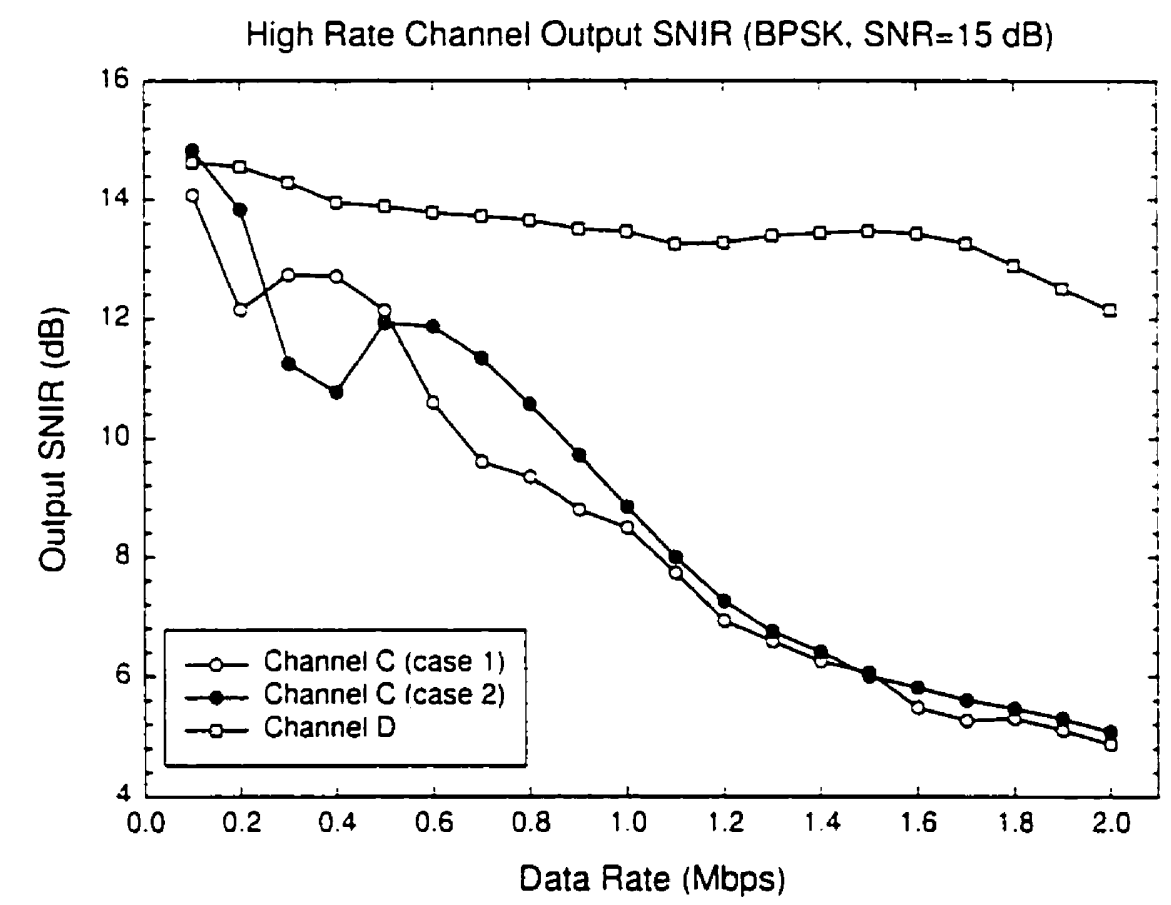

Figure 4.16: High Rate Channel ()utput S.IR (BPSK)

of the S.MIR curves which show a slow decrease in the amount of equalized distortion.

\subsection{Convergence Properties}

\subsubsection{Equalizer Learning Curves}

In this section we compare the convergence characteristics of the L.VS. FBL.IS and $\therefore F B L \lambda$ S algorithms for high data rate channels. The analysis is performed on Channel $C$ (case 1 ) and Channel $D$ at data rates of 2 Mbps BPSK and 4 . Mbps QPSK with S.NR fixed at $15 \mathrm{~dB}$. As required for an adequate bit error rate performance. the 
High Rate Channel Output SNIR (QPSK, SNR=15 dB)

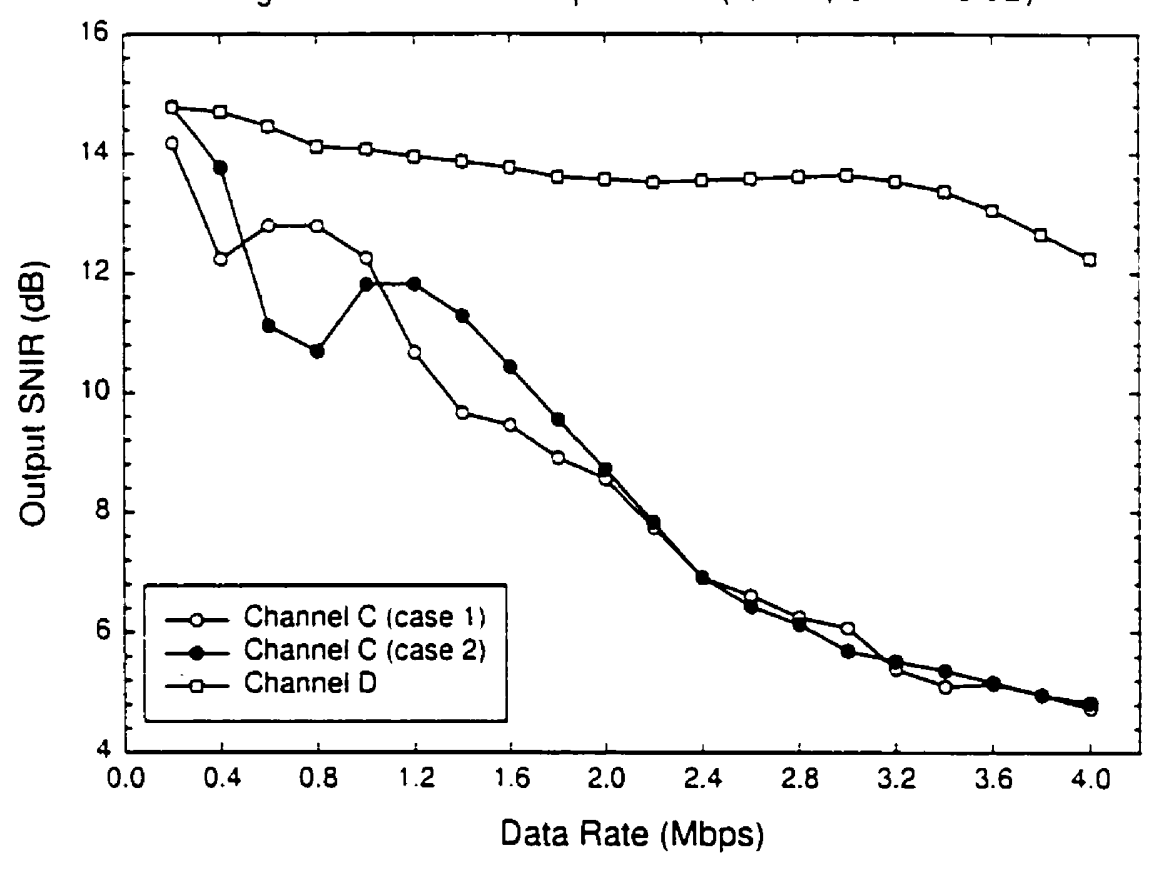

Figure 4.17: High Rate Channel Output SNIR (QPSK) 


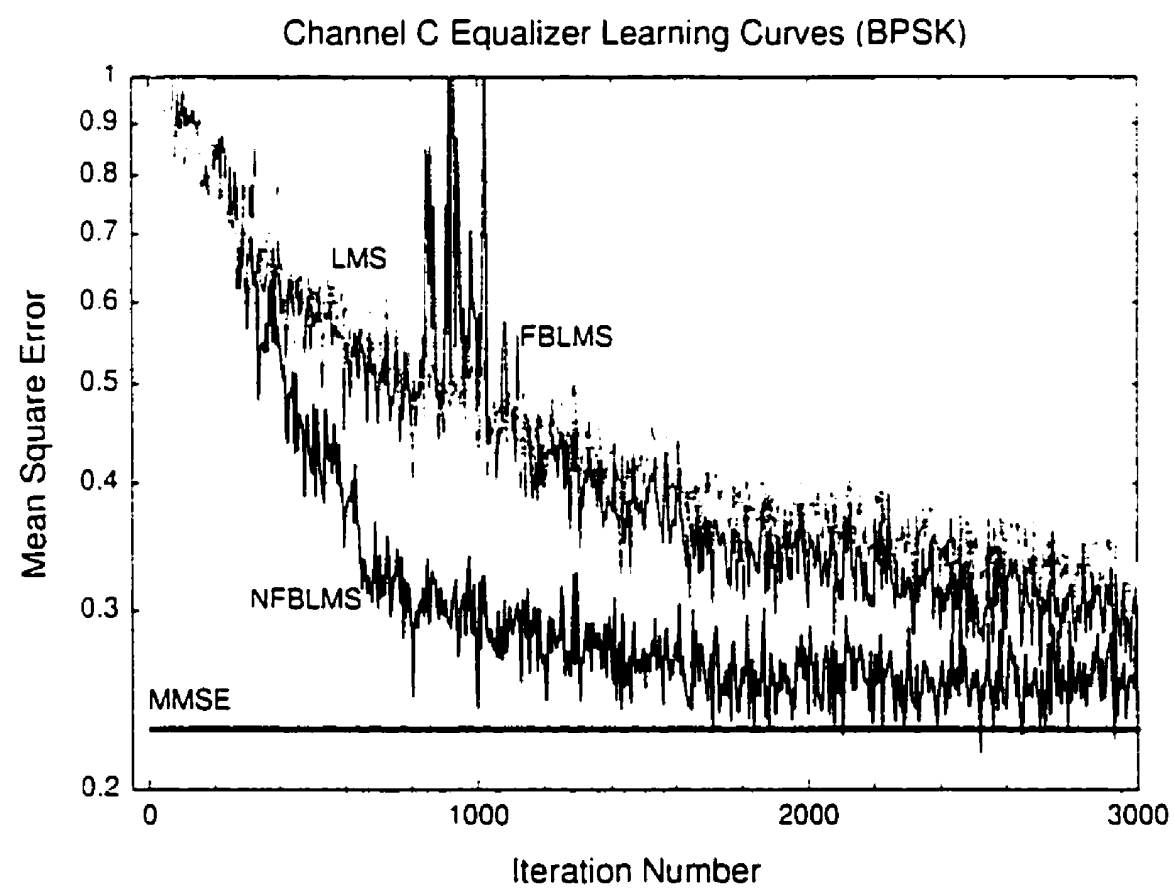

Figure 4.18: Channel C Equalizer Learning Curves (BPSK)

equalizer length at these data rates was set to 64 taps. The results were obtained by areraging an ensemble of 100 independent trials. In order to fairly compare the learning rharacteristics. individual curves in each graph were obtained using a step size chosen such as to result in the same steady state misadjustment. The step size was selected according to (2.26). (2.80) and (2.81) for a misadjustment value of $5 \%$.

Figures 4.18 and 4.19 show the learning curves of the various algorithms as applied to Channels $C$ and $D$ for BPSK modulation. Corresponding results for QPSK are plotted in Figures 4.20 and 4.21 .

As indicated by Figures 4.18 through 4.21 , the NFBL.IS algorithm offers a signif- 


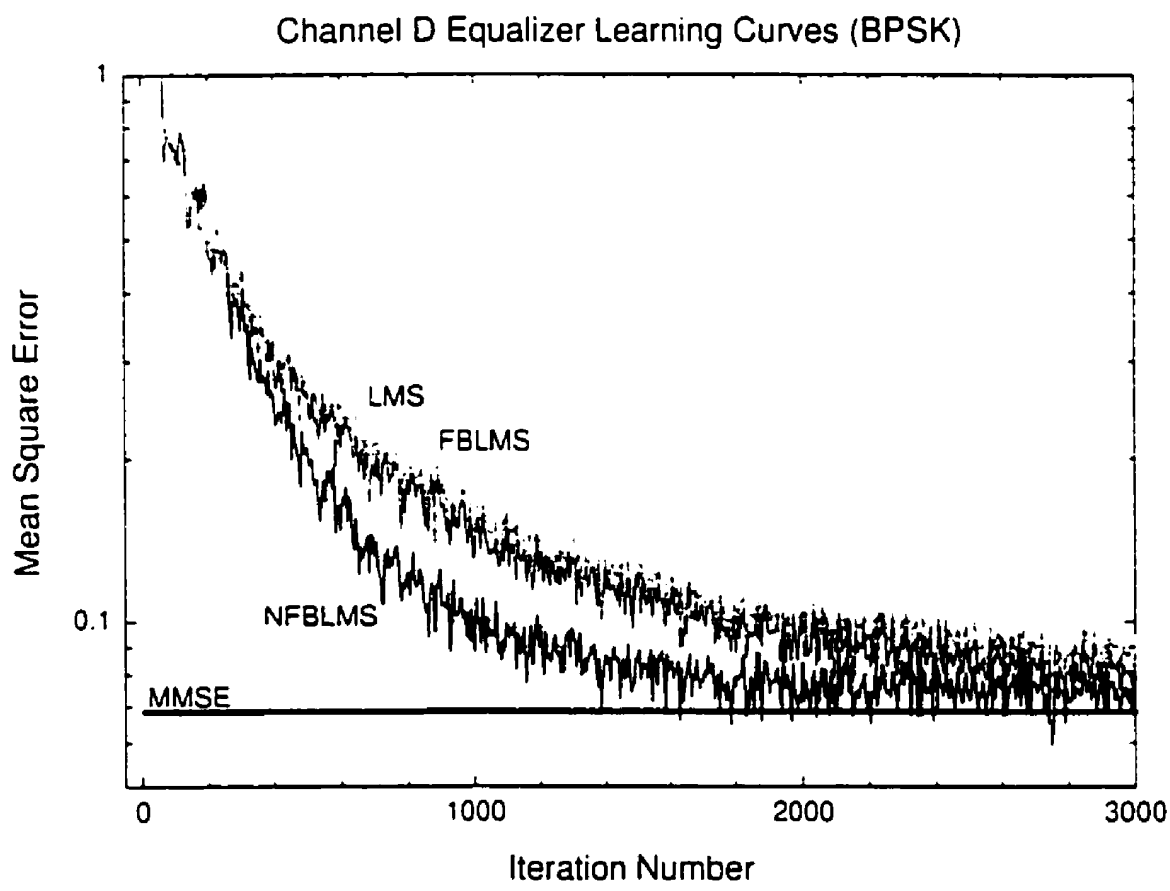

Figure 4.19: Channel D Equalizer Learning Curves (BPSK) 


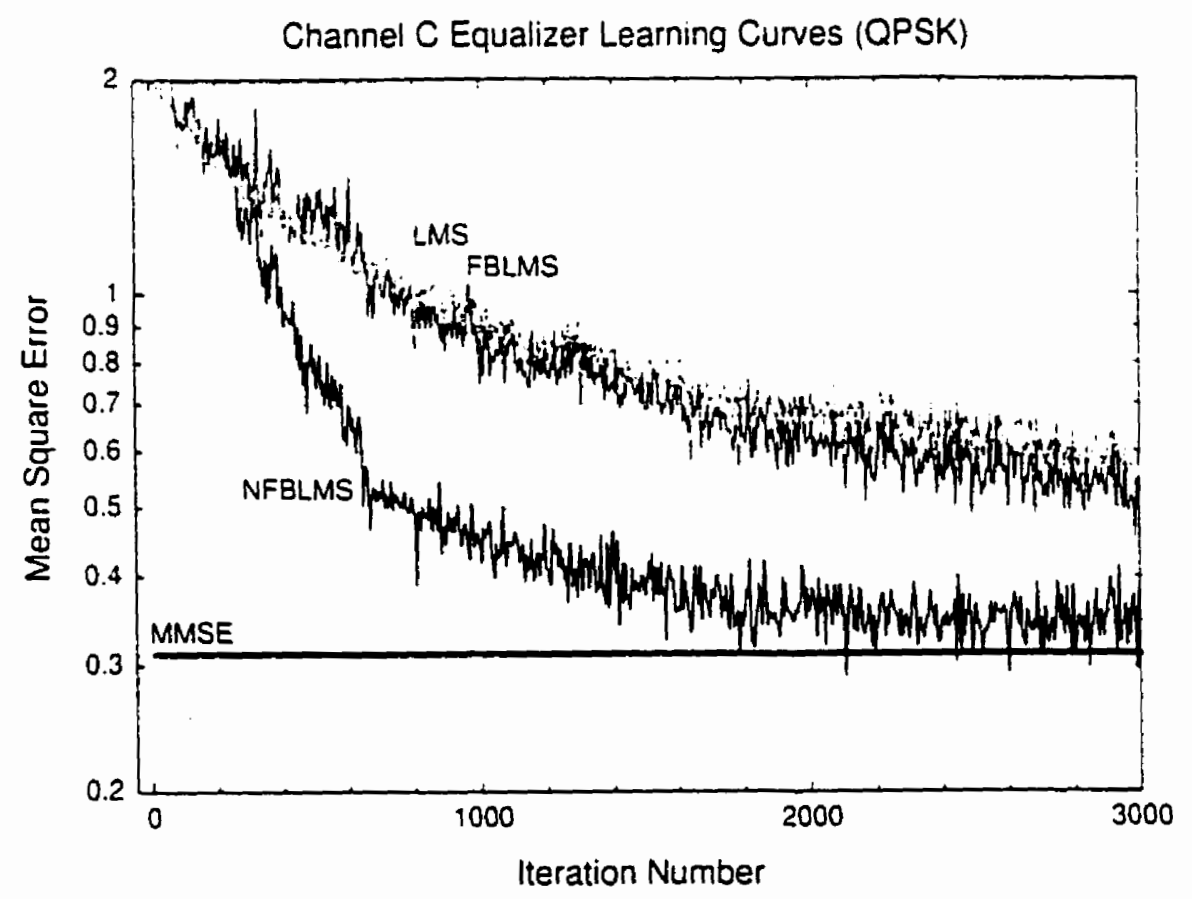

Figure 4.20: Channel C Equalizer Learning Curves (QPSK) 


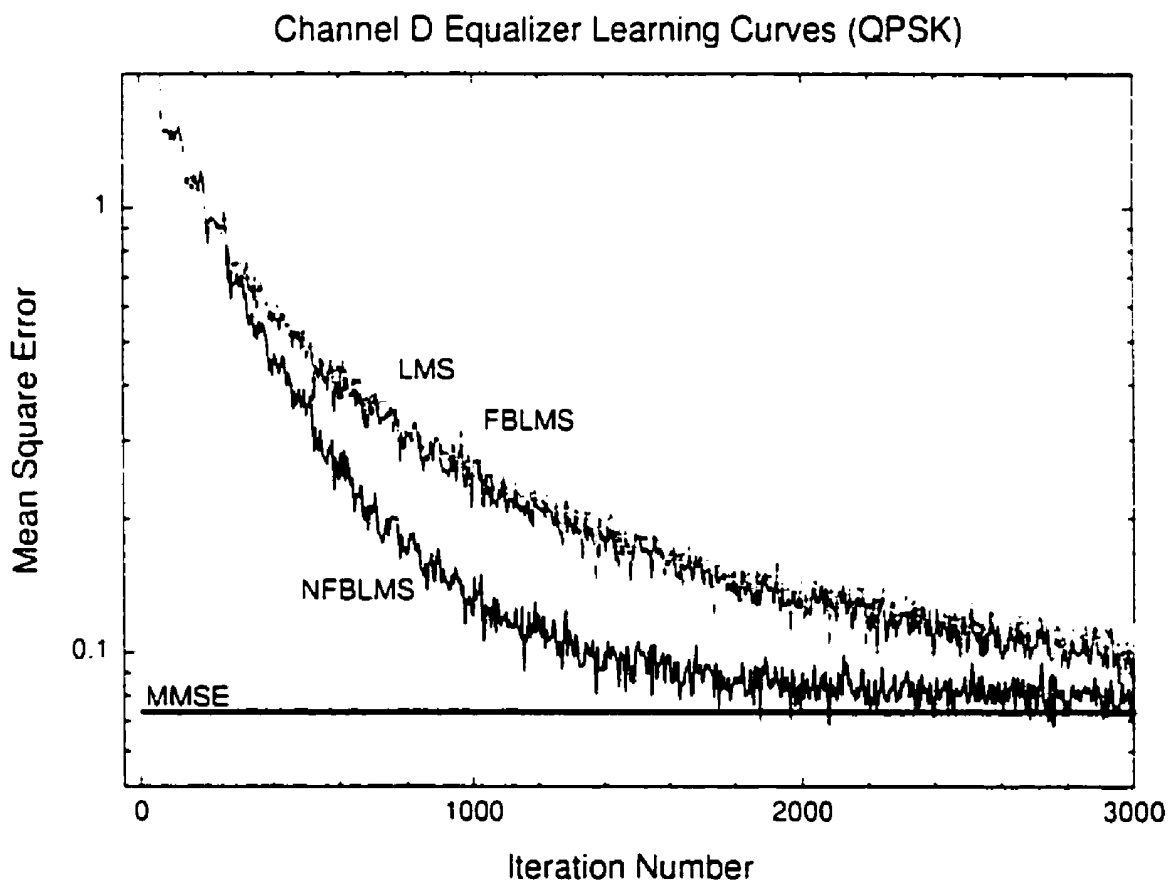

Figure 4.21: Channel D Equalizer Learning Curves (QPSK) 
Table 4.1: High Rate Channel Eigenvalue Spread Comparison

\begin{tabular}{|c|c|c|}
\hline$\frac{\lambda_{m .15}}{\lambda_{m . n}}$ & Channel C & Channel D \\
\hline L.IIS. FBL.ISS & 190.39 & 24.80 \\
\hline XFBL.IS & 12.02 & 4.01 \\
\hline
\end{tabular}

icant improvement in the speed of convergence. In all cases examined. the L.MS and FBL.IS algorithms required over 3000 iterations to reach the steady state solution. On the other hand. the .FBL.MS algorithm converged to the optimum tap values within 1.500 iterations. or approximately 25 blocks.

The fast convergence of the AFBL.MS algorithm also allowed for shorter training sequences. In all cases. stability was ensured with training sequences of only 10 blocks. The L.MS and FBL.MS algorithms required in excess of 50 training blocks in order to conterge to the optimum solution.

\subsubsection{Correlation Matrix Eigenvalue Analysis}

As stated in Chapter 2. the rates of convergence of the L.IS and NFBL.MS algorithms are governed by the ratio of the largest and smallest eigenvalues of their input correlation matrices. Table 4.1 presents a comparison of the eigenvalue disparity of the transformed and non-transformed correlation matrices. The results shown indicate that the eigenvalue spread reduction is greater in the case of Channel $C$. This is consistent with the learning curves presented in Figures 4.18 through 4.21 . which show that the improvement in the convergence is greater in the case of Channel $\mathrm{C}$.

As the tabulated results show, the Fuurier transform operation significantly reduces the eigenvalue disparity of the input process. In the cases examined. the eigenvalue spread is reduced by about an order of magnitude thus supporting the learning 
curve results previously presented.

The reduction of the eigentalue disparity for large .1 can be explained by close examination of the structure of the input correlation matrix. The following discussion is based on the work presented in [9]:31] and [3:?. Defining the auto-corrolation of the input $x_{n}$ as $\rho_{l}=E\left[x_{n}^{*} x_{n-l}\right]$ for $\left.l=0.1 \ldots \ldots\right)-1$. we have the alute-correlation matrix

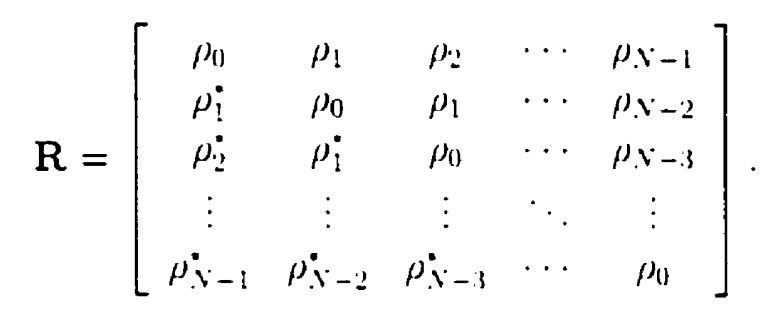

From $(4.4)$ we see that the correlation matrix $R$ is Toeplitz and Hernitian. It can be shown :33! that when $\rho_{l}$ approaches zero for lag values $l$ greater then $m$ ( $m$ much smaller than $. \mathrm{V} / 2$ ). the eigenvalue distribution of $\mathrm{R}$ is asymptotically equivalent to the eigentulue distribution of a circular matrix $\mathrm{C}$. defined as

$$
\mathbf{C}=\left[\begin{array}{cccccccccc}
\rho_{0} & \rho_{1} & \cdots & \rho_{m} & & & \rho_{m}^{*} & \cdots & \rho_{2}^{*} & \rho_{i}^{*} \\
\rho_{\mathrm{i}} & \rho_{0} & \ddots & \vdots & \rho_{m} & & & \ddots & & \rho_{i}^{;} \\
\vdots & \rho_{\mathrm{i}}^{*} & \ddots & \rho_{1} & \vdots & \ddots & & 0 & \rho_{m}^{*} & \vdots \\
\rho_{m}^{*} & \vdots & \ddots & \rho_{0} & \rho_{1} & & \rho_{m} & & & \rho_{m}^{*} \\
& \rho_{m}^{*} & & \rho_{\mathrm{i}} & \rho_{0} & \ddots & \vdots & \ddots & & \\
& & \ddots & \vdots & \rho_{\mathrm{i}}^{*} & \ddots & \rho_{\mathrm{l}} & & \ddots & \\
\rho_{m} & & & \rho_{m}^{*} & \vdots & \ddots & \rho_{0} & \ddots & & \rho_{m} \\
\vdots & \ddots & 0 & & \rho_{m}^{*} & & \rho_{\mathrm{i}}^{*} & \ddots & \ddots & \vdots \\
\rho_{2} & & \rho_{m} & & & \ddots & \vdots & \ddots & \rho_{0} & \rho_{1} \\
\rho_{1} & \rho_{2} & \cdots & \rho_{m} & & & \rho_{m}^{*} & \cdots & \rho_{1}^{*} & \rho_{0}
\end{array}\right] .
$$

The circular matrix $\mathbf{C}$ is obtained from $\mathbf{R}$ by appropriately filling in its upper-right and lower-left corners. When the correlation matrix $\mathrm{R}$ is approximately circular. corresponding frequency domain correlation matrix $\mathbf{R}_{\mathcal{F}}$ is approximately diagonal 
[1.j]

$$
\begin{aligned}
\mathrm{R}_{F} & =\mathcal{F} \mathrm{C} \mathcal{F}^{H} \\
& \left.=\text { Nif } \mathcal{F C F}^{-1}\right) \\
& =\text { Nidiagir } ; .
\end{aligned}
$$

where $r_{\mathcal{F}}=\mathcal{F} r$. that is. the Fourier transform of the first column of $\mathrm{R}$. This shows that when the decaly time of the input auto-correlation is small compared to filter length.. . the Fourier-transformed correlation matrix is approximately diagonal. As described in Section 2.4. proper step normalization is equivalent to obtaining a correlation matrix close to identity: which in turn has a small eigenvalue spread.

\subsection{Computational Complexity}

Wi" now compare the computational complexity of the L.IS. FBL.IS and NFBL.IS equalizers applied to the high data rate channels. Since all cases considered thus far in this chapter included a receive filter of constant complexity: only the number of real multiplications to process a block of data of length.$V^{\prime}$ at the equalizers is included in the calculations.

Table 4.2 shows the total number of multiplications required to process a block of data of length.$Y$ using the L.VS. FBL.SS and NFBL.MS algorithms for real input sequence (BPSK modulation). As discussed in Chapter 2. the total number of real multiplications used by the L.ISS. FBL.MS and NFBL.MS algorithms for a block of . symbols are $2 \mathrm{~V}^{2} .10 . \mathrm{Vog} 2 \mathrm{~N}+16 . \mathrm{V}$ and $10 \mathrm{~V} \log 2 \mathrm{~N}+20 . \mathrm{V}$ respectively. For data rates less than 1 Mbps (BPSh). equalizers of length.$=32$ were used. with $N$ increasing to 64 . for rates of 1 to $2 \mathrm{Mbps}$ (BPSK). We see that for equalizer length of 64 both the non-normalized and normalized frequency domain algorithms offer computational 
Table 4.2: Equalizer Complexity for High Rate Channels (BPSK)

\begin{tabular}{|c|c|c|}
\hline & $\therefore=32$ & $\therefore=64$ \\
\hline L.IS & 2048 & 8192 \\
\hline FBL.ISS & 2432 & 5.504 \\
\hline IFBL.ISS & 2560 & .5760 \\
\hline
\end{tabular}

savings over the time clomain implementation.

For QPSK signaling. the total number of real multiplications used br the LMS. FBL.IS and .NFBL.IS algorithms for a block of $.1^{\circ}$ symbols are $8.1^{2} .20 .1 \% \log 2.1^{\circ}+16.1^{\circ}$ and $20.1 \% \log 2.1^{\circ}+20.1^{\circ}$ respectively: In the (ase QPSK modulation. where the input data is complex. the reduction in complexity is noticeable for all cases examined here. The results are tabulated in Table 4.3. The increase in the computational savings for

Table 4.3: Equalizer Complexity for High Rate Channels (QPSK)

\begin{tabular}{|c|c|c|}
\hline &.$=32$ &. \\
\hline L.IS & 8192 & 32768 \\
\hline FBL.IS & 4352 & 9984 \\
\hline IFBL.IS & 4480 & 10240 \\
\hline
\end{tabular}

complex input data is due to the fact that the time domain implementation requires 4 times the number multiplications compared to real data. The frequency domain algorithm. which operate with complex numbers regardless of the type of input. only have a two-fold increase in computations resulting from the loss of symmetry of the FFT algorithm. Thus, the frequency domain implementations offer savings of $45 \%$. and $69 \%$ for filter lengths of.$=32$ and.$=64$.

As stated in [14]. many DSP's are capable of performing up to $1.6 \times 10^{9}$ real operations per second. Assuming a transmission rate of $2 \times 10^{6}$ complex symbols per 
secund. the DSP will be capable of performing approximately 800 real operations per srmbol. Taking into account that the values given in Tables 4.2 and 4.3 are for real operations for blocks of 32 and 64 symbols. it can be seen that. at the above data rate. modern DSP's should be well suitable for equalization applications. 


\section{Chapter 5}

\section{Conclusion}

\subsection{Conclusions}

This thesis presented a comparison of time domain and frequency domain equalizers. Specitically, we examined the BER performance. cunvergence characteristics and computational complexity of L.MS. FBL.IS and NFBL.IS algorithms as applied to static multipath channels with short and long delay spreads. The conclusions of this study are as follows:

1. The block L.IS algorithm can be efficiently implemented in the frequency domain using the overlap-save data sectioning and a gradient constraint. The resulting FBL.MS and NFBLAS algorithms converge to the same sets of equalizer taps as their LISS counterpart. The bit error rate performance of all of these equalizers is thus identical.

2. A fractionally-spaced FBL.MS and NFBL.MS equalizers have the capability of performing the function of a receive filter. In cases when the span of the receive filter is approximately equal to that of the equalizer. the same BER performance can be obtained by eliminating the former and increasing the sampling rate of the equalizer. Due to the low complexity growth with an increase of the number 
of taps. the computational complexity of the fractionally-spaced equalizer is romparable to the combined complexity of the receite filter and a stmbol-spaced equalizer.

3. The BER performance was studied for two high data rate multipath channels with random phase characteristics. The first channel. with maximum delay spread of $8 \mu s$. was examined for two different phase profiles. In the first case. for which the channel spectrum contained an inband null. a signal-t(o-noise ratio of 11.j $\mathrm{dB}$ was required to achieve a BER of $10^{-4}$ at data rate of 0.5 Mhps BPSK (1.0 Mbps QPSK). Such performance can be maintained with an increase in S.TR of $4 \mathrm{~dB}$ for each increase in data rate of 0.5 Mbps BPSK until $1.5 \mathrm{Mbps}$. Prformance drop between 1.j and 2.0 .Mbps BPSK (3.0 and 4.0 Mbps QPSK) Was approximately 1.5 dB. The second case of channel one. one without an inband null. resulted in similar BER performance. The lack of performance improvement for case 2 was attributed to the smaller channel bandwidth. The second high data rate channel studied. one with a maximum delay spread of $5 \mu$ s. required an S.IR of approximately $10 \mathrm{~dB}$ to achieve BER of $10^{-4}$ for the rates of 0.5 to $1.5 \mathrm{BPSK}$ and 1.0 to $3.0 \mathrm{MHps}$ QPSK. In order to increase the data rate to 2.0 and 4.0 .Mbps. a S.VR increase of $2.5 \mathrm{~dB}$ was needed.

4. By varying the step size in the adaptation process according to the power distribution of the input signal. the resulting NFBL.MS algorithm exhibits a much greater convergence rate than the L.IS and FBL.MS implementations. The improvement is most noticeable for long equalizers where the input data correlation matrix is approximately circular and thus diagonalized by the Fourier transform. When an appropriate normalization technique is implemented. the 
eigenvalue disparity is greatly reduced. resulting in a uniform convergence across all modes. As a result. for both high data rate channels examined. the .VFBL.MS algorithm converged to a steady state after about 25 data blocks. In all of the rases examined the L.IS and FBL.MS algorithms failed ro converge after pro('essing jo data blocks.

j. For long equalizer responses. the XFBL.MS and FBL.MS algorithms offer signiticant savings in computational complexity over the L.IS counterpart. For BPSK signaling. the frequency domain implementations vield an improvement of $3.5 \%$ for block lengths of 64 . In the case of QPSK transmission. gains of $50 \%$. $65 \%$ are obrained for block lengths of 32 and 64 .

\subsection{Suggestions for Further Research}

The frequency domain block algorithms offer significant computational savings for long equalizers. The algorithm studied in this thesis is based on a constrained gradient approach. The advantage of this adaptation technique is that the steady state equalizer coefficients are equal to the 11 iener solution. The gradient constraint. however. can be omitted in favor of eliminating two of the FFT operations. It has been shown by Mansour and Gray [34] that when the filter length is sufficiently large. such an algorithm will converge to a similar tap contiguration as the constrained counterpart. As pointed out by [13] and [15], while this algorithm is much simpler to implement. it does come at a cost of a higher misadjustment for a given step size. Despite the above shortcoming. it may be worthwhile to examine the performance of the unconstrained FBL.MS equalizer for high data rate channels. Apart from reduced computational load. the convergence rate should also be improved over the L.MS algorithm since. as shown by [31]. the frequency domain correlation matrix of such an 
algorithm is also approximately diagonal.

One of the traits of fractionally-spaced equalizers is their ability tu compensare for a poor choice of timing phase at the receiver 35.5 . While in this thesis we hate in all (ases optimized the timing phase. it would be of interest to examine the ability of the fractionally-spaced frequency domain equalizers to compensate for delay distortion introduced by the system.

While the convergence rate of the MFBL.MS equalizers was shown to be much better than that of the L.US and FBL.IS implementations. it still remains on the order of 2.5 data blocks in the cases of long equalizer responses. Af further improrement in the learning curve can be obtained by implementing a frequency domain RLS algorithm. as these types of equalizers are usually superior in their convergence properties in comparison to the L.MS equalizer [18].

In examining high data rate channels. we have limited ourselves to static impulse responses. Consequently, tracking properties of the frequency domain equalizers were not analyzed. One may perhaps wish to analyze the combined effects of the delay introduced by block processing. as well as the relatively fast rate of convergence. on the ability of the frequency domain equalizer to track temporal variations in the channel impulse response.

Finally: it is known [18] that the linear equalizer suffers great performance degradation in cases in which the involved channel exhibits bad spectral characteristics (deep spectral nulls). The most common solution to this problem is the Decision Feedback Equalization (DFE). In the recent literature. several authors have worked on developing frequency implementations of block decision feedback equalizers [36] [3i]. It may be worthwhile to examine the performance of the developed structures as applied to the high data rate wireless environment. 


\section{Bibliography}

1': R. II. Lucky: Automatic equalization for digital communications. Bell System Technecal Journal. 44:54-5-588. April 1965.

2) R. IV. Lucky. Techniques for adaptive equalization of digital communications systems. Bell System Terhnucal Journal. 45(2):255-286. February 1966.

[3] B. Widrow and M. E. Hoff Jr. Adaptive switching circuits. IRE WESCON Concention Recort. pages 563-583. 1960.

[4] B. Widrow. Adaptive filters I: Fundamentals. Stanford Electronic:s Laboratory. Stanford. CA. Tech Report No. 6i6t-6. December 1966.

[5] T. Waltzman and M. Schwartz. Automatic equalization using the discrete frequency domain. IEEE Transactions on Information Theory. IT-19(1):59-68. January 1973.

[6] M. Dentino. J. McCool. and B. Widrow. Adaptive filtering in the frequency domain. Proceedings of the IEEE. 66(12):1658-1659. December 1978.

[i] E. R. Ferrara. Fast implementation of L.IS adaptive filters. IEEE Transactions on Acoustics. Speech, and Signal Processing. ASSP-28(4):4it-475. August 1980.

[8] S. S. Naravan and A. M. Peterson. Frequency domain least-mean-square algorithm. Proceedings of the IEEE. 69(1):124-126. January 1981. 
i! J. C. Lee and C. K. Lu. Performance of transform-domain L.IS adaptive algorithms. IEEE Transactzons on Acoustes. Speech. and Signal Processining. ASSP$34: 499-510.1986$.

[10] D. F. Marshall. W. K. Jenkins. and J. J. Murphy: The use of orthogonal transforms for improving performance of adaptive tilters. IEEE Transurtuons on Circutts and Systems. CS-36(4):4it-483. April 1989.

111. B. Farhang-Boroujeny and S. Cazor. Selection of orthonormal transforms for improving the performance of the transform domain normalised L.IS algorithm. IEE Proceeding.s-F. 139(5):1992. October 1992.

[12] B. Widrow and S. D. Stearns. Adaptive Signal Processing. Prentice-Hall. Englewood Cliffs. N.J. 1985.

[13] J. J. Shynk. Frequency-domain and multirate adaptive filtering. IEEE Signal Processing Magazine. 9(1):14*37. January 1992.

[14] M. V. Clark. Adaptive frequency-domain equalization and diversity combining for broadband wireless communications. IEEE Journal on Selected Arcas in Communications. 16(8):1385-1395. October 1998.

[15] B. Farhang-Boroujeny: Adaptive Filters: Theory and Applications. John Wiley \&. Sons Ltd.. Toronto. ON. 1998.

[16] S. Havkin. Adaptive Filter Theory. Prentice Hall. Lpper Saddle River. L.J. 1996.

[17] J. G. Proakis and D. G. Manolakis. Digital Signal Processing. Principles. Algorithm. and Applications. Prentice-Hall. Toronto. ON. 1996.

[18] J. G. Proakis. Digital Communications. McGraw-Hill. New York. NY. 1995. 
[19! A. Papoulis. Probability. Random Lariables, and Stochastic Processeses. MrcGrawHill. Toronto. Oハ. 1991.

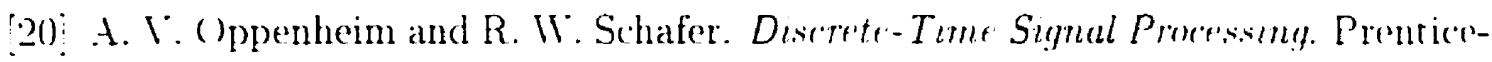
Hall. Englewood Cliffs. N.J. 1989.

21 J. II. Cooley et al. The fast Fourier transform algorithm: Programming considerations in the calculation of sine. cosine and Laplace transforms. Iournul of Sound and Vibratoons. 12:315-337. July 1970.

1.2.2) J. E. Smeer. Error-rate evaluation of lineatr equalization and decision feedback equalization. Master's thesis. Queen's L'niversity. 1995.

23] T. S. Rappaport. Wireless. Communucatoons. Theory and Practuce. Prentice Hall. Lpper Saddle River. … 1996.

[24] E. A. Lee and D. G. Messerschmitt. Dightal Communication. Kluwer Academic Publishers. Norwell. MA. 1994.

[25] J. F. Haves R. D. Gitlin and S. B. Weinstein. Data Communications Principles. Plenum Press. New York. NY. 1992.

[26] ․ C. Beaulieu. The eraluation of error probabilities for intersymbol and cochannel interference. IEEE Transactions on Communications. 39(12):1740-1749. December 1991.

[2i] J. E. Smee and X. C. Beaulieu. Error-rate evaluation of linear equalization and decision feedback equalization with error propagation. IEEE Transactions on Communications. 46(5):656-664. . Lay 1998. 
28: G. L. Stuber. Principles of Mobile Communcatıon. Kluwer Academic Publishers. Norwell. M.A. 1996.

29: T. J. Hicks. Time spread characterization of outdoor microcellular and indoor wireless $1.9 \mathrm{GHz}$ radio channels. Master's thesis. Queen S Lniversity. 199-

30) L. IV. Couch II. Degital and Analog Communication Systems. Macmillan Publishing Co.. Inc.. New York. .1: 1983.

[31: J. C. Lee and C. K. Lin. Performance analysis of frequency-domain block L.IS adlaptive digital filters. IEEE Transactzons on Circuits and Systems.s. 36(2):173189. February 1989.

[32! B. Farhang-Boroujeny and $\mathrm{k}$. S. Chan. Analysis of the frequency-domain block L.MS algorithm. IEEE Transactions on Signal Processing. 48(8):2000). August. 2000 .

[33] R. .I. Gray: On the asymptotic eigencalue distribution of Toeplitz matrices. IEEE Transactions on Information Theory. IT-18:72j-i30. November 1972.

[3-3] D. Mansour and A. H. Gray Jr. Lnconstrained frequency domain adaptive filter. IEEE Transactions on Acoustics. Speech. and Signal Processing. ASSP-30:726734. October 1982.

[35] R. D. Gitlin and S. B. Weinstein. Fractionally-spaced equalization: An improved digital transversal equalizer. The Bell Technical Journal. 60(2):275-298. February 1981. 
[36] $K$. Berberidis and J. Palicot. At frequency-domain decision feedback equalizer for multipath echo cancellation. Procededng!s from 1995 IEEE GLOBECOMM Conference. Nowember 199.j.

37 K. Berberidis and J. Palicot. Efficient block implementation of the decision feredback equalizer. IEEE Siqnal Processing Letters. j(6):1998. June 1998. 